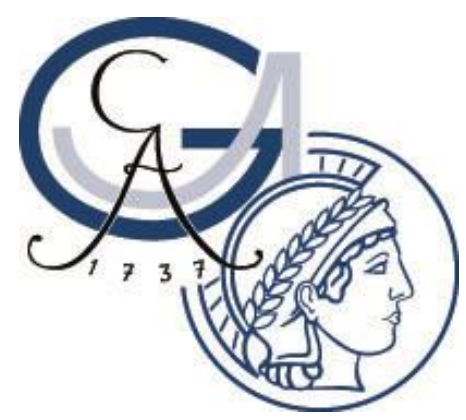

\title{
Microglia activation and regulation of remyelination in the central nervous system
}

\author{
Dissertation \\ for the award of the degree \\ "Doctor of Philosophy" \\ Division of Mathematics and Natural Sciences \\ of the Georg-August-Universität Göttingen \\ within the doctoral program Molecular Biology \\ of the Georg-August University School of Science (GAUSS) \\ submitted by \\ Minhui Su \\ from Shanghai, China \\ Göttingen 2018
}




\section{Members of the thesis advisory committee}

Prof. Dr. Mikael Simons (Reviewer)

Institute of Neuronal Cell Biology

Technical University of Munich

German Center for Neurodegenerative Diseases (DZNE)

Formerly: Max Planck Institute for Experimental Medicine

Prof. Dr. Blanche Schwappach (Reviewer)

Department of Molecular Biology

Institute of Molecular Biology

University Medical Center Göttingen

Prof. Dr. Steven Johnsen

Professor for Translational Cancer Research

University Medical Center Göttingen

\section{Additional members of the examination board}

Prof. Dr. André Fischer

Dept. for Psychiatry and Psychotherapy

University Medical Center Göttingen

German Center for Neurodegenerative Diseases (DZNE)

Prof. Dr. Dr. Hannelore Ehrenreich

Clinical Neuroscience

Max Planck Institute for Experimental Medicine

Prof. Dr. Tiago Fleming Outeiro

Department of Experimental Neurodegeneration

University Medical Center Göttingen

Date of oral examination: 27 November 2018 


\section{Table of Contents}

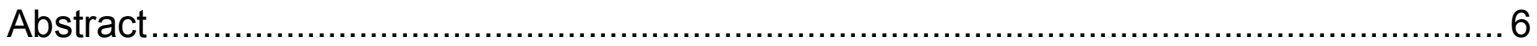

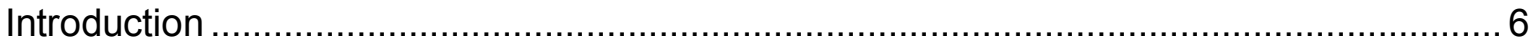

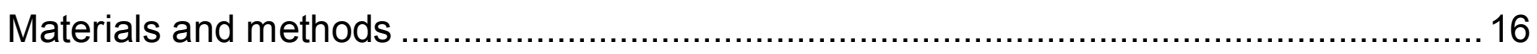

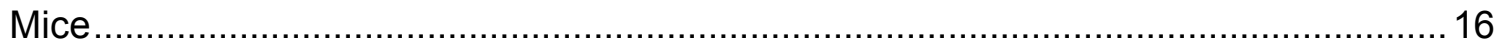

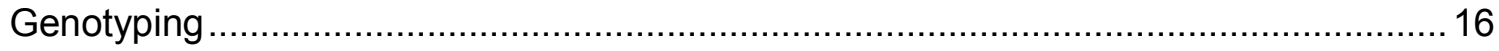

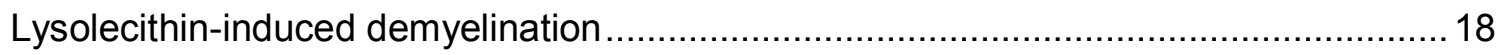

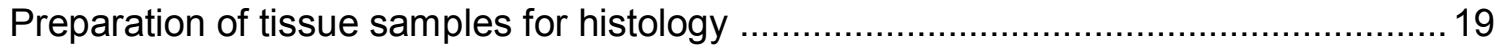

Luxol fast blue and periodic acid-Schiff (LFB-PAS) stain ........................................ 20

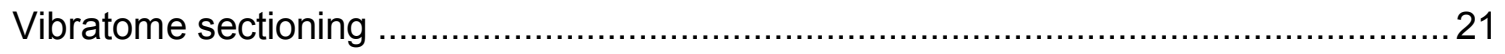

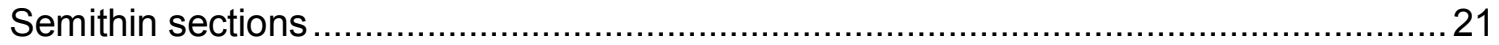

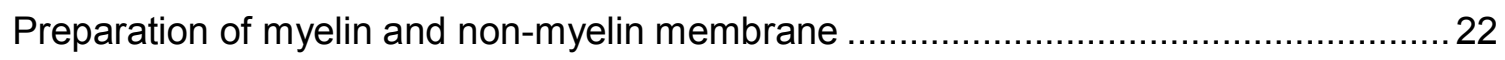

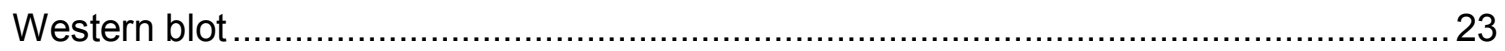

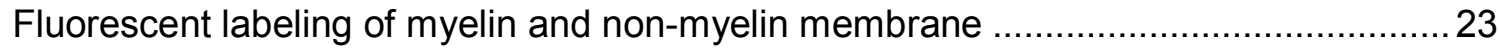

Fluorescence of PKH67-labeled myelin and non-myelin membrane ...........................24

Microglial cell culture, macrophage culture, and myelin treatment ..............................24

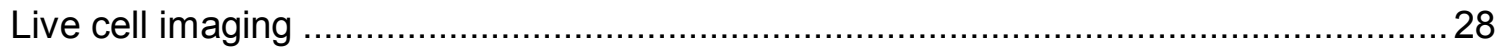

Organotypic hippocampal slice culture and ex vivo demyelination models....................29

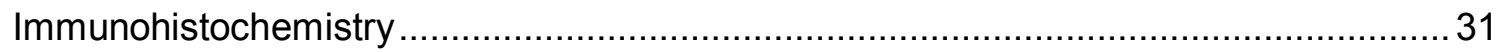

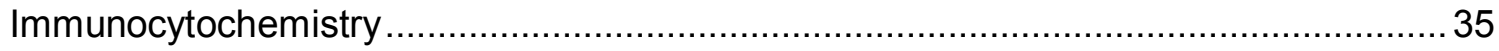

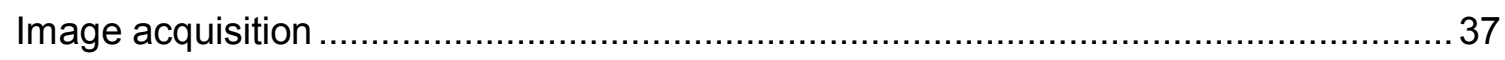

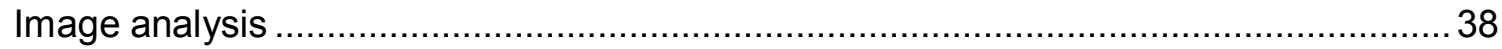

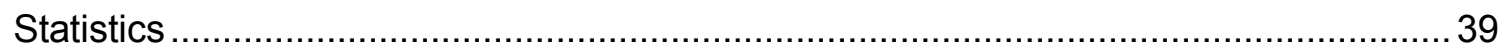

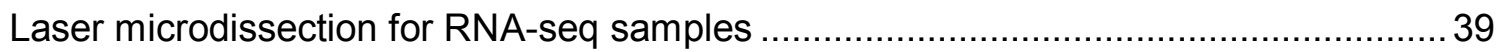

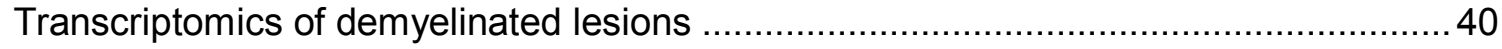

Proteomics and pathway analysis of cultured microglia ......................................... 40

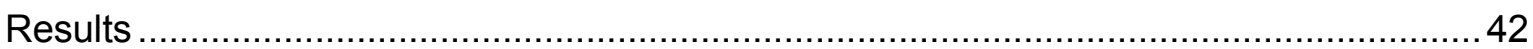

Remyelination was impaired in the spinal cord of $M y d 88^{-/-}$mice ................................ 42

Microglia / macrophages were recruited to demyelinated lesions .............................. 44 
Astrogliosis was not affected in demyelinated lesions of $M y d 88^{-/-}$mice

Myelin debris was taken up faster and in larger amounts than non-myelin membrane by

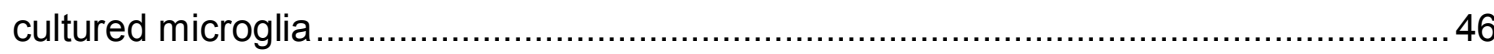

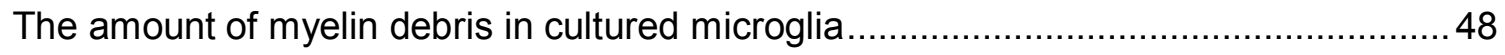

Maturation of phagosomes containing myelin debris in cultured microglia ................... 49

Proteomic analysis of cultured microglia after exposure to myelin debris .....................52

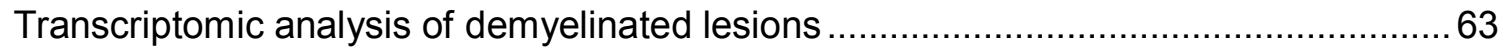

Assay for myelination in organotypic hippocampal slice culture (OHSC)....................64

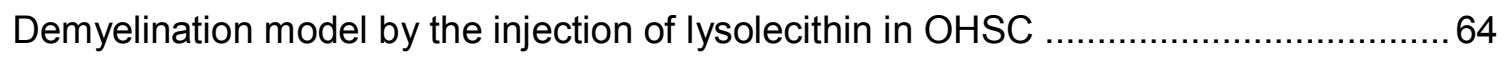

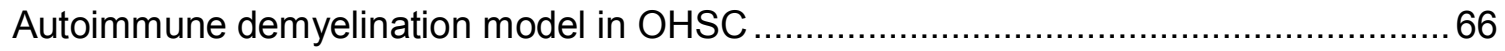

Oligodendrocyte development in Myd88-knockout mice .........................................6 68

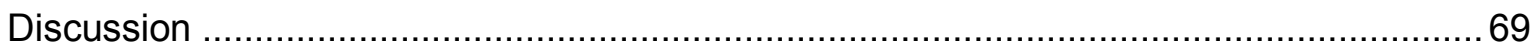

MyD88-dependent pathways are necessary for the recruitment of OPCs and

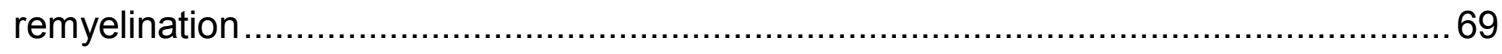

MyD88-deficient microglia have impaired phagosome maturation after the phagocytosis

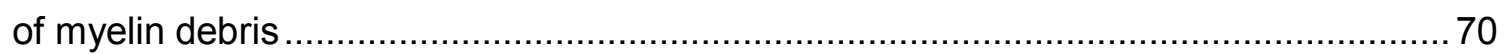

Microglia activation and secretion during remyelination ............................................. 73

Validation of candidate molecules for the effects on OPC response ..........................78

Lack of remyelination in organotypic hippocampal slice culture .................................79

MOG antibody and complement-mediated demyelination in OHSC .......................... 80

The MyD88-dependent recruitment of OPCs may be specific to remyelination in the

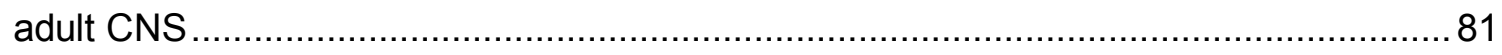

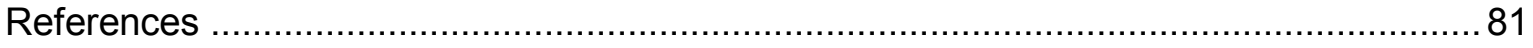

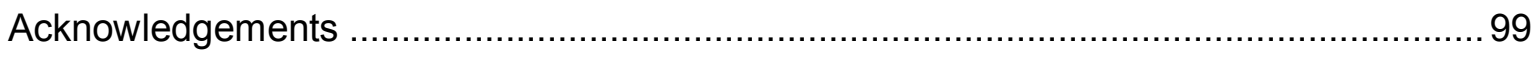

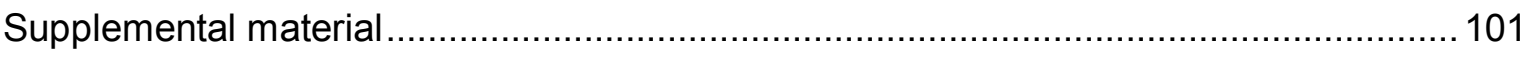

Activation of cultured microglia / macrophages after the exposure to myelin debris ...101

OPC proliferation measured by EdU in tissue culture ............................................. 101

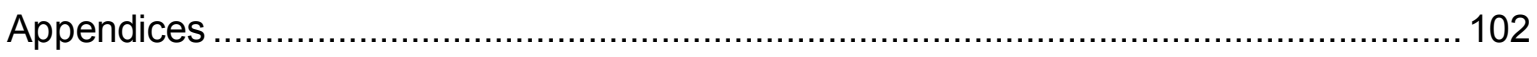

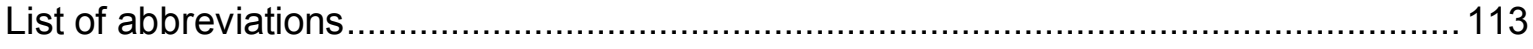

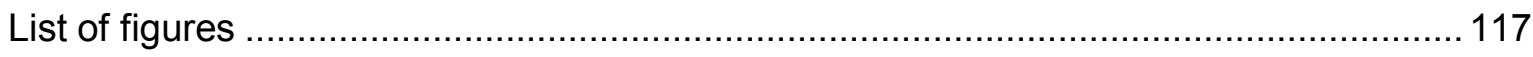


List of tables.

Declaration of authorship 120 


\section{Abstract}

In the central nervous system, remyelination requires the proliferation and migration of oligodendrocyte progenitor cells (OPCs) to the lesions, and the differentiation of OPCs into oligodendrocytes that remyelinate the axons. Microglia / macrophages remove damaged myelin from the lesions, mediate inflammation, and secrete cytokines and growth factors that regulate OPC responses for remyelination. In the mice deficient of MyD88 adaptor for inflammatory signaling, the recruitment of OPCs and remyelination were impaired after lysolecithin-induced focal demyelination. The lack of MyD88 signaling and defective phagosome maturation may impair the clearance of internalized myelin debris and the activation of microglia to support remyelination. Transcriptomic and proteomic analyses of demyelinated lesions and cultured microglia identified candidate molecules and pathways relevant to remyelination. Lysolecithin or autoimmune-mediated demyelination models in organotypic hippocampal slice culture provide ex vivo methods for understanding the mechanisms of demyelination and remyelination, and for evaluating the effects of candidate molecules on OPC proliferation, differentiation and myelination. In addition, MyD88 signaling is necessary for the recruitment of OPCs during remyelination in the adult CNS but may not be essential for developmental myelination.

\section{Introduction}

The central nervous system (CNS) is composed of a diverse set of cell types, including neurons, glia (astrocytes, oligodendrocytes and microglia) and vascular cells (endothelial cells, pericytes and vascular smooth muscle cells) (Zeisel et al., 2015; Zhang et al., 2014). Different cell types interact with each other during neural development and for normal functions of the nervous system. While neurons, astrocytes and oligodendrocytes originate from precursor cells derived from neuroectoderm (Allen and Barres, 2009), microglia are immune cells derived from progenitor cells that entered the nervous system during embryonic development (Ginhoux and Garel, 2018; Ginhoux et al., 2010). Microglia have versatile functions during development, including the regulation of neurogenesis, synaptic pruning, the refinement of synapses, and oligodendrocyte development. Microglia monitor the CNS to detect pathogens and clear damaged structures and dead cells, both of which use their phagocytosis machinery. These resident immune cells also mediate inflammation in the CNS. Under physiological conditions, adult microglia display ramified morphology with many processes, and each cell is located in its own territory; whereas activated microglia have enlarged cell body and shorter processes, and 
sometimes exhibit amoeboid morphology (Hammond et al., 2018; Hanisch and Kettenmann, 2007; Ransohoff and Perry, 2009). The general microglial markers such as IBA1 and CX3CR1 could not distinguish microglia from macrophages until recent studies identified specific markers that are only expressed by microglia such as SALL1 (Buttgereit et al., 2016; Gosselin et al., 2017) and TMEM119 (Bennett et al., 2016; Satoh et al., 2016). In the case of demyelination, both microglia and monocyte-derived macrophages are found in demyelinated lesions (Davies and Miron, 2018; Franklin and FfrenchConstant, 2017). Nonetheless, the functions of microglia in remyelination were the main focus of this study.

Myelin is the multilayered membrane wrapped around axons in the vertebrate nervous system. Myelin sheaths are formed by oligodendrocytes in the CNS, and by Schwann cells in the peripheral nervous system (PNS). Myelin insulates axons and restricts $\mathrm{Na}^{+}$ channels to the nodes of Ranvier, which results in the saltatory conduction of action potentials between the nodes. This allows fast nerve conduction in relatively thin axons and saves energy. Oligodendrocytes also provide trophic and metabolic support for axons through myelin, which helps to maintain axonal integrity and health (Bercury and Macklin, 2015; Kremer et al., 2016). Myelination in humans is most active in the first year of life and continues during young adulthood, especially in the prefrontal cortex (Sowell et al., 1999; Sowell et al., 2001). In mice, myelination starts at birth in the spinal cord, peaks between postnatal day (P) 10 and 20, and becomes almost completed by P60 in most brain regions. During development, oligodendrocyte progenitor cells (OPCs) proliferate and migrate out of the subventricular zone (SVZ), and differentiate into mature oligodendrocytes (Baumann and Pham-Dinh, 2001). The transcription factors OLIG2 and NKX-2.2 specify the cell fate of oligodendrocyte progenitors, and OLIG2 is expressed throughout the oligodendrocyte lineage (Xu et al., 2000; Zhou et al., 2001). OPCs express markers such as the chondroitin sulfate proteoglycan AN2/NG2 (Nishiyama et al., 1996) and the platelet-derived growth factor receptor alpha (PDGFR $\alpha$ ) (Hall et al., 1996). Early myelinating oligodendrocytes express the marker BCAS1 (Fard et al., 2017). Mature oligodendrocytes express markers such as APC (CC-1) (Lang et al., 2013), CNP, myelin basic protein (MBP) (Reynolds and Wilkin, 1988), proteolipid protein (PLP/DM20) (Nave et al., 1987), and myelin oligodendrocyte glycoprotein (MOG) (Pham-Dinh et al., 1993). Myelin is rich in lipids (around $70 \%$ of the dry weight) and cholesterol, whereas other types of membrane are made of roughly equal amounts of proteins and lipids (Chrast et al., 2011; Fledrich et al., 2018; O'Brien and Sampson, 1965). Myelination is a well- 
coordinated process to assemble membrane around axons, generating compacted myelin that supports the function of the nervous system (Snaidero and Simons, 2014).

In the most frequent demyelinating disease multiple sclerosis (MS), the damage to myelin causes the loss of saltatory nerve conduction and trophic and metabolic support for axons. Remyelination has been observed in humans in an early phase of the disease (Prineas et al., 1993; Prineas et al., 1989; Raine and Wu, 1993), but the regeneration is often not effective to prevent neurodegeneration and disability as the disease progresses (Franklin et al., 2012; Nave, 2010). MS is a putative autoimmune disease, supported by various lines of evidence for autoreactive T lymphocytes (Compston and Coles, 2008; International Multiple Sclerosis Genetics et al., 2011; Ludwin, 2000). Three general categories of experimental demyelination and remyelination models are studied to understand demyelinating diseases: (1) toxin-induced demyelination models cause damage to oligodendrocytes and primary demyelination without autoimmunity. Demyelination is separated from the subsequent remyelination process. The common reagents used to induce focal demyelination or kill oligodendrocytes are lysolecithin, cuprizone and ethidium bromide. (2) Autoimmune-induced models, for example, the experimental autoimmune encephalomyelitis $(E A E)$ involves the immunization of animals with CNS antigens such as myelin proteins or the injection of an autoantibody (Franklin and Ffrench-Constant, 2017; Kremer et al., 2016). For instance, MOG is a membrane protein expressed on oligodendrocytes and the outermost surface of myelin sheaths. The injection of anti-MOG antibody into rats can activate endogenous complement and mediate demyelination (Linington et al., 1988). (3) Viral models mimic some aspects of MS pathology (Kremer et al., 2016). In this study, the mechanisms of remyelination were investigated after toxin-induced focal demyelination by the injection of lysolecithin into the mouse spinal cord. In this model, demyelination is complete 4 days post-injection (DPI); the number of microglia / macrophages in the lesions peaks at $7 \mathrm{DPI}$; remyelination is most prominent between 14 and 21 DPI (Cantuti-Castelvetri et al., 2018; Jeffery and Blakemore, 1995; Miron et al., 2013)

Remyelination is a highly orchestrated process of generating new oligodendrocytes from OPCs and forming new myelin sheaths around demyelinated axons. In the lysolecithin model, the inflammatory response is the consequence of demyelination rather than the cause, and the depletion of macrophages using clodronate-liposomes resulted in impaired remyelination (Kotter et al., 2001). This is consistent with the notion that reparative inflammation prepares damaged tissue for regeneration (Karin and Clevers, 2016). Since 
microglia / macrophages are the professional phagocytes in the CNS, they are most likely to clear damaged myelin, detect demyelination, and secrete cytokines and signaling molecules to activate OPCs for remyelination (Miron, 2017). Myelin debris in the lesion can inhibit OPC differentiation and remyelination (Kotter et al., 2006; Lampron et al., 2015), and therefore it must be removed from the lesions. The generation of new oligodendrocytes is crucial, because genetic fate mapping showed that pre-existing mature oligodendrocytes do not contribute to remyelination (Crawford et al., 2016). In response to demyelination, adult OPCs change their gene expression to an immature state, and become activated for proliferation and migration to repopulate the lesion (Moyon et al., 2015). After the recruitment, OPCs differentiate into mature myelinating oligodendrocytes and regenerate myelin. This process is similar to developmental myelination in terms of the expression of oligodendrocyte markers at the corresponding stages, although regenerated myelin sheaths are thinner and shorter than intact myelin in the adult CNS (Capello et al., 1997; Ludwin and Sternberger, 1984; Morell et al., 1998; Sim et al., 2000). Remyelination is occasionally contributed by progenitors from the SVZ to nearby lesions, for instance, in the corpus callosum (Kazanis et al., 2017; Samanta et al., 2015; Xing et al., 2014). Astrocytes might also respond to the signals from microglia and secrete molecules that regulate OPCs and/or microglia (Hammond et al., 2014; Petkovic et al., 2016). The signals for OPC activation may also come from vascular cells (Arai and Lo, 2009), OPCs themselves (Moyon et al., 2015) and regulatory T cells (Dombrowski et al., 2017).

Neuropathological studies of MS lesions showed that around $30 \%$ of lesions contain few OPCs, whereas $70 \%$ of lesions have sufficient OPCs but they fail to differentiate into mature oligodendrocytes for remyelination (Boyd et al., 2013; Lucchinetti et al., 1999). Therefore, finding the molecules and/or pathways that recruit OPCs to the lesions as well as those that promote OPC differentiation will help the development of therapy for MS. Many factors have been identified to regulate OPCs directly or indirectly in these phases of remyelination (Gaesser and Fyffe-Maricich, 2016; Swiss et al., 2011). For example, OPCs form new glutamatergic synapses with demyelinated axons, and the axonal activity can stimulate AMPA and kainite receptors, causing OPCs to differentiate (Etxeberria et al., 2010; Gautier et al., 2015). CCN3 derived from regulatory T cells was shown to promote remyelination (Dombrowski et al., 2017). It was also demonstrated that CNTF and LIF enhance remyelination by activating the JAK2/STAT3 pathway (Steelman et al., 2016). LINGO-1 was identified to negatively regulate oligodendrocyte differentiation and myelination (Mi et al., 2005), and blocking LINGO-1 signaling improved remyelination (Mi 
et al., 2009). The activation of ERK1/2 MAP kinases in OPCs was shown to increase the thickness of myelin during development (Ishii et al., 2012) and to promote remyelination (Fyffe-Maricich et al., 2013; Jarjour et al., 2015; Michel et al., 2015; Najm et al., 2015). Retinoid X receptor RXR-y signaling was shown to promote OPC differentiation and remyelination by studying focal demyelination models in the rat CNS (Huang et al., 2011). CHD7 interacts with SOX10 and regulates myelination and remyelination (He et al., 2016). The transcription regulator Ascl1/Mash1 was indicated to increase oligodendrogenesis during development and remyelination (Nakatani et al., 2013). The important roles of microglia and macrophages in remyelination have been supported by the molecules derived from them that have effects on OPCs (Franklin and Ffrench-Constant, 2017). The pro-inflammatory cytokine interleukin (IL)-1 $\beta$ was shown to promote remyelination (Mason et al., 2001). In $I L 1 b^{-/}$mice, the lack of the secretion of IGF-1 by microglia / macrophages and astrocytes results in the delay of OPC differentiation and inadequate remyelination (Mason et al., 2003). Another pro-inflammatory cytokine TNF- $\alpha$ was indicated to promote OPC proliferation and remyelination after cuprizone-induced demyelination (Arnett et al., 2001) and to enhance oligodendrocyte differentiation and remyelination in EAE (Madsen et al., 2016). The upregulation of the chemokine CXCL12 was shown to activate its receptor CXCR4 on OPCs, promoting OPC differentiation and remyelination (Patel et al., 2010). Macrophage-derived endothelin 2 was identified to enhance remyelination (Yuen et al., 2013). The TGF- $\beta$ superfamily member activin-A secreted by microglia / macrophages was shown to induce oligodendrocyte differentiation during remyelination (Miron et al., 2013).

After microglia / macrophages internalize myelin debris, they need to clear the myelin debris and secrete the molecules to create a supportive environment for remyelination. The accumulation of myelin debris, especially cholesterol, in microglia / macrophages and impaired remyelination are characteristic of demyelinated lesions in old mice. Cholesterol transporters such as apolipoprotein E (APOE) are required for clearing myelin debris and resolving inflammation, and the stimulation of cholesterol efflux from the phagocytes can restore the remyelination capacity of old mice (Cantuti-Castelvetri et al., 2018). A study showed that systemic injections of amphotericin $B$ and macrophage colony-stimulating factor (M-CSF) activated microglia / macrophages, increased the number of OPCs and promoted remyelination. The stimulated production of TNF- $\alpha$ by macrophages was abolished in absence of both MyD88 and TRIF in vitro (Doring et al., 2015). This suggests that Toll-like receptor (TLR)/MyD88 signaling might be involved in the regulation of remyelination. 
The myeloid differentiation primary response protein MyD88 is the canonical adaptor protein for the inflammatory signaling pathways of TLRs and IL-1 receptor family (Deguine and Barton, 2014). TLRs are a family of pattern recognition receptors (PRRs) that sense pathogens or endogenous damage signals and initiate innate and adaptive immune responses. The TLRs located in the plasma membrane have extracellular domains that recognize pathogen- or damage-associated molecular patterns (PAMPs or DAMPs) from outside the cell, whereas the TLRs located in the endosome membrane recognize signals of intracellular PAMPs or DAMPs in the endosome. The activation of TLRs by its ligands leads to the interaction of TLRs with different combinations of adaptor proteins. While most TLRs (except TLR3) depend on MyD88 to initiate the signaling efficiently, the adaptor protein TRIF can also mediate the signaling of some TLRs (Kawai and Akira, 2006). The MyD88-mediated signaling of TLRs and IL-1 receptor family involves interactions among various signaling components and a series of phosphorylation and ubiquitination events, resulting in the activation of NF-KB and MAP kinase signaling, which induces the expression of inflammatory cytokines; and the activation of interferon regulatory factors (IRFs) for the expression of type I and III interferons (IFNs) (Ank et al., 2008; Kawai and Akira, 2006; Onoguchi et al., 2007). In the Myd88 ${ }^{-/}$mice, the induction of cytokines and IFN-y by IL-1 and IL-18 was abolished (Adachi et al., 1998), but the activation of NF-KB and MAP kinases in response to TLR2 and TLR4 signaling was still observed (Kawai et al., 1999). MyD88 is primarily expressed in microglia / macrophages in the CNS. The Myd88 mRNA expression levels in various cell types in the cerebral cortex are: microglia / macrophages $46.2712 \pm 3.289$ FPKM, endothelial cells $8.81965 \pm$ 0.42895 FPKM, OPCs $3.0818 \pm 0.034$ FPKM, astrocytes $3.0226 \pm 0.2468$ FPKM, myelinating oligodendrocytes $1.94655 \pm 0.19675 \mathrm{FPKM}$, newly formed oligodendrocytes $1.9381 \pm 0.1961 \mathrm{FPKM}$, and neurons $1.3718 \pm 0.1719$ FPKM (Brain RNA-Seq) (Zhang et al., 2014). Therefore, microglia / macrophages are the main cell types affected by MyD88 deficiency in the CNS.

In my Ph.D. project, remyelination was examined in $M y d 88^{-/-}$mice to investigate the roles of microglia activation and inflammatory response. Fewer OPCs and impaired remyelination were observed in $M y d 88^{-/}$mice after lysolecithin-induced demyelination. To understand the cause of impaired remyelination, the phagocytosis of damaged myelin, phagosome maturation and the clearance of internalized myelin debris by microglia were studied. Furthermore, the activation phenotypes of microglia during remyelination, and the relay of signals to regulate the responses of OPCs for remyelination were compared between wild-type (WT) and Myd88^- microglia. 
Phagocytosis is a process to internalize large particles that are $\geq 0.5 \mu \mathrm{m}$ (Allen and Aderem, 1996). In metazoans, phagocytosis is a critical defense mechanism of innate immunity, and contributes to the maintenance of homeostasis by clearing damaged structures and dead cells. The particles are recognized by phagocytic receptors on the cell surface, which initiates signaling pathways that lead to the remodeling of actin cytoskeleton and the formation of phagocytic cups (Pauwels et al., 2017). The phagosomes containing internalized particles are moved along microtubules, and actin polymerizes at the phagosomal membrane (Blocker et al., 1997). During phagosome maturation, phagosomes interact with early endosomes and late endosomes, forming early phagosomes and late phagosomes, respectively, and eventually fuse with lysosomes, forming phagolysosomes (Pauwels et al., 2017). Phagosomes acquire the vacuolar-type $\mathrm{H}^{+}$-ATPases (v-ATPases), which acidifies their lumen (Lukacs et al., 1990), and the NADPH oxidase complex, which produces reactive oxygen species (Savina et al., 2006). The low pH in the lumen of phagolysosomes allows optimal activity of hydrolytic enzymes such as cathepsins for the degradation of phagocytic cargos (Yates et al., 2005). Phagosome maturation is elegantly regulated at each step (Levin et al., 2016), and the lysosome-associated membrane glycoprotein 2 (LAMP-2) has been shown to be crucial for the maturation of phagosomes and autophagosomes (Beertsen et al., 2008; Huynh et al., 2007). Different kinetics of phagosome maturation serves different functions, depending on the internalized materials and the cell type. In macrophages, fast phagosome maturation enhances the killing of pathogens and the digestion of damaged materials (Yates et al., 2005); whereas in dendritic cells, delayed phagosome maturation allows antigen presentation of partially digested cargos to T lymphocytes (Savina and Amigorena, 2007). Late endosomes and lysosomes are often detected using LysoTracker ${ }^{\circledR}$ probes, which are weakly basic amines conjugated to a fluorophore. LysoTracker selectively accumulates in organelles with low luminal $\mathrm{pH}$ and generates enhanced fluorescence (Blander and Medzhitov, 2004; Kinchen and Ravichandran, 2008).

Phagosome maturation is a key step to deliver internalized pathogens or damaged materials from phagosomes to lysosomes for degradation. It has been shown that MyD88 is required for the phagocytosis of bacteria and phagosome maturation in macrophages. Bacteria, but not apoptotic cells, activated MyD88-dependent TLR signaling, which regulated phagosome maturation (Blander and Medzhitov, 2004). While the dependence of phagosome maturation on TLR2/4 signaling was controversial, Myd88 ${ }^{-/}$macrophages were confirmed to have impaired phagocytosis of pathogens and defective fusion of phagosomes and lysosomes (Henneke et al., 2002; Liu et al., 2004; Marr et al., 2003; 
Yates and Russell, 2005). The studies of phagocytosis were performed in serum-free medium to exclude the effects of Fc or complement receptors (Caron and Hall, 1998; Underhill and Ozinsky, 2002). In this study, the phagocytosis of myelin debris and phagosome maturation in WT and $M y d 88^{-/}$cultured microglia were analyzed, because they are critical for the degradation of internalized myelin debris and the activation phenotype(s) of microglia.

The microglia isolated from healthy brains show a core gene expression profile (Bennett et al., 2016; Butovsky et al., 2014; Hickman et al., 2013; Wes et al., 2016). Upon activation, individual microglia exhibit mixed phenotypes that do not necessarily fit into either M1 (pro-inflammatory) or M2 (anti-inflammatory or immunoregulatory) phenotypes (Hanisch and Kettenmann, 2007; Ransohoff, 2016; Town et al., 2005). Microarray analysis of microglia after cuprizone-induced demyelination and remyelination identified the expression of some M1 and M2 markers, upregulation of MHC class II, and the genes for microglial effector functions, including phagocytosis, lipid recycling, tissue remodeling, and the factors that promote the recruitment or differentiation of OPCs. However, the study did not identify novel secreted factors or pathways that are supportive for remyelination (Olah et al., 2012). In contrast to bulk transcriptomics that measures the ensemble gene expression of a population, possibly averaging out the differences among subsets of microglia, single-cell transcriptomics has identified distinct microglia activation phenotypes in recent studies (Keren-Shaul et al., 2017). For instance, single-cell RNA sequencing (RNA-seq) of microglia during the progression of neurodegeneration in mice identified disease stage-specific cell states, including two reactive microglia phenotypes the early response microglia that express the signature of type I IFN response genes, and the late response microglia that show the upregulation of MHC class II genes, which could be induced by IFN-Y (type II IFN) (Mathys et al., 2017). Pathway and network analysis of transcriptomic or proteomic data predicts models of regulatory networks, which could be potential hypotheses for mechanistic studies. For example, Ingenuity Pathway Analysis of the transcriptomes of the microglia isolated from mice during aging and in neurological disease models identified TGF- $\beta$ and APOE as the major upstream regulators of the homeostatic phenotype and the neurodegenerative phenotype, respectively, which was validated by experimental evidence (Krasemann et al., 2017).

The activation of microglia / macrophages in demyelinated lesions was analyzed by immunohistochemistry for the activation markers that are known to be relevant to remyelination. The ionized calcium binding adaptor molecule 1 (IBA1, encoded by the Aif1 
gene) is a general marker for microglia and macrophages. IBA1 is involved in the Rac signaling pathway that regulates actin cytoskeleton dynamics, and the expression level of IBA1 correlates with cell activation (Imai et al., 1996; Imai and Kohsaka, 2002). Galectin-3 (formally called MAC-2; encoded by the Lgals3 gene) is a carbohydrate-binding protein expressed by microglia / macrophages during remyelination. It was suggested to activate the phagocytosis of damaged myelin after injury (Rotshenker, 2009). Galectin-3 was also shown to be required for OPC differentiation and remyelination (Hoyos et al., 2014; Pasquini et al., 2011). The expression of major histocompatibility complex (MHC) class II by microglia / macrophages has been observed under physiological and pathological conditions. The MHC class II-positive microglia may not be involved in antigen presentation, for which co-stimulatory molecules are also required (Perry, 1998). The TNF- $\alpha$-dependent induction of MHC genes was identified in microglia and astrocytes during remyelination, and $\mathrm{MHC}$ class II-null mice displayed a delay in oligodendrocyte regeneration and remyelination (Arnett et al., 2003). The expression of other activation markers could also be analyzed. For example, the inducible nitric oxide synthase (iNOS) catalyzes the synthesis of nitric oxide from arginine during inflammation and is considered an "M1" marker (Sparrow, 1994). On the other hand, arginase-1 catalyzes the conversion of arginine to ornithine in competition with iNOS, and it is considered an "M2" marker. The expression of arginase- 1 in microglia is associated with the phagocytosis of apoptotic cells and damaged structures (Ahn et al., 2012). LAMP-2 (Mac-3 or CD107b), the lysosomal membrane glycoprotein required for the maturation of phagosomes and autophagosomes, is another activation marker of microglia / macrophages (Hagemeyer et al., 2017).

To identify the molecules and pathways involved in remyelination, transcriptomic and proteomic studies were performed to compare the gene expression profiles of the lesions or cultured microglia of WT and $M y d 88^{-/-}$mice. Cell culture assays were established for the analysis of secreted molecules from microglia after the exposure to myelin debris, or for the analysis of microglia activation phenotype after extended exposure to myelin debris. The experiments were carried out in defined, serum-free medium to minimize artificial activation of microglia by serum (Bohlen et al., 2017). Pathway analysis of the proteomic data of microglial cell lysate was conducted using Ingenuity Pathway Analysis (IPA) and the gene ontology enrichment analysis and visualization tool (GOrilla). IPA predicts regulatory networks and causal relationships associated with the gene expression data, based on individual relationships curated from the literature. "Upstream regulators" are molecules that might potentially explain the observed changes in gene expression in the dataset. They might be interdependent and form "mechanistic networks". The 
statistical significance of the regulators is scored by $p$-value and $z$-score. Here, $p$-value is an enrichment score that indicates the overlap of the observed and predicted regulated gene sets. The activation $z$-score infers the activation state of a putative regulator: $z<0$ suggests an inhibited regulator, and $z>0$ suggests an activated regulator (Kramer et al., 2014). GOrilla identifies enriched gene ontology terms and visualizes the analysis as a hierarchical structure. It computes a $p$-value for the observed enrichment based on a theoretical characterization of the distribution (Eden et al., 2009). Candidate molecules identified in the transcriptomic and proteomic analyses can be tested for their effects on OPC proliferation, differentiation and myelination in cell culture or organotypic slice culture assays.

Organotypic slice cultures are ex vivo models that maintain the cell populations and cellular architecture. Slice cultures are commonly used for studying neurodevelopment, brain damage and repair, and neuronal-glial interactions and so on. They are accessible for treatment, electrophysiology, and live imaging, etc. Slice cultures are prepared from early postnatal mice and are maintained using the interface method for up to one month (Stoppini et al., 1991). The method to deplete and replenish microglia in organotypic hippocampal slice culture (OHSC) provides a model for studying microglia of various genotypes in a culture system where they display in vivo-like ramified morphology (Masuch et al., 2016). Remyelination assay has been established in organotypic cerebellar slice culture (OCSC) and widely used for screening small molecules (Najm et al., 2015; Zhang et al., 2011). The assays for OPC proliferation and myelination were established in OHSC after comparing the responses of OHSC and OCSC. Proliferating cells are labeled with 5-ethynyl-2'-deoxyuridine (EdU), a thymidine analog that is incorporated into newly synthesized DNA (Salic and Mitchison, 2008). EdU, which contains an alkyne functional group, is detected by covalent linkage to a fluorophore with an azide group using the click reaction (Breinbauer and Kohn, 2003; Rostovtsev et al., 2002; Wang et al., 2003). The myelination assay was established using 3,3',5-triiodo-Lthyronine (T3), a thyroid hormone that promotes oligodendrocyte differentiation and myelination (Almazan et al., 1985; Baas et al., 1997; Jones et al., 2003). In addition, toxinand autoimmune-induced demyelination models were established in OHSC, which may be applied to study the mechanisms of demyelination and to test candidate molecules.

Since remyelination often recapitulates some aspects of developmental myelination, we asked whether MyD88-dependent pathways are required for the proliferation and recruitment of OPCs for myelination during postnatal development. A subset of amoeboid 
microglia that highly express LAMP-2 appears postnatally in the developing white matter of corpus callosum and cerebellum. Characterization of this microglia subset indicated the important roles of postnatal microglia in the development and homeostasis of OPCs (Hagemeyer et al., 2017). Therefore, the number of OPCs in the corpus callosum was analyzed at the age of the peak of amoeboid LAMP $-2^{+}$microglia to examine oligodendrocyte development in MyD88-deficient mice.

\section{Materials and methods}

\section{Mice}

All animal experiments were performed according to the German animal welfare law and local regulations for animal experimentation. The $M y d 88^{-/}$mice (Adachi et al., 1998) were generously provided by the Laboratory of Prof. Dr. Uwe-Karsten Hanisch in Göttingen. The Myd88 ${ }^{-/}$strain was kindly provided by the Laboratory of Dr. Arthur Liesz and bred as homozygotes in the animal facility of the Center for Stroke and Dementia, Munich.

\section{Genotyping}

The genotype of $M y d 88^{-/}$mice was confirmed by genotyping. Genomic DNA was extracted from the tissue of ear punches or a small fraction of the animals' tail using the Invisorb ${ }^{\circledR}$ Spin Tissue Mini Kit (Stratec Molecular) according to the user manual and the following modifications: the tissue was lysed with $400 \mu \mathrm{L}$ of Lysis Buffer $\mathrm{G}$ and $40 \mu \mathrm{L}$ of Proteinase $\mathrm{S}$ or $\mathrm{K}$ (vortexed for $5 \mathrm{sec}$ ) at $52^{\circ} \mathrm{C}$ overnight. The centrifugation to remove residual ethanol was done at $11000 \times g$ for 6 min. DNA was collected by incubation with $100 \mu \mathrm{L}$ of Elution Buffer for $3 \mathrm{~min}$ and centrifugation at $11000 \times \mathrm{g}$ for $1 \mathrm{~min}$.

The DNA fragments were amplified by polymerase chain reaction (PCR) using GoTaq ${ }^{\circledR}$ G2 DNA polymerase (Promega, M7845). The genotyping protocol for the Myd88 ${ }^{-/}$strain was modified from the protocol provided by the Laboratory of Dr. Arthur Liesz. 


\begin{tabular}{|c|l|c|c|}
\hline Oligo Name & Sequence 5' to 3' & Scale $(\mu \mathrm{mole})$ & Purification \\
\hline Myd88F & GTT GTG TGT GTC CGA CCG T & 0.025 & Desalted \\
\hline Myd88R & GTC AGA AAC AAC CAC CAC CAT GC & 0.025 & Desalted \\
\hline
\end{tabular}

The reaction mix was prepared as the following:

\begin{tabular}{lr} 
Reaction mix for 1 reaction & $20 \mu \mathrm{L}$ \\
\hline Autoclaved deionized $\mathrm{H}_{2} \mathrm{O}$ & $13.5 \mu \mathrm{L}$ \\
$5 \times$ buffer & $4 \mu \mathrm{L}$ \\
$2 \mathrm{mM}$ dNTP & $1 \mu \mathrm{L}$ \\
$10 \mu \mathrm{M}$ Myd88F & $0.2 \mu \mathrm{L}$ \\
$10 \mu \mathrm{M}$ Myd88R & $0.2 \mu \mathrm{L}$ \\
GOTaq DNA polymerase & $0.1 \mu \mathrm{L}$ \\
DNA & $1 \mu \mathrm{L}$
\end{tabular}

The reaction was done in a T3000 Thermocycler (Biometra) using the following program:

$94{ }^{\circ} \mathrm{C} \quad 3 \mathrm{~min}$

\begin{tabular}{|c|c|}
\hline $94^{\circ} \mathrm{C}$ & $30 \mathrm{~s}$ \\
\hline $66^{\circ} \mathrm{C}$ & $1 \mathrm{~min}$ \\
\hline $72{ }^{\circ} \mathrm{C}$ & $1 \mathrm{~min}$ \\
\hline
\end{tabular}

$4{ }^{\circ} \mathrm{C} \quad \infty$

The genotype was identified by agarose gel electrophoresis of the PCR product. The expected size of DNA fragment amplified from Myd88-knock out is 353 base pairs (bp), and 266 bp from WT. On a $2 \%$ agarose gel (prepared in $1 \times$ TAE buffer) containing GelRed $\circledast$ Nucleic Acid Gel Stain ( $2 \mu \mathrm{L}$ per $150 \mu \mathrm{L}$ of gel) (Biotium, 41003), $4 \mu \mathrm{L}$ of PCR product, $4 \mu \mathrm{L}$ of GeneRuler 100 bp DNA Ladder (Thermo Scientific ${ }^{\mathrm{TM}}$, SM0244) and $1 \mu \mathrm{L}$ of Tracklt ${ }^{\mathrm{TM}} 25$ bp DNA Ladder (Invitrogen ${ }^{\mathrm{TM}}$, 10488022) were loaded. The electrophoresis was run at 100-120 V for $\sim 60 \mathrm{~min}$ (until the yellow band almost reached the end of the gel). The gel was imaged by the exposure to UV light using the gel documentation system, and the images were printed and exported as TIFF using the Image Lab ${ }^{\text {TM }}$ software. 


\section{Lysolecithin-induced demyelination}

Stereotactic injection of lysolecithin in the spinal cord was performed in $M y d 88^{-/-}$mice that were 9-12 weeks old and age-matched wild-type C57BL/6J mice after at least 2 weeks of acclimatization to the animal unit.

Glass Capillaries for Nanoliter 2010, fire polished 2 (World Precision Instruments, 504949 or 4878) were pulled using the P-1000 Next Generation Micropipette Puller (Sutter Instrument) (in Göttingen). The program had the following parameters: Heat 530, Pull 0, Vel 60, Time 250, Pressure 500, Ramp 520, Microinjection - BF100.50.10, Tip < $1 \mu \mathrm{m}$, Taper 6-8 mm. R 40-80 Meg, Heat = Ramp, FB255B, 2.5mm Box. Alternatively, the capillaries were pulled using the DMZ-Universal Puller (Laboratory of Dr. Bettina Schmid, Munich). The $P(A) 60$ program is the following:

\begin{tabular}{|l|l|l|l|}
\hline HEAT & H 401 & T(F1) 250 & DELAY Pull1 \\
\hline FORCE Pre-pull & F (TH) 025 & F1 400 & FORCE Pull1 \\
\hline DISTANCE Threshold & D s(TH) 028 & s (F2) 010 & DISTANCE Pull2 \\
\hline DELAY Heatstop DIST. R-Polish & $\mathrm{T}(\mathrm{H}) 006$ & F2 200 & FORCE Pull2 \\
\hline DISTANCE Heatstop DIST. L-Polish & $\mathrm{s}(\mathrm{H}) 000$ & AD 000 & ADJUST \\
\hline
\end{tabular}

The reagents for injection were prepared under the cell culture hood. 1\% lysolecithin was prepared by dissolving L-a-Lysophosphatidylcholine from egg yolk (Sigma, L4129) in PBS, pH 7.4 (Gibco ${ }^{\mathrm{TM}}$, 10010056). 3\% Monastral blue was prepared by dissolving Copper(II) phthalocyanine-tetrasulfonic acid tetrasodium salt (Aldrich, 274011) in deionized water, and the solution was passed through $0.45-\mu \mathrm{m}$ filter and autoclaved. Prior to injection, $1 \mu \mathrm{L}$ of $3 \%$ Monastral blue was mixed with $25 \mu \mathrm{L}$ of $1 \%$ lysolecithin.

Before surgery, the animals were anesthetized by intraperitoneal (IP) injection with $150 \mu \mathrm{L}$ of ketamine/xylazine (10 mg/mL ketamine and $1 \mathrm{mg} / \mathrm{mL}$ xylazine) or $400 \mu \mathrm{L}$ of avertin (in Göttingen). Avertin was prepared by dissolving $1 \mathrm{~g}$ 2,2,2-tribromoethanol (Aldrich, T48402) in $0.81 \mathrm{~mL}$ of tert-amyl alcohol and $71.49 \mathrm{~mL}$ of deionized water by stirring and heating at $\sim 40{ }^{\circ} \mathrm{C}$. The solution was passed through a $0.22-\mu \mathrm{m}$ filter, aliquoted, and stored protected from light at $4{ }^{\circ} \mathrm{C}$ for maximum 1 week or at $-20^{\circ} \mathrm{C}$. Alternatively (in Munich), the animals were anesthetized by IP injection of $0.5 \mathrm{mg} / \mathrm{kg}$ body weight medetomidine, 5.0 $\mathrm{mg} / \mathrm{kg}$ midazolam and $0.05 \mathrm{mg} / \mathrm{kg}$ fentanyl (MMF). The anesthetized animals were kept 
on a heating pad at $37^{\circ} \mathrm{C}$, and Bepanthen eye ointment was applied to prevent drying of eyes. The anesthetic depth was monitored by checking the reflex between the toes and the corneal reflex.

The surgery and intraspinal injection of lysolecithin was conducted using the digital mouse stereotaxic frame and Nanoliter 2010 Injector with MICRO4 controller (World Precision Instruments) as previously described (Cantuti-Castelvetri et al., 2018). After the spinal cord was exposed, the capillary was positioned $0.55 \mathrm{~mm}$ lateral to the dorsal artery, and lowered $1.15 \mathrm{~mm}$ into the tissue. At each injection site, $1 \mu \mathrm{L}$ of $1 \%$ lysolecithin containing $0.12 \%$ Monastral blue was injected at a speed of $150 \mathrm{~nL} / \mathrm{min} .1 \mathrm{~min}$ after lysolecithin was delivered, the capillary was slowly retracted. After injection, the skin was sutured, and the wound was disinfected and sealed with the tissue adhesive Histoacryl $(B$. Braun). After the operation, the animals were injected IP with $250 \mu \mathrm{L}$ of $0.9 \% \mathrm{NaCl}$ (normal saline solution) to compensate for the loss of blood. The analgesic buprenorphine was injected subcutaneously (SC) at a dose of $0.1 \mathrm{mg} / \mathrm{kg}$. When MMF was used for anesthesia, 2.5 $\mathrm{mg} / \mathrm{kg}$ atipamezole, $0.5 \mathrm{mg} / \mathrm{kg}$ flumazenil and $1.2 \mathrm{mg} / \mathrm{kg}$ naloxone (AFN) was injected IP for the animals to wake up. The animals were kept on a heating pad at $37^{\circ} \mathrm{C}$ until they were awake. They were supplied with wet powder food in a 100-mm Petri dish in addition to regular food and water. The wet powder food was refreshed daily until the animals' hind limbs could function properly. The animals were injected SC with buprenorphine one or two days after surgery. Dr. Ludovico Cantuti-Castelvetri helped me with many stereotactic injection experiments.

\section{Preparation of tissue samples for histology}

The mice were fixed by transcardial perfusion to prepare samples for histology. Adult animals were anesthetized by IP injection with $100-200 \mu \mathrm{L}$ of $14 \%$ chloral hydrate (prepared in water) (Sigma, C8383). Postnatal day (P) 7 mouse pups were anaesthetized by IP injection with $10 \mu \mathrm{L}$ of MMF. The blood was washed out by transcardial perfusion with filtered, ice-cold phosphate buffered saline (PBS, Table 4) for 5 min until clear liquid came out of the right atrium and the liver turned pale. The animals were then perfused with fresh, filtered, ice-cold 4\% paraformaldehyde (PFA) in PBS for 10-15 min (adult mice) or 3-5 min (P7 pups) until the animals stiffened. The brain was taken out of the skull and post-fixed in $4 \%$ PFA at $4{ }^{\circ} \mathrm{C}$ overnight (adult brains) or for maximum 6-8 $\mathrm{h}$ (pup brains). The spine was taken out. The bones on the ventral side were removed to expose the 
ventral spinal cord, and then the spine was fixed in $4 \%$ PFA at $4{ }^{\circ} \mathrm{C}$ for $3-6$ days. If only the spinal cord was needed for histology, the mice were anesthetized by IP injection with chloral hydrate or in a $\mathrm{CO}_{2}$ chamber, and euthanized by decapitation. The spine was dissected and fixed in the same way.

After post-fixation, the tissue was washed in PBS and processed for cryoprotection. The tissue not required for processing could be stored in PBS containing $0.1 \%$ sodium azide at $4{ }^{\circ} \mathrm{C}$. The spinal cord was dissected out of the bone. The brain and spinal cord were immersed in $30 \%$ sucrose (prepared in PBS on the day of use) at $4{ }^{\circ} \mathrm{C}$ for 3 days, until the tissue sank to the bottom of the Conical tube. The tissue was immersed in 1:1 mixture of $30 \%$ sucrose in PBS and Tissue-Tek® O.C.T. Compound (Sakura) at room temperature (RT) with shaking at $400 \mathrm{rpm}$ overnight. The tissue was transferred to O.C.T. and incubated at RT for 4-5 h (spinal cord) or 1 day (brain). Afterwards the tissue was embedded in O.C.T. on dry ice. The frozen tissue was stored at -20 or $-80^{\circ} \mathrm{C}$.

Coronal sections of spinal cords and brains were cut at a thickness of $12 \mu \mathrm{m}$ using a cryostat. The sections were mounted on SuperFrost ${ }^{\mathrm{TM}}$ Plus Microscope Slides (Thermo Fisher Scientific). The adjacent spinal cord sections were mounted on alternating slides so that in each pair of slides, one slide was used for immunohistochemistry whereas the other was used for luxol fast blue stain. The slides were stored at -20 or $-80{ }^{\circ} \mathrm{C}$.

\section{Luxol fast blue and periodic acid-Schiff (LFB-PAS) stain}

$0.1 \%$ luxol fast blue (LFB) was prepared by dissolving $0.4 \mathrm{~g}$ solvent blue 38 (Carl Roth, 7709.2 ) in $400 \mathrm{~mL}$ of $95 \%$ ethanol and $2 \mathrm{~mL}$ of acetic acid (glacial). The solution was filtered. The slides of mounted sections were dried in a $37^{\circ} \mathrm{C}$ incubator for $30 \mathrm{~min}$, and washed with PBS for 5 min on a shaker. The sections were immersed in deionized water for $5 \mathrm{~min}$ (to remove the salts), and dehydrated in $50 \%, 70 \%, 90 \%$ and $100 \%$ ethanol for 5 min each. The sections were incubated with $0.1 \%$ LFB in a glass container tightly wrapped with aluminum foil (to avoid the evaporation of ethanol) overnight at $57^{\circ} \mathrm{C}$. The slides were cooled to RT. For the differentiation of white and gray matters, $50 \mathrm{~mL}$ of each of the following solutions were prepared freshly in deionized water: $95 \%$ ethanol, $0.05 \%$ lithium carbonate (Sigma, 13010) (heated slightly to dissolve), and 70\% ethanol. In a $50-$ $\mathrm{mL}$ Conical tube, dipped a slide in $95 \%$ ethanol and rinsed with distilled water. The slide was then dipped 5 times in $0.05 \% \mathrm{Li}_{2} \mathrm{CO}_{3}$, dipped 5 times in $70 \%$ ethanol, and rinsed with 
distilled water (Kluver and Barrera, 1953). The slide was examined under the microscope. If white and gray matters were not distinguishable, the cycle was repeated by dipping once or twice in each solution. After this step, the slides were kept in PBS.

After LFB stain, PAS stain was used to detect polysaccharides on inflammatory cells. $0.5 \%$ periodic acid (P0430) was prepared freshly in deionized water. The slides were washed with distilled water for 2 min on a shaker, incubated with fresh $0.5 \%$ periodic acid for $10 \mathrm{~min}$, and washed 3 times for 2 min each with deionized water. The slides were then incubated with Schiff's reagent (Merck Millipore, 1.09033.0500) (protected from light) for $15 \mathrm{~min}$, and washed 3 times for 2 min with deionized water (protected from light).

Afterwards the slides were left in a container under running distilled water from the tap for 15 min.

After LFB-PAS stain, the slides were dehydrated in $90 \%$ ethanol for $1 \mathrm{~min}$, in $100 \%$ ethanol for $1 \mathrm{~min}$, and in xylene for at least $5 \mathrm{~min}$ under the fume hood for histology. The slides were mounted with DePeX slide mounting medium and No. 1.5 coverslips.

\section{Vibratome sectioning}

The spinal cord was sectioned using a vibratome (Leica Biosystems): longitudinal sections were cut for the dissection of lesions for RNA sequencing; coronal sections were cut to prepare samples for semithin sections. $20 \%$ gelatin was prepared by dissolving gelatin powder (Merck Millipore, 1040781000) in PBS by stirring and heating at $60{ }^{\circ} \mathrm{C}$. Before vibratome sectioning, the aliquots of $20 \%$ gelatin were thawed from $-20{ }^{\circ} \mathrm{C}$ and heated at $37^{\circ} \mathrm{C}$ with shaking. A segment of spinal cord (shorter than $3 \mathrm{~mm}$ ) was freshly dissected from the mouse, and embedded in $20 \%$ gelatin in an embedding mold (Sigma, E41401EA) on ice. $200-\mu \mathrm{m}$ coronal sections were cut at a speed of $0.4-0.5 \mathrm{~mm} / \mathrm{s}$. The coronal sections were fixed with $\mathrm{K} \& \mathrm{~S}$ (4\% PFA and $2.5 \%$ glutaraldehyde in $\mathrm{K} \& \mathrm{~S}$ buffer) under a coverslip in a 24 -well plate at $4{ }^{\circ} \mathrm{C}$ until further processing.

\section{Semithin sections}

The semithin sections were prepared and stained, and the images were acquired by Dr. Ludovico Cantuti-Castelvetri. The spinal cord sections were embedded in Epon and cut at 
a thickness of $500 \mathrm{~nm}$ using the Leica Ultracut S ultramicrotome (Leica). Semithin sections were stained with methylene blue-azure II to visualize the lipid-rich area such as myelin.

\section{Preparation of myelin and non-myelin membrane}

Myelin and non-myelin membrane were isolated from the brains of 12 adult (at least 8 weeks old) wild-type C57BL/6 mice using the Beckman Optima XL-80 Ultracentrifuge with the SW 28 swinging-bucket rotor. The Ultra-Clear ${ }^{\mathrm{TM}}$ Thinwall $38.5 \mathrm{~mL}$ Tubes (Beckman Coulter, 344058) were sterilized with UV under the laminar hood. The HEPES-EDTA buffer was prepared as $10 \mathrm{mM}$ HEPES and $5 \mathrm{mM}$ EDTA, $\mathrm{pH} 7.4$, filtered and stored at 4 ${ }^{\circ} \mathrm{C}$. $0.32 \mathrm{M}$ and $0.85 \mathrm{M}$ sucrose solutions were prepared freshly in cold $10 \mathrm{mM}$ HEPES, pH 7.4 (HEPES buffer) and filtered. Deionized water was also filtered and kept cold.

The mice were euthanized in a $\mathrm{CO}_{2}$ chamber followed by decapitation. 3 or 4 brains were put in a Dounce homogenizer containing $4 \mathrm{~mL}$ of HEPES buffer. The brains were first homogenized mechanically using the Dounce tissue grinder set. The homogenate was transferred to a conical tube and topped up to $5 \mathrm{~mL}$ with HEPES buffer. The homogenate was then sonicated for 5 min using the Sonifier W-250 D (Branson). The brain homogenate was stored at $-20^{\circ} \mathrm{C}$.

The isolation and purification of myelin and non-myelin fraction were done at $4{ }^{\circ} \mathrm{C}$ or on ice. $5 \mathrm{~mL}$ of brain homogenate was overlaid on a stepwise 0.32 / $0.85 \mathrm{M}$ sucrose gradient in an ultracentrifugation tube. The first centrifugation was done at $23800 \mathrm{rpm}(54000 \times \mathrm{g})$ for $35 \mathrm{~min}$. The myelin fraction was collected from the interphase using a P1000 pipet, whereas the non-myelin fraction in the pellet was resuspended with PBS. Deionized water was added to the myelin and non-myelin fractions to remove small membrane fragments by osmotic shock. Myelin and non-myelin fraction were pelleted by centrifugation at 23800 rpm $(54000 \times g)$ for $18 \mathrm{~min}$. The supernatant was aspirated carefully, and the pellets were resuspended with water. Myelin and non-myelin fraction were washed with water and centrifuged at $9500 \mathrm{rpm}(9000 \times \mathrm{g})$ twice for $18-22 \mathrm{~min}$. The supernatant was removed; 1 $\mathrm{mL}$ of HEPES buffer was added to each pellet, and the pellets were kept at $-20{ }^{\circ} \mathrm{C}$ until the purification steps. The crude products of myelin and non-myelin fraction were purified by repeating the stepwise density gradient centrifugation, osmotic shock and two washing steps. The HEPES buffer for cell culture was prepared from the $1 \mathrm{M} \mathrm{HEPES} \mathrm{(Gibco}{ }^{\mathrm{TM}}$, 
15630056), and the $\mathrm{pH}$ was measured by $\mathrm{pH}$-indicator strips (Merck Millipore, 109543) and adjusted to 7.4 with sodium bicarbonate $7.5 \%$ solution (Thermo Fisher Scientific, 25080). Each final pellet was resuspended with $500 \mu \mathrm{L}$ of HEPES buffer for cell culture. The myelin and non-myelin fraction were passed through a syringe attached to a $27 \mathrm{G}$ cannula. The concentration of proteins in myelin and non-myelin fraction were measured by Bradford assay (Bradford, 1976).

\section{Western blot}

The purity of non-myelin fraction was checked by western blotting for myelin basic protein (MBP). The proteins in myelin and non-myelin fraction were separated by polyacrylamide gel electrophoresis (PAGE) at a constant voltage of $100 \mathrm{~V}$ in stacking gel and $120 \mathrm{~V}$ in $13 \%$ resolving gel, and transferred to nitrocellulose membrane at a constant current of $350 \mathrm{~mA}$ for $1.5 \mathrm{~h}$. After blocking with 5\% milk in PBST (PBS containing $0.05 \%$ Tween-20) for $1 \mathrm{~h}$ at $\mathrm{RT}$, the membrane was incubated with polyclonal rabbit anti-human MBP (1:2500, Dako, A0623) primary antibody in $5 \%$ milk in PBST overnight at $4{ }^{\circ} \mathrm{C}$. The membrane was washed three times with PBST for $10 \mathrm{~min}$, and then incubated with HRPconjugated goat anti-rabbit IgG secondary antibody (1:5000, Jackson ImmunoResearch) in $5 \%$ milk in PBST for $1 \mathrm{~h}$ at RT, followed by at least three times of washing with PBST for $5 \mathrm{~min}$. The membrane was incubated with ECL substrate for $1 \mathrm{~min}$ on a shaker, exposed to autoradiograph film for $5 \mathrm{~min}$ and developed.

\section{Fluorescent labeling of myelin and non-myelin membrane}

Myelin and non-myelin membrane were labeled using the PKH67 Green Fluorescent Cell Linker Mini Kit (Sigma, MINI67). For each 200-300 $\mu$ g myelin or non-myelin fraction in 250 $\mu \mathrm{L}$ HEPES buffer for cell culture, a mixture of $750 \mu \mathrm{L}$ of Diluent $\mathrm{C}$ and $4 \mu \mathrm{L}$ of PKH67 (vortexed for mixing) was added. The mixture was incubated for 5 min at RT (protected from light) and centrifuged at $15000 \times g$ for $10 \mathrm{~min}$ at $4{ }^{\circ} \mathrm{C}$. The pellet was resuspended with $250 \mu \mathrm{L}$ of HEPES buffer or PBS for cell culture, and passed through a $27 \mathrm{G}$ cannula. 


\section{Fluorescence of PKH67-labeled myelin and non-myelin membrane}

The fluorescence of PKH67-labeled myelin and non-myelin fraction was measured at an excitation wavelength of $485 \mathrm{~nm}$ and emission wavelength of $535 \mathrm{~nm}$ using the Perkin Elmer Wallac 1420 Victor $^{\mathrm{TM}}$ microplate reader. The samples of labeled myelin and nonmyelin fraction were diluted to a protein concentration of 1 and $0.1 \mu \mathrm{g} / \mathrm{mL}$ by serial dilution, and the fluorescence emission was measured in quadruplicates.

\section{Microglial cell culture, macrophage culture, and myelin treatment}

Primary microglial cell culture was prepared by the shake of mixed glial culture in the first half of my doctoral study. We switched to the culture of microglia isolated by magneticactivated cell sorting (MACS), where the morphology of the cells looked more similar to microglia in vivo. In both culture methods, microglia were cultivated in the DMEM/FCS/L929 medium (components see Table 1) until the cells were ready for experiment. The microglia (shake) cultures were treated in DMEM/FCS medium. The MACS microglia cultures were preferably washed and incubated with the serum-free TCC (or TIC) medium for $3 \mathrm{~h}$ to overnight before experiment, and treated in TCC medium (Bohlen et al., 2017). To improve the attachment of cells, No. 1.5 coverslips were treated with hydrochloric acid (fuming) overnight, washed at least 10 times with distilled water, dried and disinfected at $200{ }^{\circ} \mathrm{C}$ for $6 \mathrm{~h}$. Glass coverslips were coated with $0.01 \%$ poly-Llysine (PLL) for $1 \mathrm{~h}$ at $37^{\circ} \mathrm{C}$ and washed twice with PBS for $5 \mathrm{~min}$ for microglia culture. Growth of microglia in plastic plates did not require any coating. The cultures were maintained under standard cell culture conditions at $37^{\circ} \mathrm{C}$ with $5 \% \mathrm{CO}_{2}$.

Table 1. The media for the cultures of primary microglia, mixed glial culture, bone marrow-derived macrophages and L929 cell line, treatment, and live cell imaging.

\begin{tabular}{lc}
\hline \multicolumn{1}{c}{ Medium } & Components \\
\hline DMEM/pyruvate medium & DMEM (Gibco $\left.{ }^{\text {TM }}, 41965\right)$ \\
& $1 \mathrm{mM}$ sodium pyruvate $(100 \mathrm{mM}$ sodium pyruvate, \\
Thermo Fisher Scientific, 11360)
\end{tabular}


Pen/strep (1:200) (penicillin-streptomycin, $\mathrm{Gibco}^{\mathrm{TM}}$, 15070063)

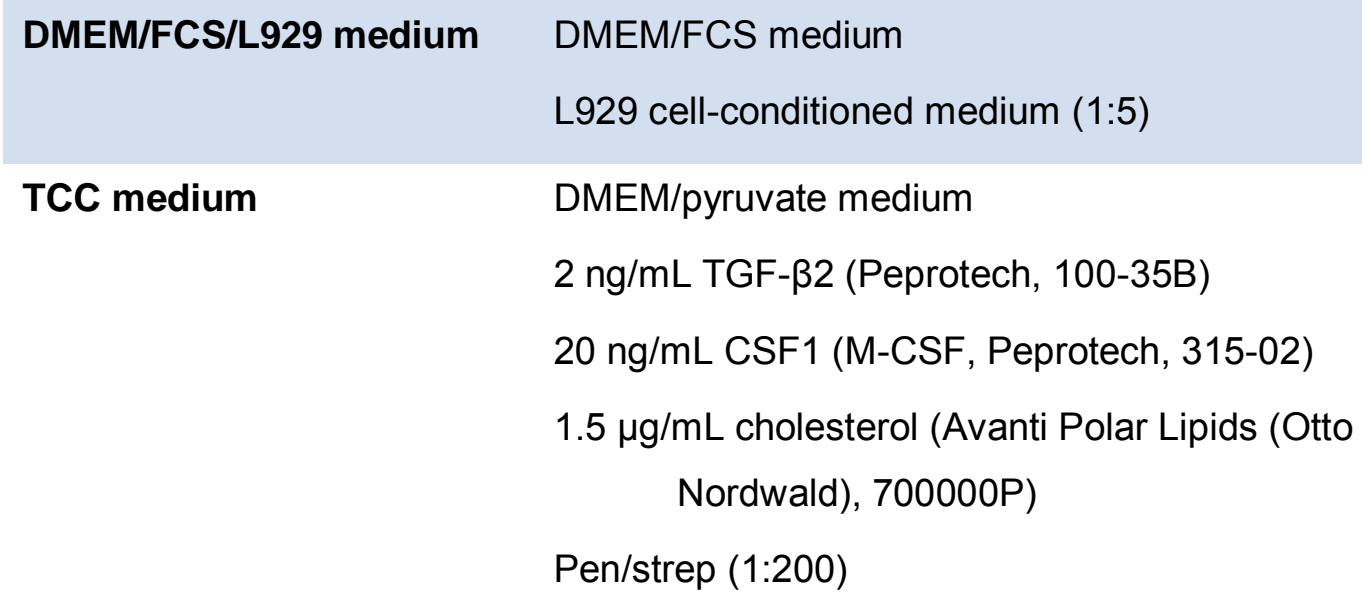




\begin{tabular}{|c|c|}
\hline & $1.5 \mu \mathrm{g} / \mathrm{mL}$ cholesterol \\
\hline & $1 \times$ GlutaMAX \\
\hline & $1 \mathrm{mM}$ sodium pyruvate \\
\hline & 20 mM HEPES (1 M HEPES, Gibco $\left.{ }^{\mathrm{TM}}, 15630056\right)$ \\
\hline $\begin{array}{l}\text { DMEM for secretome } \\
\text { analysis }\end{array}$ & $\begin{array}{l}\text { DMEM, high glucose, no glutamine, no phenol red } \\
\qquad\left(\text { Gibco }^{\mathrm{TM}}, 31053028\right)\end{array}$ \\
\hline & $1 \mathrm{mM}$ sodium pyruvate \\
\hline & 1× GlutaMAX \\
\hline
\end{tabular}

MACS microglial cell cultures were prepared from the brains of P9-P10 mouse pups, when the number of microglia was high and the dissociation of brain tissue was relatively easy. P6-P8 brains yielded fewer cells but also worked. Cell suspension was obtained by automated dissociation using the Neural Tissue Dissociation Kit (P) (Miltenyi Biotec, 130092-628) and the gentleMACS ${ }^{\mathrm{TM}}$ Dissociator (Miltenyi Biotec, 130-093-235) following the datasheet of the kit with some modifications. 2 or 3 brains were transferred to each $C$ Tube (Miltenyi Biotec, 130-096-334). DMEM/pyruvate medium was used instead of HBSS during tissue dissociation. All media were warmed up to RT. The gentleMACS programs for the brain were run twice per tube. The optional centrifugation steps were included in the protocol. For the filtering of cell suspension through $70 \mu \mathrm{m}$ cell strainers (Corning, 352350), $5 \mathrm{~mL}$ of cell suspension was applied to one cell strainer and washed with $5 \mathrm{~mL}$ of DMEM/pyruvate medium. After filtering, the cell suspension was centrifuged at $300 \times g$ for 15 or $20 \mathrm{~min}$. The cells were resuspended and topped up to $20 \mathrm{~mL}$, and the sample was diluted $1 / 10$ for counting the cell number using a hemocytometer. The cell suspension was centrifuged again at $300 \times g$ for 15 or $20 \mathrm{~min}$. The myelin removal step was omitted. Microglia were isolated by magnetic labeling with CD11b MicroBeads (Miltenyi Biotec, 130-093-634). DMEM/FCS medium instead of the buffer was used, and the serum was needed to block nonspecific binding. After the incubation with beads on ice, the cells were washed with $2 \mathrm{~mL}$ of DMEM/FCS medium per $10^{7}$ cells, and centrifuged at $300 \times g$ for 12 $15 \mathrm{~min}$ at $4^{\circ} \mathrm{C}$. The pellet was resuspended in $500 \mu \mathrm{L}$ of DMEM/FCS medium per $10^{7}$ cells. CD $11 b^{+}$cells were separated from the other cells in LS columns (Miltenyi Biotec, 130-042-401) and QuadroMACS Separator (Miltenyi Biotec). After magnetic separation, CD11 $\mathrm{b}^{+}$cells were flushed out in DMEM/FCS/L929 medium. The cells were counted, diluted to the desired density and volume in DMEM/FCS/L929 medium and seeded in multiple well plates or 8-well $\mu$-slides (Table 2). 
Table 2. Seeding of microglial cell cultures.

\begin{tabular}{|c|c|c|c|c|}
\hline Method & Plate / slide & Cell number per well & Volume of medium & Minimum volume \\
\hline \multirow{3}{*}{ MACS } & 48-well plate & $7 \times 10^{4}-1 \times 10^{5}$ & $250 \mu \mathrm{L}$ & $190 \mu \mathrm{L}$ \\
\hline & $\mu$-slide 8 well & $9 \times 10^{4}-1 \times 10^{5}$ & $300 \mu \mathrm{L}$ & $150 \mu \mathrm{L}$ \\
\hline & 6-well plate & $8 \times 10^{5}-1.2 \times 10^{6}$ & $2.5 \mathrm{~mL}$ & $1 \mathrm{~mL}$ \\
\hline \multirow{2}{*}{ Shake } & 48-well plate & $2.5 \times 10^{4}$ & $250 \mu \mathrm{L}$ & $190 \mu \mathrm{L}$ \\
\hline & 24-well plate & $5 \times 10^{4}$ & $500 \mu \mathrm{L}$ & $380 \mu \mathrm{L}$ \\
\hline
\end{tabular}

Microglial cell cultures used to be prepared by shaking mixed glial cultures. Mixed glial cultures were prepared from P1-2 NMRI or C57BL/6 mouse pups. One $75 \mathrm{~cm}^{2}$ culture flask per brain was coated with $0.01 \%$ PLL for $1 \mathrm{~h}$ at $37^{\circ} \mathrm{C}$, washed twice with PBS, and incubated with BME medium (Table 1) overnight at $37^{\circ} \mathrm{C}$. After the brains were dissected out, meninges were removed in warm Hank's Balanced Salt Solution (no calcium, no magnesium) (HBSS, Gibco). The hemispheres and midbrains were incubated with $0.25 \%$ trypsin-EDTA (Gibco, 25200) at $37^{\circ} \mathrm{C}$ for $10 \mathrm{~min}$. After trypsinization, the brains were transferred to the BME medium and triturated to get a single-cell suspension. The

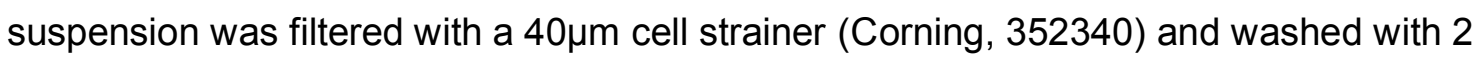
$\mathrm{mL}$ of BME medium. The cell suspension was distributed to coated $75 \mathrm{~cm}^{2}$ flasks containing $5 \mathrm{~mL}$ of BME medium and $5 \mathrm{~mL}$ of super Sato (Table 1). If the cultures were maintained for oligodendrocyte culture, the medium was exchanged and microglia were washed off until the culture had enough oligodendrocytes. After the shaking of oligodendrocytes, the cultures were incubated with DMEM/FCS/L929 medium for 6 days until microglia reached $\sim 80 \%$ confluency. The cultures were shaken at $120 \mathrm{rpm}$ at $37^{\circ} \mathrm{C}$ for 1-2 h. The cells were collected by centrifugation at $800 \mathrm{rpm}$ (Centrifuge 5702R, Eppendorf) for $10 \mathrm{~min}$ at RT. The pellet was resuspended in DMEM/FCS medium. Microglia were seeded in multiple well plates or dishes at the desired density (Table 2).

Bone marrow-derived macrophages (BMDM) were prepared as described before (CantutiCastelvetri et al., 2018). The femur and tibia of adult C57BL/6 and $M y d 88^{-/-}$mice were isolated and cut at both ends. The bone marrow was flushed out with DMEM using a syringe. A single-cell suspension was made from bone marrow by pipetting and was filtered by a cell strainer. In 24-well plates, $4.5 \times 10^{4}$ cells in $1 \mathrm{~mL}$ of DMEM/FCS/L929 medium were seeded per well. The macrophage cultures were prepared by Dr. Ludovico Cantuti-Castelvetri. The cells were washed and the DMEM/FCS/L929 medium was 
renewed every 2 or 3 days. After 4 days in vitro (DIV), the macrophage cultures were washed with DMEM and treated with the reagent in DMEM/M-CSF (Table 1).

The conditioned medium of L929 cell line contains factors such as M-CSF that stimulate the growth of microglia and macrophages. L929 cells were cultured in DMEM/FCS medium. The medium was renewed every 2 or 3 days. L929 cells were split at a subcultivation ration of $1: 2$ to $1: 8$ three times to obtain cultures in $10175 \mathrm{~cm}^{2}$ flasks. The medium was conditioned with confluent L929 cultures for 10-14 days. L929 cellconditioned medium was collected and passed through a $0.22 \mu \mathrm{m}$ filter. The aliquots were stored at $-20^{\circ} \mathrm{C}$.

\section{Live cell imaging}

Microglia were isolated by MACS and seeded in PLL-coated $\mu$-slides 8 well ibiTreat (ibidi, 80826) for live cell imaging (Table 2). The cells were cultured in DMEM/FCS/L929 medium to $90 \%$ confluency for 4-7 days, washed twice with DMEM/pyruvate to remove residual serum, and then incubated with serum-free TCC medium overnight (Table 1). The TCC/HEPES/FluoroBrite medium (Table 1) was used for live cell imaging experiments. FluoroBrite $\mathrm{DMEM}^{\mathrm{TM}}$ is a clear medium with low background fluorescence. The medium was supplemented with 20 mM HEPES to maintain the $\mathrm{pH} 7.4$ (as in the Live Cell Imaging Solution, Invitrogen ${ }^{\mathrm{TM}}$, A14291DJ). Before treatment, the cell membrane and nuclei were pre-labeled by the incubation with $1 \mu \mathrm{g} / \mathrm{mL}$ cholera toxin subunit $B(C T-B)$ Alexa Fluor ${ }^{\mathrm{TM}} 647$ conjugate (Invitrogen ${ }^{\mathrm{TM}}$, C34778) and $10 \mu \mathrm{g} / \mathrm{mL}$ Hoechst 33342 (Invitrogen ${ }^{\mathrm{TM}}$, H3570) for 15min. Microglia cultures were treated with 30 $\mathrm{gg} / \mathrm{mL}$ PKH67labeled myelin (pulse) and 40 nM LysoTracker ${ }^{\mathrm{TM}}$ Red DND-99 (Invitrogen ${ }^{\mathrm{TM}}$, L7528) for $15 \mathrm{~min}$. After treatment, the medium was changed to TCC/HEPES/FluoroBrite, and the cells were incubated for $60 \mathrm{~min}$ (chase) before imaging. The images were acquired using a Leica SP5 confocal microscope. Tile scans were made with the $63 \times$ oil objective, pinhole 1 airy unit and zoom factor 1.5 . The pre-labeling of cell membrane with CT-B could minimize nonspecific binding of CT-B to myelin membrane. LysoTracker was used before the chase to preserve the signal and avoid the accumulation of excessive LysoTracker inside cells. 


\section{Organotypic hippocampal slice culture and ex vivo demyelination models}

Organotypic hippocampal slice culture (OHSC) was prepared at a thickness of $375 \mu \mathrm{m}$ using the interface method as described (Masuch et al., 2016). The cultures were prepared from the brains of $\mathrm{P} 2 \mathrm{C} 57 \mathrm{BL} / 6$ mouse pups to have the same timing of myelination in culture. Hippocampal slices were cultured on small pieces of LCR membrane filter (Merck Millipore, FHLC04700) placed on Millicell cell culture inserts (Merck Millipore, PICM03050). LCR membrane was put in a big glass dish, wet with deionized water, flattened, air-dried, and then cut into small pieces of around $5 \mathrm{~mm} \times 6$ $\mathrm{mm}$ using a scalpel. The confetti LCR membrane was autoclaved in the dish. In a 6-well plate, $0.7-1 \mathrm{~mL}$ of OHSC culture medium (Table 3 ) was pipetted in each well. Cell culture inserts were placed in the wells, and 3 or 4 pieces of confetti LCR membrane were placed carefully on each insert (bubbles should be avoided). The plates were conditioned in the cell culture incubator for at least $2 \mathrm{~h}$. Medium A was kept at $4{ }^{\circ} \mathrm{C}$. The MCILWAIN Tissue Chopper, stereomicroscope and dissection tools were disinfected with $70 \%$ ethanol and UV under a laminar hood. The brain was dissected out and transferred immediately to icecold Medium A in a 60-mm culture dish placed on ice. Under the microscope, the interhemispheric fissure was cut, and the hemispheres were separated from the midbrain with Dumont \#5 fine forceps, tilted at $\sim 45^{\circ}$ angle. The hemisphere was positioned with the inner medial site facing up. Residual midbrain or meninges were carefully removed to expose the hippocampus without distorting it. Cuts were made with forceps in the middle of the cortex along the hippocampus, leaving a piece of cortex was attached. After the hippocampus was dissected out, it was transferred to the platform of the tissue chopper with a cut transfer pipette (Thermo Fisher Scientific, 232-1S). The liquid was removed from the stage with a transfer pipette with a fine tip. The platform was rotated and fixed to place the hippocampus perpendicular to the blade. After cutting at $375 \mu \mathrm{m}$, the slices were transferred with a cut transfer pipette to a dish containing Medium A on ice. The slices with clear hippocampal structures were selected and transferred to confetti LCR membrane in the plate. OHSC was maintained at $37{ }^{\circ} \mathrm{C}$ with $5 \% \mathrm{CO}_{2}$. The culture medium was changed the day after culture preparation, and then every 2 or 3 days. After 7 DIV, OHSC recovered from the culture preparation and were ready for experiments (Masuch et al., 2016). Myelination was mostly completed after 13 DIV but continued slowly afterwards (data not shown). Therefore, demyelination models were induced in DIV13 OHSC.

The assay for the effects of molecules on myelination in OHSC was established using 3,3',5-triiodo-L-thyronine (T3) (Sigma-Aldrich, T6397) as a positive control. OHSC was treated with 40 or $80 \mathrm{ng} / \mathrm{mL}$ T3 in the culture medium from 7 DIV for 5 days. 
Table 3. Components of the OHSC culture medium and the Medium A for OHSC preparation.

\begin{tabular}{|c|c|c|}
\hline OHSC culture medium & $100 \mathrm{~mL}$ & Concentration \\
\hline $\begin{array}{l}\text { MEM (10x), no glutamine, with Earle's } \\
\text { salts (Gibco, 21430) }\end{array}$ & $5 \mathrm{~mL}$ & $50 \%$ \\
\hline BME, Earle's salts (Gibco, 41010) & $5 \mathrm{~mL}$ & $5 \%$ \\
\hline Horse serum & $25 \mathrm{~mL}$ & $25 \%$ \\
\hline $\begin{array}{l}\text { GlutaMAX'M supplement (100x) (Gibco, } \\
35050)\end{array}$ & $1 \mathrm{~mL}$ & $1 \times$ \\
\hline 45\% glucose (Sigma, G8769) & $1.44 \mathrm{~mL}$ & $0.648 \%$ \\
\hline $7.5 \% \mathrm{NaHCO}_{3}$ & $\begin{array}{l}\text { Adjust } \mathrm{pH} \text { to } 7.2 \text {, measured } \\
\text { by } \mathrm{pH} \text { test strips }\end{array}$ & \\
\hline Penicillin/streptomycin & $0.5 \mathrm{~mL}$ & \\
\hline Autoclaved deionized water & To $100 \mathrm{~mL}$ & \\
\hline
\end{tabular}

\begin{tabular}{ll|l} 
Medium A & $514 \mathrm{~mL}$ & Concentration \\
\hline HBSS 1× (Gibco, 14175) & $500 \mathrm{~mL}$ & \\
HEPES (1 M) (Gibco, 15630-080) & $7.5 \mathrm{~mL}$ & $15 \mathrm{mM}$ \\
$45 \%$ glucose & $6.5 \mathrm{~mL}$ & $0.57 \%$
\end{tabular}

Demyelination was induced by the injection of $0.5 \mathrm{mg} / \mathrm{mL}$ in DIV13 OHSC using the Nanoliter 2010 Injector with MICRO4 controller (World Precision Instruments) installed on an MM 33 micromanipulator (Science Products). The capillaries were pulled in the same way as for stereotactic injection. To identify the injection site and the region where the injected reagent diffused to, lysolecithin was labeled with $1 \mu \mathrm{M} \mathrm{PKH26} \mathrm{(PKH26} \mathrm{red}$ fluorescent cell linker mini kit, Sigma, MINI26) in PBS. To induce a small demyelinated lesion in the hippocampal slice, $50 \mathrm{~nL}$ of $0.5 \mathrm{mg} / \mathrm{mL}$ lysolecithin was injected at a speed of $0.100 \mathrm{~nL} / \mathrm{min}$ at a depth of $150 \mu \mathrm{m}$ from the surface; 1 min after injection, the capillary was retracted carefully. The injection was performed under a cell culture hood. The OHSC was kept in the incubator between injections of different slices in order to avoid drying. 
Autoimmune demyelination model in OHSC was established with anti-MOG Z2 antibody and guinea pig complement. The anti-MOG Z2 mouse antibody and humanized 8-18C5 antibody were generously provided by the Laboratory of Prof. Dr. Edgar Meinl. The first trial used $10 \mu \mathrm{g} / \mathrm{mL}$ Z2 (Elliott et al., 2012) or 8-18C5 (Linnington et al., 1984) antibody. Z2 induced more demyelination (data not shown) than 8-18C5, and thus the demyelination model using $Z 2$ was further optimized. Mouse $\lg _{2 \mathrm{aK}}$ (mlg $\mathrm{Ig}_{2 \mathrm{a}} \mathrm{K}$, Sigma, M7769) (Derfuss et al., 2009) was used as an isotype control antibody. The batch of the complement sera from guinea pig (gpC) (Sigma, S1639) had a hemolytic titer of $216 \mathrm{CH}_{50}$ units $/ \mathrm{mL}$.

Demyelination was induced by the treatment of DIV13 OHSC with $10 \mu \mathrm{g} / \mathrm{mL}$ anti-MOG Z2 antibody and $5 \%$ guinea pig complement in the culture medium for $72 \mathrm{~h}$. The depletion of microglia in OHSC was done by the treatment with $100 \mu \mathrm{g} / \mathrm{mL}$ clodronate (Calbiochem, 233183) for 5 days from 8 DIV.

\section{Immunohistochemistry}

Immunohistochemistry $(\mathrm{IHC})$ of lesions was conducted on mounted spinal cord sections due to the fragile property of lesions. To help the attachment of mounted cryosections to the slides, the slides were dried in a $37{ }^{\circ} \mathrm{C}$ incubator for $30 \mathrm{~min}$ (or at RT for at least $1 \mathrm{~h}$ ) and washed twice with PBS for $5 \mathrm{~min}$. A circle was drawn around each section with a Super PAP Pen Liquid Blocker Mini (Science Services, N71312) for the incubation with solution. For the IHC for cytoplasmic proteins (BCAS1, IBA1, galectin-3, MHC class II, GFAP, etc.), the sections were blocked and permeabilized with blocking solution containing $0.1 \%$ Triton $\mathrm{X}-100$ for $1 \mathrm{~h}$ at $\mathrm{RT}$. The sections were incubated with primary antibodies in blocking solution containing $0.1 \%$ Triton X-100 overnight at $4{ }^{\circ} \mathrm{C}$ (one section incubated without primary antibodies as a control for the specificity of secondary antibodies), and washed three times with PBS for $10 \mathrm{~min}$. The sections were then incubated with secondary antibodies in blocking solution containing $0.1 \%$ Triton X-100 for $1-2 \mathrm{~h}$ at RT, following by a wash with PBS for $10 \mathrm{~min}$. Mild permeabilization using $0.1 \%$ Triton X-100 was required for FluoroMyelin stain. After the incubation with secondary antibodies, the sections were incubated with 1:350 FluoroMyelin ${ }^{\mathrm{TM}}$ Green Fluorescent Myelin Stain (Molecular Probes ${ }^{\mathrm{TM}}$, F34651) in PBS for 20 min at RT. The nuclei were stained with $1 \mu \mathrm{g} / \mathrm{mL}$ 4',6-diamidino-2-phenylindole (DAPI) in PBS for 15-30 min at RT. The sections were washed twice with PBS for 10 min, mounted with Mowiol or ProLong ${ }^{\mathrm{TM}}$ Diamond Antifade Mountant (Thermo Fisher Scientific, P36970) and \#1.5 coverslip, and air-dried overnight. The IHC for OLIG2, NKX-2.2 and APC (CC-1) required heat-induced 
antigen retrieval in $10 \mathrm{mM}$ sodium citrate, $\mathrm{pH}$ 6.0. The sections were heated using the 120 ${ }^{\circ} \mathrm{C}$ autoclaving program for liquid. After cooling down, the sections were washed twice with PBS for $10 \mathrm{~min}$, and permeabilized with blocking solution containing $0.3 \%$ Triton X100 for $1 \mathrm{~h}$ at RT. The sections were incubated with primary antibodies blocking solution containing $0.1 \%$ Triton $\mathrm{X}-100$ for 2 days at $4{ }^{\circ} \mathrm{C}$; this longer incubation allows optimal staining of all oligodendrocyte-lineage cells with the anti-OLIG2 antibody. After washing, the sections were incubated with secondary antibodies in blocking solution containing $0.1 \%$ Triton $\mathrm{X}-100$ for $2 \mathrm{~h}$ at $\mathrm{RT}$, and the other procedures were the same as for cytoplasmic antigens. The dilutions of primary and secondary antibodies for $\mathrm{IHC}$ are listed in Table 5 and Table 6, respectively.

The IHC of cultured organotypic hippocampal slices were carried out as a free-floating protocol with the slices on confetti LCR membrane. The slices were washed three times with warm PBS, fixed with 4\% PFA in PBS for $2 \mathrm{~h}$ at RT, and washed three times with PBS for 5 min with mild shaking. For the IHC for MBP, neurofilament heavy polypeptide (NF-H) and IBA1, the slices were blocked and permeabilized with blocking solution containing $0.1 \%$ saponin and $0.02 \%$ sodium azide (block/saponin/ $\mathrm{NaN}_{3}$ ) for 1 day at RT with mild shaking. To stain most part of the thick slices, the slices were incubated with primary antibodies in block/saponin/ $\mathrm{NaN}_{3}$ for 7 days at $37^{\circ} \mathrm{C}$ with shaking. The slices were washed six times with PBS for $30 \mathrm{~min}$ at RT, incubated with secondary antibodies in block/saponin/ $\mathrm{NaN}_{3}$ for 1 day at $37^{\circ} \mathrm{C}$, and then washed three times with PBS for 30 min at RT. The nuclei were stained with $1 \mu \mathrm{g} / \mathrm{mL}$ DAPI in PBS for $30 \mathrm{~min}$ at RT. After two washes with PBS for $30 \mathrm{~min}$, the slices on confetti LCR membrane were mounted on SuperFrost Plus slides with mounting medium and \#1.5 coverslip (Figure 14D and E, and Figure 15). The IHC protocol was modified from the whole-mount immunostaining method (Belle et al., 2014). On the other hand, the staining for myelin debris (MBP) inside microglia (IBA1) after the injection of lysolecithin in OHSC was done at $4{ }^{\circ} \mathrm{C}$ without excessive washing. After fixation, the slices were blocked ant permeabilized with blocking solution containing $0.1 \%$ Triton $\mathrm{X}-100$ for 1 day at $4{ }^{\circ} \mathrm{C}$ with mild shaking, and then incubated with primary antibodies (anti-MBP mouse and anti-IBA1 rabbit antibodies) in blocking solution containing $0.1 \%$ Triton $\mathrm{X}-100$ for 6 days at $4{ }^{\circ} \mathrm{C}$. After three washes with PBS for 10 min at RT, the slices were incubated with secondary antibodies in blocking solution containing $0.1 \%$ Triton X-100 overnight at $4{ }^{\circ} \mathrm{C}$. The slices were then washed with PBS for 10 min at RT, incubated with $1 \mu \mathrm{g} / \mathrm{mL}$ DAPI for $15 \mathrm{~min}$, washed twice with PBS for 10 min and mounted (Figure 14B and C). 
Table 4. Buffers and solutions for immunohistochemistry and immunocytochemistry.

\begin{tabular}{|c|c|}
\hline Solution & Components \\
\hline \multirow[t]{5}{*}{ PBS (pH 7.4) } & $137 \mathrm{mM} \mathrm{NaCl}$ \\
\hline & $2.7 \mathrm{mM} \mathrm{KCl}$ \\
\hline & $10 \mathrm{mM} \mathrm{Na}_{2} \mathrm{HPO}_{4}$ \\
\hline & $1.8 \mathrm{mM} \mathrm{KH}_{2} \mathrm{PO}_{4}$ \\
\hline & Deionized water \\
\hline \multirow[t]{2}{*}{ Citrate buffer (pH 6.0) } & $10 \mathrm{mM}$ sodium citrate \\
\hline & Deionized water \\
\hline \multirow[t]{4}{*}{ Blocking solution } & $2.5 \%$ bovine serum \\
\hline & $2.5 \%$ BSA \\
\hline & $2.5 \%$ fish gelatin (Sigma, G7765) \\
\hline & PBS \\
\hline \multirow[t]{4}{*}{$10 \%$ blocking solution } & $1 \%$ bovine serum \\
\hline & $1 \% \mathrm{BSA}$ \\
\hline & $1 \%$ fish gelatin \\
\hline & PBS \\
\hline \multirow[t]{3}{*}{ Block/saponin/ $\mathrm{NaN}_{3}$} & Blocking solution \\
\hline & $0.1 \%$ saponin (Sigma, S4521) \\
\hline & $0.02 \%$ sodium azide \\
\hline
\end{tabular}

Table 5. Primary antibodies used in IHC.

\begin{tabular}{llll}
\hline \multicolumn{1}{c}{ Antibody } & Dilutio & \multicolumn{1}{c}{ Notes } \\
& $\mathbf{n}$ & \\
\hline $\begin{array}{l}\text { Anti-APC (Ab-7) mouse mAb (CC-1) (Calbiochem }{ }^{\circ}, \\
\text { OP80) }\end{array}$ & $1: 100$ & Antigen retrieval \\
Anti-Bcas1 rabbit polyclonal antibody & $1: 300$ & Mild \\
& & permeabilization \\
$\begin{array}{l}\text { Purified anti-mouse/human Mac-2 (galectin-3) rat } \lg _{2 \mathrm{a}} \mathrm{K} \\
\text { (BioLegend, 125401) }\end{array}$ & & \\
\hline
\end{tabular}


Anti-GFAP polyclonal guinea pig antiserum (SYSY, $1: 500$

173004)

Anti IBA1 polyclonal antibodies, rabbit (Wako, 019-

1:500 Mild

19741)

permeabilization

Anti-myelin basic protein rat monoclonal $\operatorname{lgG}_{2 a}$ antibody $\quad 1: 100 \quad 0.1 \%$ saponin (Abcam, ab7349)

Purified anti-myelin basic protein, clone SMI 94, mouse $\quad 1: 500$

$\lg _{1} \mathrm{~K}$ (BioLegend, 836504)

Anti-mouse MHC class II (I-A/I-E) purified rat lgG2b k 1:100 Mild

(Affymetrix eBioscience, 14-5321)

permeabilization

Anti-neurofilament heavy polypeptide chicken polyclonal $\quad 1: 1000$

$\lg Y$ antibody (Abcam, ab4680)

Nkx2.2 mouse hybridoma, clone 74.5A5 (DSHB, U lowa) 1:5 Antigen retrieval

Anti-Olig-2 rabbit polyclonal antibody (Chemicon ${ }, \quad 1: 250 \quad$ Antigen retrieval AB9610)

Table 6. Secondary antibodies used in IHC.

\begin{tabular}{|c|c|}
\hline Antibody & Dilution \\
\hline Goat anti-rabbit lgG $(\mathrm{H}+\mathrm{L})$ cross-adsorbed secondary antibody, Alexa & $1: 1000-$ \\
\hline Fluor 647 (Thermo Fisher Scientific, A-21244) & $1: 750$ \\
\hline $\begin{array}{l}\text { Goat anti-rabbit lgG }(\mathrm{H}+\mathrm{L}) \text { cross-adsorbed secondary antibody, Alexa } \\
\text { Fluor } 555 \text { (Thermo Fisher Scientific, A-21428) }\end{array}$ & $1: 1000$ \\
\hline $\begin{array}{l}\text { Goat anti-rabbit lgG }(\mathrm{H}+\mathrm{L}) \text { cross-adsorbed secondary antibody, Alexa } \\
\text { Fluor } 488 \text { (Thermo Fisher Scientific, A-11008) }\end{array}$ & $1: 1000$ \\
\hline $\begin{array}{l}\text { Goat anti-rat IgG }(H+L) \text { cross-adsorbed secondary antibody, Alexa Fluor } \\
488 \text { (Thermo Fisher Scientific, A-11006) }\end{array}$ & $\begin{array}{l}1: 1000- \\
1: 750\end{array}$ \\
\hline $\begin{array}{l}\text { Goat anti-mouse IgG }(\mathrm{H}+\mathrm{L}) \text { cross-adsorbed secondary antibody, Alexa } \\
\text { Fluor } 647 \text { (Thermo Fisher Scientific, A-21235) }\end{array}$ & $1: 1000$ \\
\hline Goat anti-mouse IgG $(\mathrm{H}+\mathrm{L})$ cross-adsorbed secondary antibody, Alexa & $1: 1000-$ \\
\hline Fluor 555 (Thermo Fisher Scientific, A-21422) & $1: 750$ \\
\hline
\end{tabular}


Goat anti-chicken $\lg \mathrm{Y}(\mathrm{H}+\mathrm{L})$ secondary antibody, Alexa Fluor 647 (Thermo 1:1000

Fisher Scientific, A-21449)

Goat anti-guinea pig secondary antibody, Cy3

$1: 500$

\section{Immunocytochemistry}

The cells cultured on coverslips were washed with warm PBS, fixed with 4\% PFA in PBS for 10-15 min at RT, and washed three times with PBS for $5 \mathrm{~min}$. To analyze the amount of $\mathrm{PKH}$-labeled myelin or non-myelin membrane in microglia, the cells were blocked with blocking solution for $30 \mathrm{~min}$ without permeabilization. The cell membrane was stained with lectin in 10\% blocking solution for $45 \mathrm{~min}$, and washed with PBS for $5 \mathrm{~min}$. After the counterstain with $1 \mu \mathrm{g} / \mathrm{mL}$ DAPI in PBS for $15 \mathrm{~min}$ at RT, the coverslips were washed twice with PBS and once with distilled water, dried quickly and mounted on slides (Figure $4 \mathrm{~A}-\mathrm{C}$ ). The immunocytochemistry (ICC) for cell surface antigens galectin-3 and $\mathrm{MHC}$ class II was also done without permeabilization. After blocking, the cells were incubated with primary antibody in $10 \%$ blocking solution for $1 \mathrm{~h}$, and washed three times with PBS for $5 \mathrm{~min}$. The cells were incubated with secondary antibody in 10\% blocking solution for 45 min-1 h (Figure S1A and B). After microglia were treated with PKH67-labeled myelin, the fixed cells were stained for LAMP-2 with the anti-CD107b primary antibody. Blocking and permeabilization, and the incubations with primary antibody and secondary antibody (and lectin) were carried out in blocking solution containing $0.05 \%$ saponin (Figure 7). Saponin allows mild permeabilization by removing cholesterol in the cell membrane (Scheffler et al., 2014). The ICC for iNOS of microglia after the treatment with myelinPKH67 was also done with $0.05 \%$ saponin for mild permeabilization (Figure S1C).

Table 7. Primary antibodies used in ICC.

\begin{tabular}{|c|c|c|}
\hline Antibody & Dilution & Notes \\
\hline $\begin{array}{l}\text { Purified anti-mouse/human Mac-2 (galectin-3) rat } \lg G_{2 a} K \\
\text { (BioLegend, 125401) }\end{array}$ & $1: 250$ & $\begin{array}{l}\text { No } \\
\text { permeabilization }\end{array}$ \\
\hline $\begin{array}{l}\text { Anti-mouse MHC class II (I-A/I-E) purified rat } \lg _{2 b} \mathrm{~K} \\
\text { (Affymetrix eBioscience, 14-5321) }\end{array}$ & $1: 200$ & $\begin{array}{l}\text { No } \\
\text { permeabilization }\end{array}$ \\
\hline $\begin{array}{l}\text { Purified rat anti-mouse CD107b (LAMP-2), clone M3/84, } \\
\operatorname{lgG}_{1} \mathrm{~K} \text { (BD Pharmingen, 553322, 0.5mg/mL) }\end{array}$ & 1:1000 & $0.05 \%$ saponin \\
\hline
\end{tabular}


Anti-iNOS/NOS II rabbit polyclonal antibody (Merck

Millipore, ABN26)

Anti-NF-kB p65 rabbit polyclonal IgG antibody (Abcam, $\quad 1: 100$ ab16502) 
Table 8. Secondary antibodies and dyes used in ICC.

\section{Antibody / dye}

\section{Dilution}

DyLight 649, 594 or 488-labeled Lacopersicon esculentum (tomato) lectin $\quad 1: 200$ (Vector Laboratories DL-1178, 1177 or 1174$)(1 \mathrm{mg} / \mathrm{mL})$ $(5 \mu \mathrm{g} / \mathrm{mL})$

Alexa Fluor® 647 AffiniPure donkey anti-rat lgG $(\mathrm{H}+\mathrm{L})$ (Jackson $1: 750$ ImmunoResearch, 712-605-150)

Goat anti-rat $\lg G(\mathrm{H}+\mathrm{L})$ cross-adsorbed secondary antibody, Alexa Fluor 1:750 555 (Thermo Fisher Scientific, A-21434)

Goat anti-rabbit $\lg G(\mathrm{H}+\mathrm{L})$ cross-adsorbed secondary antibody, Alexa $1: 1000-$

Fluor 647 (Thermo Fisher Scientific, A-21244)

\section{Image acquisition}

The fluorescent images of spinal cord lesions were acquired using the Leica DMI6000 widefield microscope using the $20 \times$ objective as tile scans (Figure 1-Figure 3 ). The images of ICC were acquired using the Leica DMI6000 widefield microscope using the 20x objective (Figure 4; Figure 7C-F; Figure S1A and B), or the Leica SP5 confocal microscope using the $20 \times$ objective (Figure S1C) or the $63 \times$ oil objective (tile scans and Figure 7 z-stack images). The images of OHSC were acquired using the Leica SP5 confocal microscope using the $20 \times$ objective as tile scans of $z$-stacks (Figure 13-Figure 15). Images were taken at least $10 \mu \mathrm{m}$ from the surface of the slices. The images of brain sections were acquired using the Leica SP8 confocal microscope using the $20 \times$ objective as tile scans (Figure 16).

The bright-field images of LFB(-PAS) stain of spinal cord sections were acquired as tile scans using the Zeiss Axiophot microscope, the Olympus BX61VS light microscope, or the Zeiss Axio Imager M2 using the $20 \times$ objective.

The bright-field images of semithin sections were acquired as tile scans using the Leica DMI6000 widefield microscope using the $40 \times$ objective by Dr. Ludovico CantutiCastelvetri. 


\section{Image analysis}

The images were analyzed using the software Fiji (Schindelin et al., 2012). Automated cell counting and measurement were conducted using CellProfiler (Carpenter et al., 2006). In CellProfiler, the nuclei were identified as primary objects based on the staining with DAPI or Hoechst 33342, according to the user-defined parameters such as thresholding method, threshold, and the diameter of objects and so on. Based on the nuclei, the cells were identified as secondary objects based on the staining for a cytoplasmic epitope or lectin stain. The staining for transcription factors such as OLIG2 was also used to identify primary objects. The objects were related and filtered, and the number of objects positive or negative for a marker was counted automatically. The regions of interest (ROI) tissue sections were masked on the images (Figure 1B-G, Figure 2, Figure 3 and Figure 16). Myelin debris and LysoTracker were also identified as primary objects and related to each other (Figure 6). The myelin debris (or LysoTracker) in each cell were identified by relating the primary objects to the cells and merging the objects that corresponded to the same cell (Figure 4 and Figure 6). The results were saved as images or exported to spreadsheets (in csv format). The numbers of remyelinated axons in the images of semithin sections were counted manually using the Fiji plugin "Cell Counter" (Figure 1H). The intensity of the bands in western blot was measured using Fiji (Figure 4F).

The myelination in OHSC was analyzed as the proportion of the signal of the staining for both MBP and neurofilament heavy polypeptide (NF-H) among the signal of NF-H, using the ImageJ macros written by Dr. loannis Alexopoulos. To analyze the area of myelin sheaths, the z-stacks were projected by maximum intensity projection of the same number of optical slices. The projection was thresholded. The $\mathrm{MBP}^{+}$fibers were selected for their relatively big size and small circularity using "Analyze Particles", and the total area of $\mathrm{MBP}^{+}$fibers in each projection was measured (Figure 13). To analyze the percentage volume of myelination, MBP and NF-H signals were identified by thresholding the $\mathrm{z}-$ stacks. The voxels occupied by both MBP and NF-H were identified from the thresholded z-stacks using the image calculator (Figure 15). The myelination index was calculated using the following formula:

$$
\frac{V_{\mathrm{MBP}}{ }_{\mathrm{NF}-\mathrm{H}^{+}}}{V_{\mathrm{NF}-\mathrm{H}^{+}}} \times 100 \%
$$




\section{Statistics}

The data were plotted and statistical tests were performed using the software Graphpad Prism (Figure 1, Figure 2, Figure 3, Figure 4G, Figure 13, Figure 15 and Figure 16). The csv spreadsheets containing the data of individual cells were analyzed using $R$ and RStudio. The data were visualized using the package ggplot2 (Figure 4D, E and Figure 6).

\section{Laser microdissection for RNA-seq samples}

For RNA samples, all tools were cleaned and sprayed with RNaseZAP TM (Sigma, R2020). The mice were anesthetized in a $\mathrm{CO}_{2}$ chamber and euthanized by decapitation. The segment of spinal cord that contained lesions was dissected out, embedded in O.C.T. in a mold and flash-frozen in liquid nitrogen. The spinal cords were stored in a box with desiccant (Aldrich, Z163570-100EA) at the $-80^{\circ} \mathrm{C}$.

The spinal cords were placed in the cryostat for at least $30 \mathrm{~min}$ to equilibrate to $-20{ }^{\circ} \mathrm{C}$. Coronal sections were cut at a thickness of $16 \mu \mathrm{m}$ and mounted onto pre-cooled MembraneSlide 1.0 PEN (D) Zeiss (Fisher Scientific, 415190-9041-000). O.C.T. was trimmed as much as possible. The bottom of the tissue block was trimmed to be parallel to the blade. If there were cracks in the O.C.T. of the sections, the temperature was raised to -19 or $-18^{\circ} \mathrm{C}$. Several sections were cut and laid on the metal stage. The backside of the slide was warmed with fingers (wearing gloves) to warm the region for placing the sections. Then, the frozen sections were transferred to the slide by touching the warmed region. The slides with sections were dried in the cryostat for $10 \mathrm{~min}$ at $-20{ }^{\circ} \mathrm{C}$. After cryosectioning of the tissue block, the exposed tissue was covered with a drop of O.C.T. The tissue was wrapped in aluminum foil and stored with desiccant at $-80^{\circ} \mathrm{C}$. The ethanol solutions for fixation and dehydration were prepared from ethanol for molecular biology containing molecular sieves (Carl Roth, 2405886) once the bottle was opened (to ensure that the ethanol did not get diluted by moisture) and nuclease-free water. Fresh $100 \%$ ethanol was used. The solutions were pre-cooled at $-20^{\circ} \mathrm{C}$ and re-used for no more than one week. Under the fume hood for RNA, the sections were fixed and dehydrated in $70 \%$ ethanol for $30 \mathrm{sec}, 95 \%$ ethanol for $30 \mathrm{sec}$, and then twice in $100 \%$ ethanol for $1 \mathrm{~min}$ each. The slides were air-dried for $10 \mathrm{~min}$. The dried slides were used for laser microdissection, or stored in a $50-\mathrm{mL}$ conical tube with desiccant at $-80{ }^{\circ} \mathrm{C}$ for up to 1 month without affecting the quality of RNA (Espina et al., 2006; Nichterwitz et al., 2016). 
When the slides were taken out of the $-80^{\circ} \mathrm{C}$ freezer for laser microdissection, the slides were dehydrated by passing them through the same series of $-20^{\circ} \mathrm{C}$ ethanol solutions, air-dried and kept in a closed conical tube with desiccant at RT. The 0.5-mL Axygen PCR tubes with adhesive caps (VWR, 732-0675) were used to collect dissected tissue on the Leica DM6000 B laser microdissection microscope (Leica Microsystems). Microdissection was carried out using the $10 \times$ objective, laser power 33 , speed 8 , current $100 \%$ and pulse frequency 146 . The laser was calibrated on the section before microdissection. From each spinal cord, 20-45 pieces of lesions were collected in the cap of a PCR tube. The dissected tissue was checked under a stereoscope. Under the hood for RNA, $10 \mu \mathrm{L}$ of 2mercaptoethanol ( $\beta-M E$ ) (Sigma, $63689-25 \mathrm{ML}-\mathrm{F}$ ) was added to $1 \mathrm{~mL}$ of Buffer RLT (RNeasy Micro Kit (50), Qiagen, 74004). $350 \mu \mathrm{L}$ of Buffer RLT containing $\beta-M E$ was added to the tube. The cap was closed and incubated upside-down for $30 \mathrm{~min}$ at RT. After lysis, the tube was centrifuged for $5 \mathrm{~min}$ at $3000 \times \mathrm{g}$. The samples for RNA-seq were stored at $-80^{\circ} \mathrm{C}$.

\section{Transcriptomics of demyelinated lesions}

cDNA library preparation and next-generation sequencing were performed in collaboration with the Laboratory of Prof. Dr. Moritz Rossner. Differential gene expression analysis and pathway analysis of the transcriptomic data were done by Nirmal Raman Kannaiyan.

\section{Proteomics and pathway analysis of cultured microglia}

For secretome analysis, microglia isolated by MACS from WT and Myd88 ${ }^{-/}$mouse pups were seeded in 6-well plates and cultivated in DMEM/FCS/L929 medium for 5 days until the cells were confluent. The microglia cultures were washed twice with warm DMEM/pyruvate medium and incubated with serum-free TCC medium for $3 \mathrm{~h}$. The cells were treated with $30 \mu \mathrm{g} / \mathrm{mL}$ myelin debris (or HEPES control) in $1 \mathrm{~mL}$ of TCC medium for $4 \mathrm{~h}$, washed three times with warm PBS, and incubated with the DMEM for secretome analysis for $16 \mathrm{~h}$ (Table 1). The supernatant was passed through $0.22-\mu \mathrm{m}$ filters and snap-frozen in liquid nitrogen. The samples were prepared in 5 replicates. 
The cell lysate samples of microglia for the proteomic data included in this dissertation were prepared as follows. Microglia isolated by MACS from WT and Myd88/- mouse pups were seeded at a density of $1 \times 10^{6}$ cells per $60-\mathrm{mm}$ dish in DMEM/FCS/L929 medium. After 2 DIV, microglia were washed with warm DMEM/ pyruvate medium and treated with $20 \mu \mathrm{g} / \mathrm{mL}$ myelin debris (or HEPES control) in $2 \mathrm{~mL}$ of TIC medium for $4 \mathrm{~h}$. After treatment, the cells were washed with DMEM/ pyruvate medium, and incubated with $4 \mathrm{~mL}$ of DMEM containing $0.2 \%$ BSA for $16 \mathrm{~h}$. The cells in each dish were washed with $2 \mathrm{~mL}$ of cold PBS on ice, and lysed with $200 \mu \mathrm{L}$ of cell lysis buffer (Cell Signaling Technology, 9803) containing $1 \mathrm{mM}$ PMSF for $5 \mathrm{~min}$ on ice. The cells were detached using cell scapers, collected and stored at $-80^{\circ} \mathrm{C}$. The samples were prepared in triplicates or quadruplicates.

The mass spectrometry label-free quantification (LFQ) was performed in collaboration with the Laboratory of Prof. Dr. Stefan Lichtenthaler. The proteomic analysis using MaxQuant and Ingenuity Pathway Analysis were conducted by Dr. Stephan Müller. The comparisons of the following groups were analyzed:

\begin{tabular}{|c|c|c|}
\hline Comparison & Label & Ingenuity Pathway Analysis \\
\hline Myelin_WT vs. control_WT & MY_WT/CON_WT & Yes \\
\hline 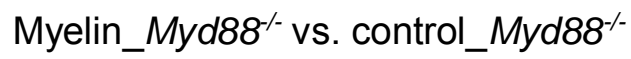 & MY_KO/CON_KO & Yes \\
\hline 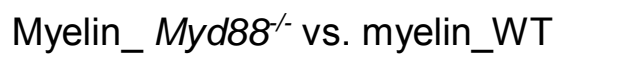 & MY_KO/MY_WT & Yes \\
\hline Control_Myd88 ${ }^{--}$vs. control_WT & CON_KO/CON_WT & - \\
\hline
\end{tabular}

The genes that fulfilled the criteria $\mid \log _{2}$ (LFQ ratio) $\mid>0.5$ and $p$-value $<0.05$ were considered as differentially expressed. The genes identified in the differential gene expression analysis were selected from the intersection of the comparisons "Myelin_WT vs. control_WT" and "Myelin_Myd88\% vs. myelin_WT" (Figure 9). The datasets of genes for which the $p$-value could be calculated were uploaded to the Ingenuity Pathway Analysis and selected for the cutoff mentioned above. For the analysis using the gene ontology enrichment analysis and visualization tool (GOrilla), two unranked lists - one of differentially expressed genes, and the other background list of the genes with a numeric $p$-value - were uploaded for pathway analysis. 


\section{Results}

\section{Remyelination was impaired in the spinal cord of $M y d 88^{-/-}$mice}

The numbers of oligodendrocytes were analyzed in the demyelinated lesions at 7 and 21 days post-injection (DPI). The lesions were identified by the lack of luxol fast blue (LFB) stain in the white matter. The lesions were filled with immune cells at $7 \mathrm{DPI}$ and appeared pink due to the periodic acid-Schiff (PAS) stain (Figure 1A). The nuclei of oligodendrocyte progenitor cells (OPCs) were identified by the co-labeling of the transcription factors NKX2.2 and OLIG2 (Figure 1B). Myelinating oligodendrocytes expressed the marker BCAS1 in their cytoplasm (Figure 1C). Mature oligodendrocytes expressed the transcription factor OLIG2 and the cytosolic marker APC (CC-1) (Figure 1D). The lesions of wild-type (WT) mice had a high number of OPCs at $7 \mathrm{DPI}$, which was decreased at $21 \mathrm{DPI}$. In contrast, the number of OPCs was low in the lesions of Myd88/- mice at 7 DPI (Figure 1E). Consistently, the lesions of WT mice had many myelinating oligodendrocytes at 7 DPI but not a $21 \mathrm{DPI}$, but the increase in myelinating oligodendrocytes at 7 DPI was not observed in the lesions of $M y d 88^{-/}$mice (Figure 1F). Mature oligodendrocytes were present in high numbers in the lesions of WT mice at $21 \mathrm{DPI}$, but the lesions of $M y d 88^{-/-}$mice were deficient in mature oligodendrocytes (Figure 1G).

Remyelination was examined in the semithin sections of the spinal cord lesions at $21 \mathrm{DPI}$. Myelin was stained by methylene blue-azure II (Figure 1K). Regenerated myelin in the lesions was also stained but thinner than the myelin in normal white matter (Figure 11 and J). While the lesions of WT mice had a variable density of remyelinated axons, the lesions of $M y d 88^{-/}$mice had very few remyelinated axons at $21 \mathrm{DPI}$ (Figure $1 \mathrm{H}$ ). Therefore, remyelination was defective in $M y d 88^{-/}$mice.

Figure 1. Remyelination was defective in the spinal cord of $M y d 88^{-}$mice.

(A) Demyelinated lesions were identified by LFB-PAS stain. Oligodendrocytes at various developmental stages were analyzed in lesions. (B) OPCs express NKX-2.2 (magenta) and OLIG2 (green). (C) Myelinating oligodendrocytes express BCAS1 (green). (D) Mature oligodendrocytes express APC (CC-1) (magenta) and OLIG2 (green). (E) Decreased cell density of OPCs in the lesions of $M y d 88^{-}$mice at 7 days post-injection (DPI). Unpaired $t$-test with FDR correction. (F) Few myelinating oligodendrocytes in the lesions of $M y d 88^{-}$mice at 7 DPI. Welch's $t$-test. (G)

Decreased number of mature oligodendrocytes in the lesions of $M y d 88^{-/}$mice at $21 \mathrm{DPI}$. Unpaired $t$-test with FDR correction. (Error bars: $95 \% \mathrm{Cl} ; n=3-10$ lesions) 
(A)

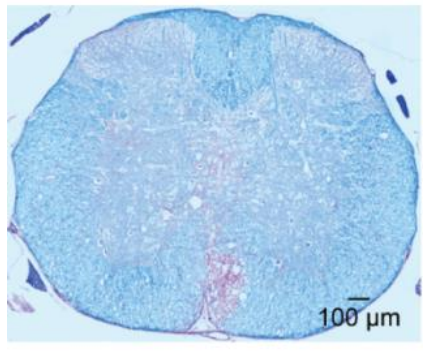

(E)

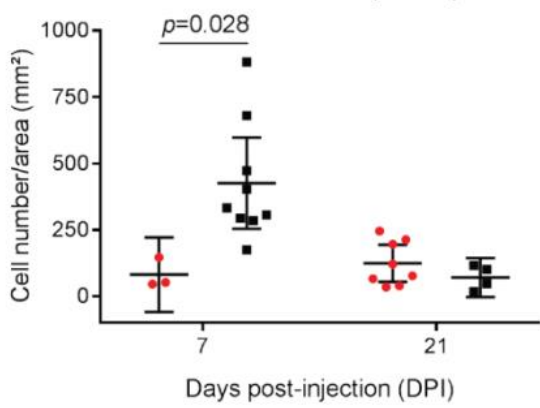

(G)

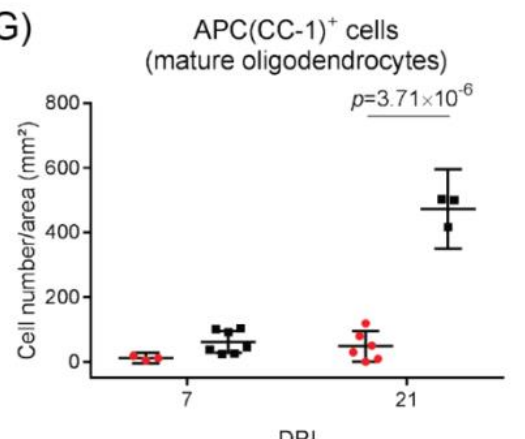

(I) $M y d 88^{-1-}$

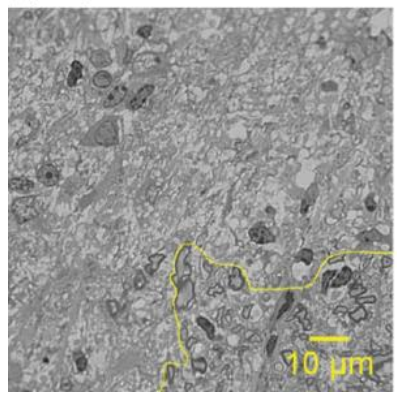

(B) NKX-2.2 OLIG2

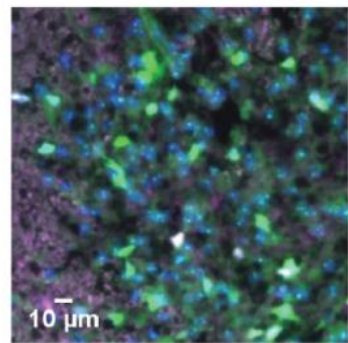

(C) BCAS1 DAPI

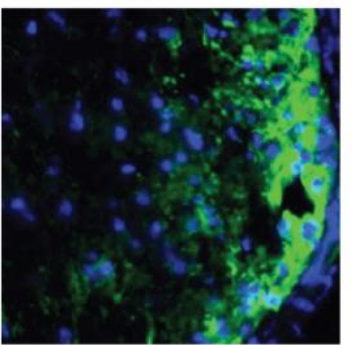

(D) CC-1 OLIG2

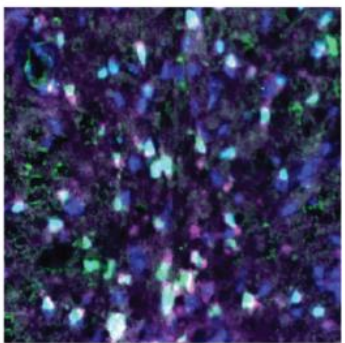

$(\mathrm{F})$

- Myd88

- WT

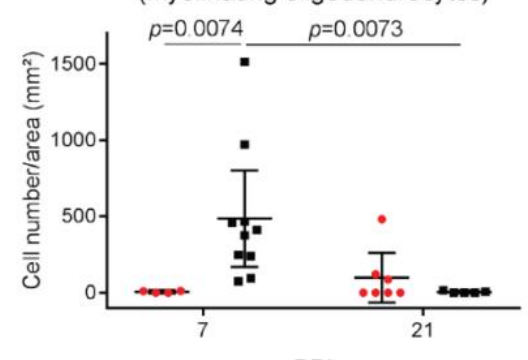

DPI
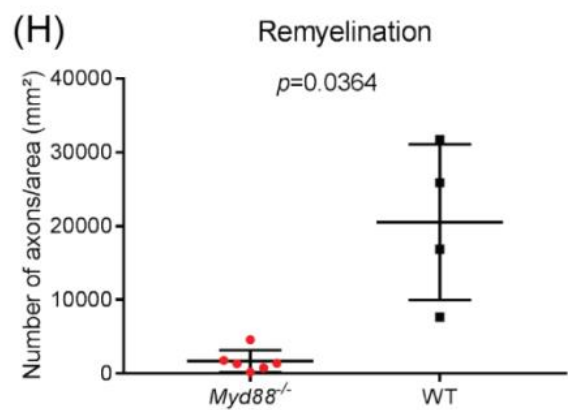

(K) normal white matter

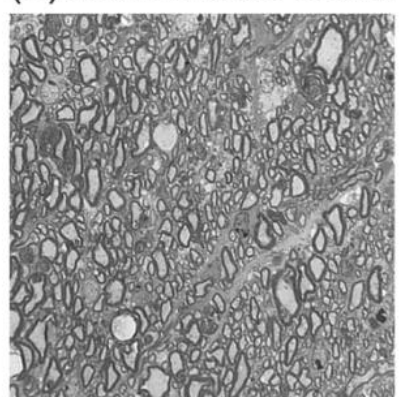

(Figure 1 continued)

(H) Impaired remyelination in the lesions of $\mathrm{Myd}^{-1 /}$ mice at $21 \mathrm{DPI}$ quantified by the density of remyelinated axons in semi-thin sections. Welch's $t$-test (error bars: SD; $n=4-6$ lesions). Lesions in (I) Myd88- and (J) WT mice. Remyelinated axons are wrapped by thin myelin (arrow heads). (K) Normal white matter is full of axons wrapped by thick myelin. (Images of semi-thin sections: courtesy of Dr. Ludovico Cantuti-Castelvetri) 


\section{Microglia / macrophages were recruited to demyelinated lesions}

The cell density of microglia / macrophages was analyzed in the core of the lesions at various time points. The cells expressing the general microglia / macrophage marker IBA1 were recruited to the lesions of both $M y d 88^{-/}$and WT mice. The density of IBA ${ }^{+}$cells peaked at $7 \mathrm{DPI}$ and decreased afterwards. At $14 \mathrm{DPI}$, more IBA $1^{+}$cells remained in the lesions of $M y d 88^{--}$mice (Figure $2 \mathrm{~A}$ and B). The FluoroMyelin Green stain inside IBA1 ${ }^{+}$ cells showed that more myelin lipids accumulated in microglia / macrophages in the lesions of $\mathrm{Myd}^{-1 /}$ than WT mice at $14 \mathrm{DPI}$ (Figure 2A and C). On the other hand, IBA ${ }^{+}$ cells contained similar amounts of myelin basic protein (MBP) in the lesions of $M y d 88^{-1-}$ than WT mice at all the time points analyzed (Figure 2D).

(A) IBA1 FluoroMyelin Green
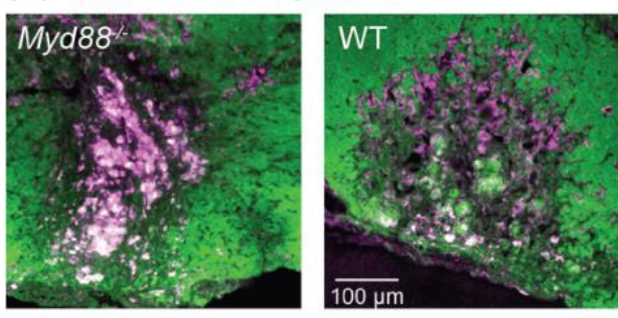

(C)

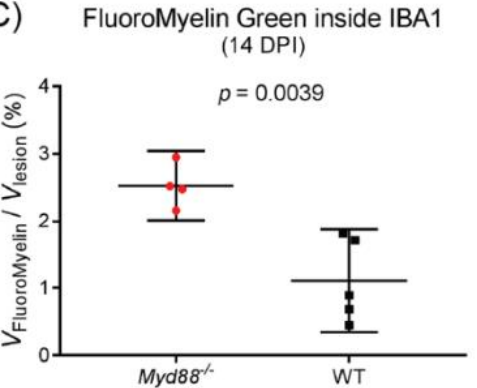

(B) IBA1 in lesions
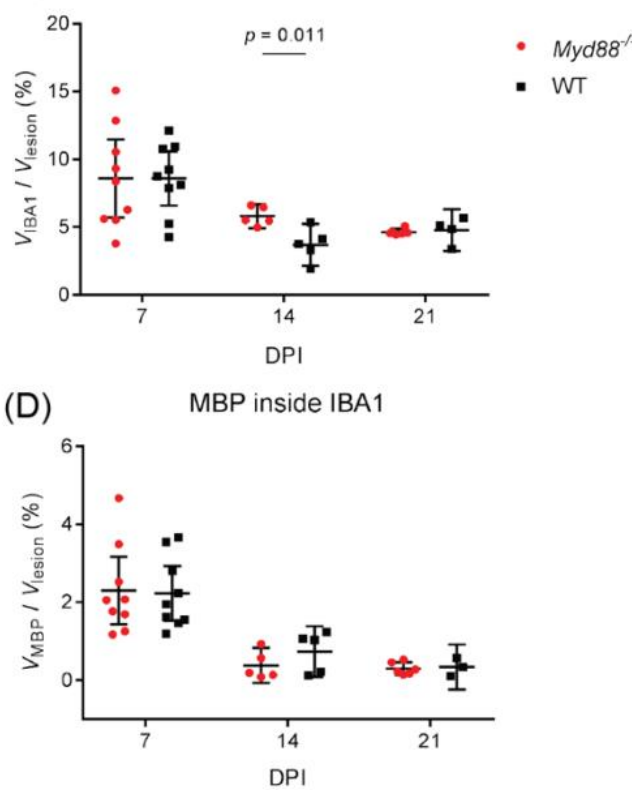

Figure 2. Microglia / macrophages containing myelin lipids remained in the lesions of $M y d 88^{-/}$mice at $14 \mathrm{DPI}$. (A) and (B) IBA1 ${ }^{+}$microglia / macrophages (magenta) were recruited to the lesions of both WT and $\mathrm{Myd} 88^{-/}$mice at $7 \mathrm{DPI}$, and the density of IBA1+ cells decreased afterwards. At 14 DPI (A), the proportion of the volume occupied by IBA $1^{+}$cells in the lesions (devoid of FluoroMyelin Green stain) was higher in Myd88 $\%$ than WT. Welch's $t$-test. (Error bars: $95 \% \mathrm{Cl} ; n=4-9$ lesions) (C) More myelin lipids accumulated in microglia / macrophages in the lesions of Myd88 mice at 14 $\mathrm{DPI}$, analyzed by the proportion of the volume occupied by both FluoroMyelin Green stain (green) and IBA1 (magenta) (co-localization, white) in the lesions. Welch's $t$-test. (Error bars: $95 \% \mathrm{Cl} ; n=4$ or 5 lesions) (D) The amount of myelin basic protein (MBP) inside IBA $1^{+}$cells was similar in the lesions of $M y d 88^{-\sim}$ and WT mice. 


\section{Astrogliosis was not affected in demyelinated lesions of $M y d 88^{-/}$mice}

At 7 DPI, GFAP-positive astrocytes were found around the demyelinated lesions, which were full of microglia / macrophages. Some astrocytes seemed to be entering the lesions (Figure 3A). At $21 \mathrm{DPI}$, the lesions contained many astrocytes and reduced IBA1-positive cells (Figure 3B). The density of GFAP-positive cells in the lesions of WT and Myd88mice were similar at both 7 and 21 DPI (Figure 3C).

\section{IBA1 GFAP}

(A) 7 DPI

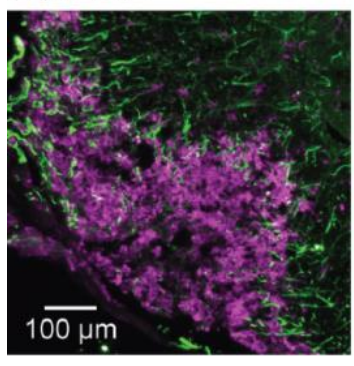

(B) $21 \mathrm{DPI}$

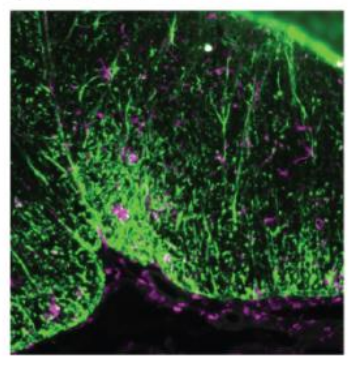

(C)

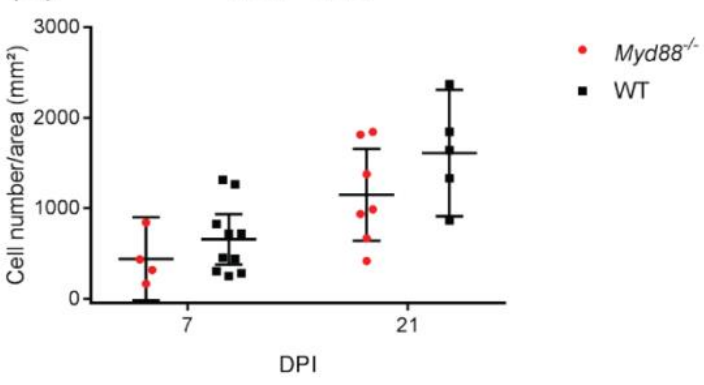

Figure 3. Astrocytes surrounded demyelinated lesions at $7 \mathrm{DPI}$ and were in the lesions at $21 \mathrm{DPI}$. (A) At $7 \mathrm{DPI}$, the lesion core was devoid of $\mathrm{GFAP}^{+}$astrocytes (green) and full of IBA $1^{+}$microglia / macrophages (magenta). (B) At $21 \mathrm{DPI}$, astrocytes reached the lesions, whereas microglia / macrophages left the lesions. (C) The cell density of astrocytes in the lesion core of $M y d 88^{-}$and WT mice was similar at 7 and $21 \mathrm{DPI}$. (Error bars: $95 \% \mathrm{Cl} ; n=4-10$ lesions) 


\section{Myelin debris was taken up faster and in larger amounts than non-myelin membrane by cultured microglia}

Upon demyelination or the damage to other membranous structures in the CNS, microglia respond by internalizing damaged membrane, usually by phagocytosis. To examine the specificity of this response to the type of damage, the phagocytosis of purified myelin debris and non-myelin membrane by cultured microglia was analyzed. Microglia were shaken off WT mixed glial cultures and cultured overnight in DMEM containing 20\% L929 cell-conditioned medium, 10\% FCS, $1 \mathrm{mM}$ sodium pyruvate and penicillin/streptomycin. The cells were washed with DMEM containing $1 \%$ BSA and $1 \mathrm{mM}$ sodium pyruvate (DMEM/BSA medium), and treated with PKH67-labeled myelin or non-myelin fraction at a concentration of 1,10 or $30 \mu \mathrm{g} / \mathrm{mL}$ in DMEM/BSA medium for 1, 2, 4 or $16 \mathrm{~h}$. Microglia phagocytosed myelin (Figure 4A) and non-myelin fraction (Figure 4B) in vitro, but there was more myelin than non-myelin fraction inside the cells at each time point. The amount of labeled membrane in each cell was quantified by the integrated intensity of the fluorescence emitted by PKH67 that was localized within the cell stained by lectin. The violin plot of the intensity in each microglial cell (in log2 scale) showed the diversity in the amount of membrane in each cell (Figure 4E). The mean and $95 \%$ confidence interval (Cl) of the intensity in each cell indicated that more myelin than non-myelin fraction was phagocytosed by cultured microglia with a faster kinetics (Figure 4D).

The western blot of myelin and non-myelin fraction for MBP showed that the non-myelin fraction had little myelin proteins (Figure 4F). The quantification of the integrated intensity of MBP bands suggested that the non-myelin fraction contained around $2.311 \%$ (mean of $0.943 \%$ and $3.678 \%$ ) myelin proteins.

Since myelin has higher lipid content than other membrane, and PKH67 labels lipids, we asked whether the larger apparent amount of myelin in microglia was due to the labeling or the differential lipid content of myelin and non-myelin membrane. The ratio of mean integrated intensity of myelin to non-myelin fraction was higher than the ratio of fluorescence measured from PKH67-labeled myelin to non-myelin fraction (Figure 4G), suggesting that the labeling was not the main factor in the observed difference in the amount of uptake. The ratio of the amount of myelin to non-myelin in cells was also higher than the theoretical ratio of lipid content of myelin to non-myelin membrane $70 \%: 50 \%$. Therefore, cultured microglia indeed phagocytosed more myelin than non-myelin membrane. 
(A) Myelin

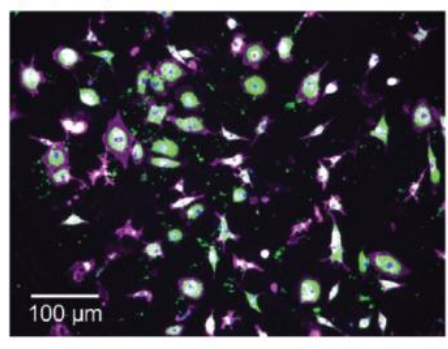

(D)

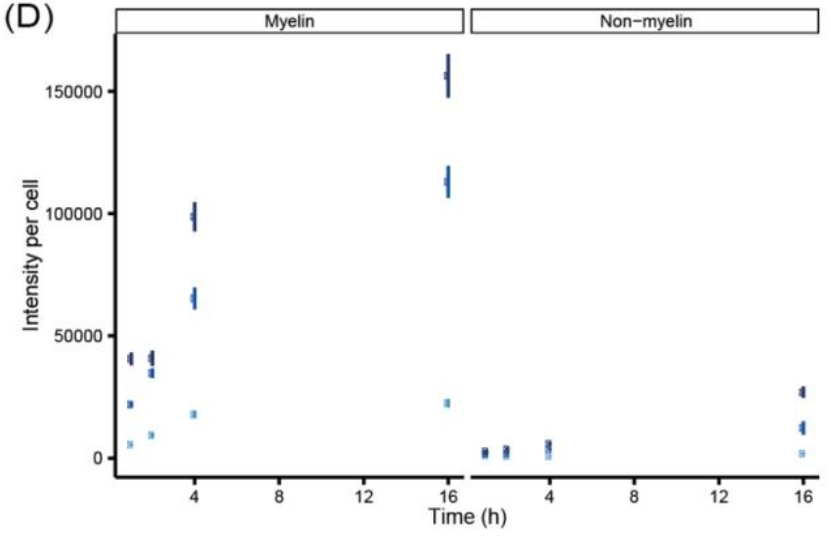

(E)

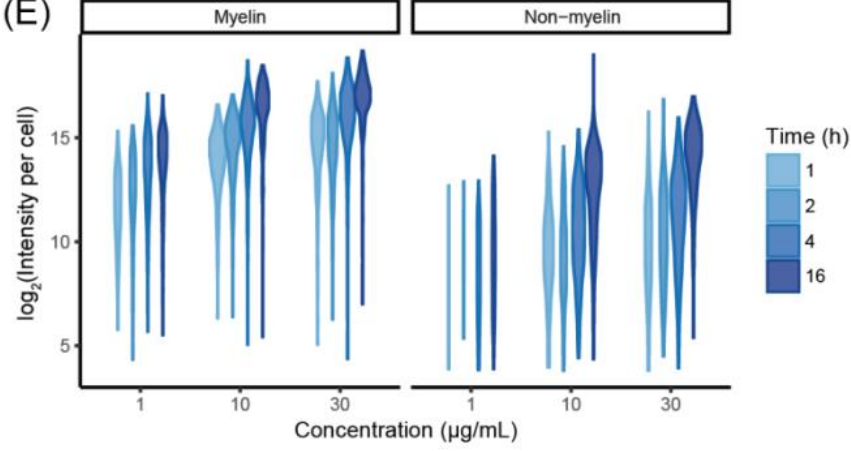

(B) Non-myelin

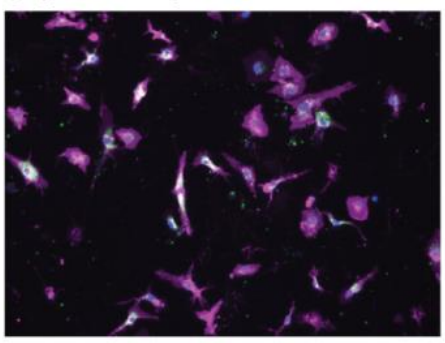

Concentration $(\mu \mathrm{g} / \mathrm{mL})$

$-1$

-10
-30
(C) Control

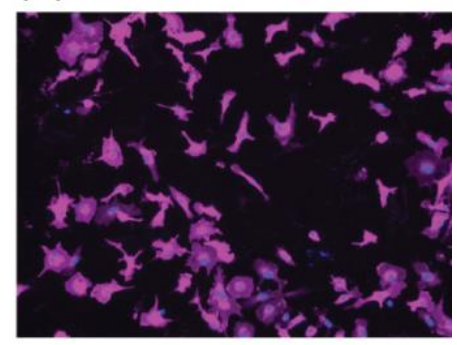

(F) IB: MBP

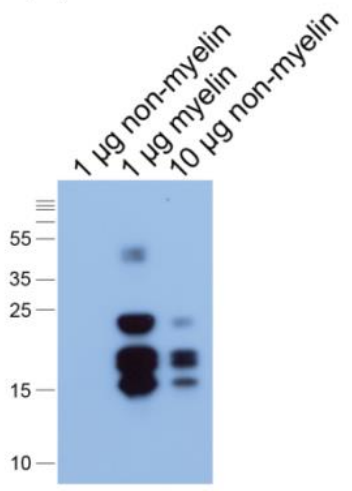

(G) Ratio of myelin to non-myelin membrane

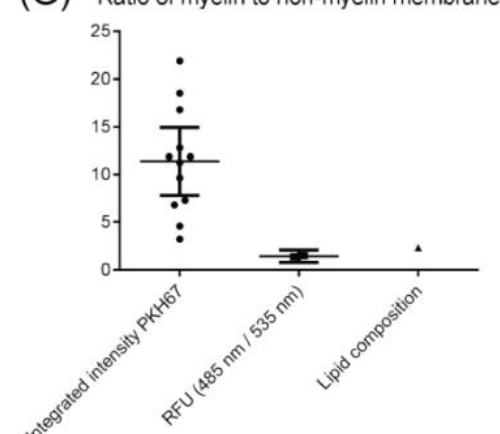

Figure 4. Cultured microglia internalized more myelin debris than non-myelin membrane. WT microglia (magenta) were treated with (A) purified myelin or $(\mathbf{B})$ non-myelin fraction labeled with PKH67 (green) at a protein concentration of 1,10 or $30 \mu \mathrm{g} / \mathrm{mL}$ or (C) PBS control for 1, 2, 4 or $16 \mathrm{~h}$. The amount of myelin in each cell was measured by the integrated intensity of PKH67: (D) mean and $95 \% \mathrm{Cl}$; (E) violin plot of intensity in $\log _{2}$ scale (1 experiment, 2 coverslips per condition, 4 views per coverslip). (F) The purity of non-myelin fraction was checked by western blot for MBP. (G) The higher integrated intensity of PKH67 measured in the cells treated with myelin than in those treated with non-myelin fraction was not due to differential labeling with PKH67. The ratio of mean integrated intensity of myelin to non-myelin fraction per cell (at each concentration and time point) was higher than the ratio of theoretical lipid composition of myelin $(70 \%)$ to other membrane (50\%), which was higher than the ratio of measured fluorescence intensity of PKH67-labeled myelin to non-myelin fraction. (Error bars: $95 \% \mathrm{Cl}$ ) 


\section{The amount of myelin debris in cultured microglia}

WT and $M y d 88^{-/}$microglial cell cultures (MACS) were incubated with serum-free TCC medium overnight, and treated with PKH67-labeled myelin at the same time for the comparison of the amount of myelin uptake in each experiment.

For a short exposure to myelin debris, microglia were treated with $30 \mu \mathrm{g} / \mathrm{mL}$ myelinPKH67, and chased in TCC medium for 15 or 30 (Figure 5A and B). The membrane of the fixed cells was stained with lectin. The images were acquired as z-stacks of 6 optical slices (0.5 $\mu \mathrm{m}$ apart) using the confocal microscope. Maximum intensity projection images of the z-stacks were analyzed using CellProfiler. The amount of myelin in each cell was quantified by measuring the integrated intensity of the thresholded images of the PKH67 channel that was enclosed by the cells. The amount of myelin debris was similar in the cells of different genotypes 15 min after the treatment with myelin debris. The remaining myelin debris attached to the coverslips got phagocytosed by microglia, and therefore more myelin was found in the cells 30 min after treatment than 15 min after treatment. WT microglia phagocytosed more myelin debris on average than $M y d 88^{-/-}$microglia $30 \mathrm{~min}$ after treatment (Figure $5 \mathrm{~A}$ and $\mathrm{B}$ ).

After microglia were treated with $8 \mu \mathrm{g} / \mathrm{mL}$ myelin-PKH67 for $2 \mathrm{~h}$, most WT microglia had phagocytosed myelin. In this scenario, WT microglia contained slightly more myelin debris on average than $M y d 88^{-/}$microglia (Figure $5 \mathrm{C}$ and D).
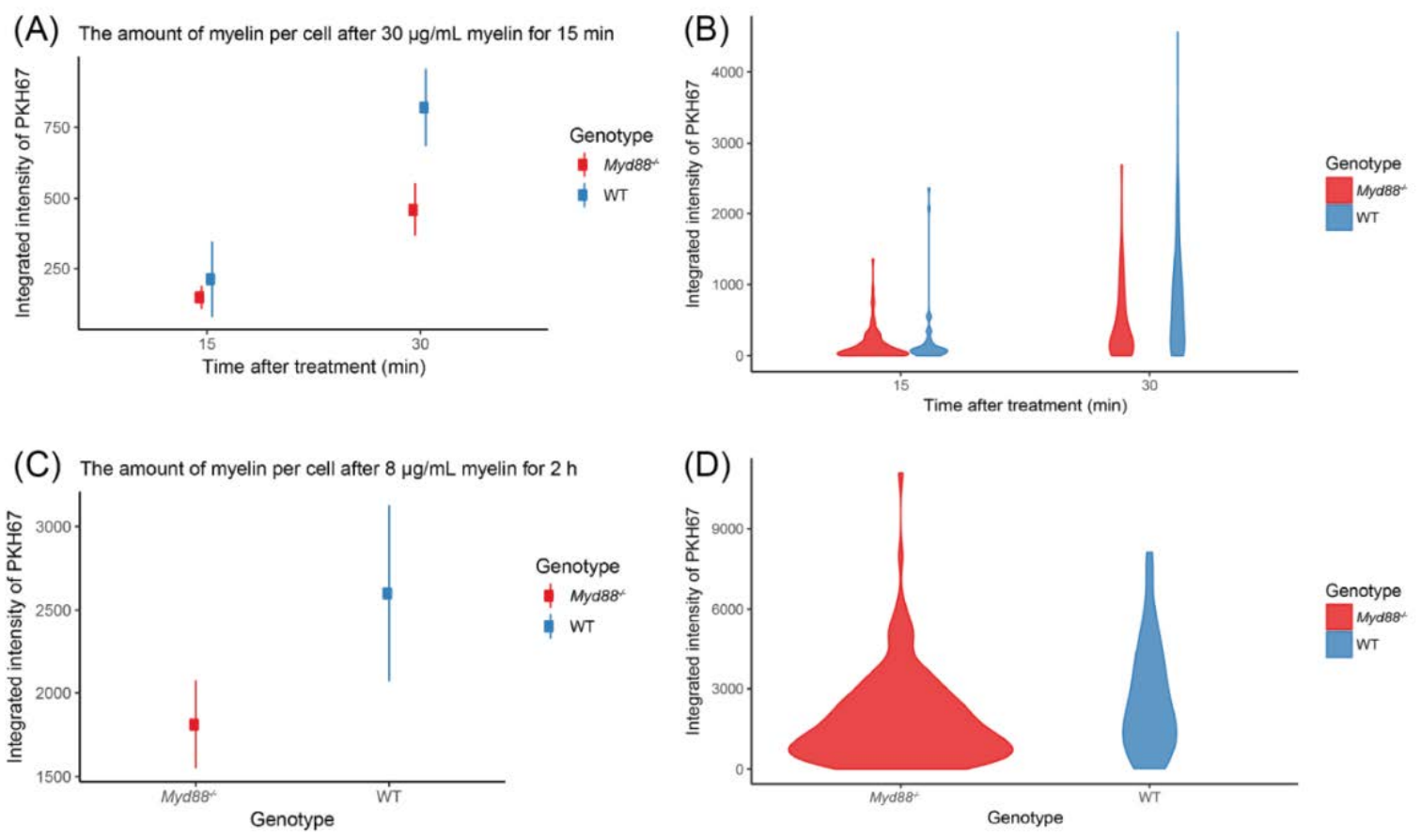
Figure 5. The amount of PKH67-labeled myelin in cultured microglia measured by the integrated intensity of PKH67 in each cell.

In (A) and (B), microglia were treated with $30 \mu \mathrm{g} / \mathrm{mL}$ myelin debris for $15 \mathrm{~min}$, and incubated with medium for 15 or $30 \mathrm{~min}$. In (C) and (D), microglia were treated with $8 \mu \mathrm{g} / \mathrm{mL}$ myelin debris for $2 \mathrm{~h}$. (A) and (C) mean and 95\% Cl; (B) and (D) violin plot (1 experiment each, at least 47 cells analyzed per condition)

\section{Maturation of phagosomes containing myelin debris in cultured microglia}

After overnight incubation with the serum-free TCC medium and the pre-labeling of cell membrane and nuclei, microglia cultures (MACS) were treated with $30 \mu \mathrm{g} / \mathrm{mL}$ PKH67labeled myelin together with LysoTracker for $15 \mathrm{~min}$, and afterwards incubated with TCC medium for $60 \mathrm{~min}$. Both WT and Myd88\% microglia phagocytosed myelin debris. Acidified organelles, endolysosomes or lysosomes, formed in the cells and fused with the phagosomes containing myelin debris (Figure 6A and B). 60 min after myelin treatment, the average number of myelin-positive phagosomes that had fused with LysoTrackerpositive organelles in each cell was higher in WT microglia than in Myd88\% microglia (Figure 6C). The analysis of the area of myelin debris in LysoTracker-labelled organelles also showed that there was less phagosome fusion in $M y d 88^{-/}$microglia than in WT microglia (Figure 6D). 
(A)
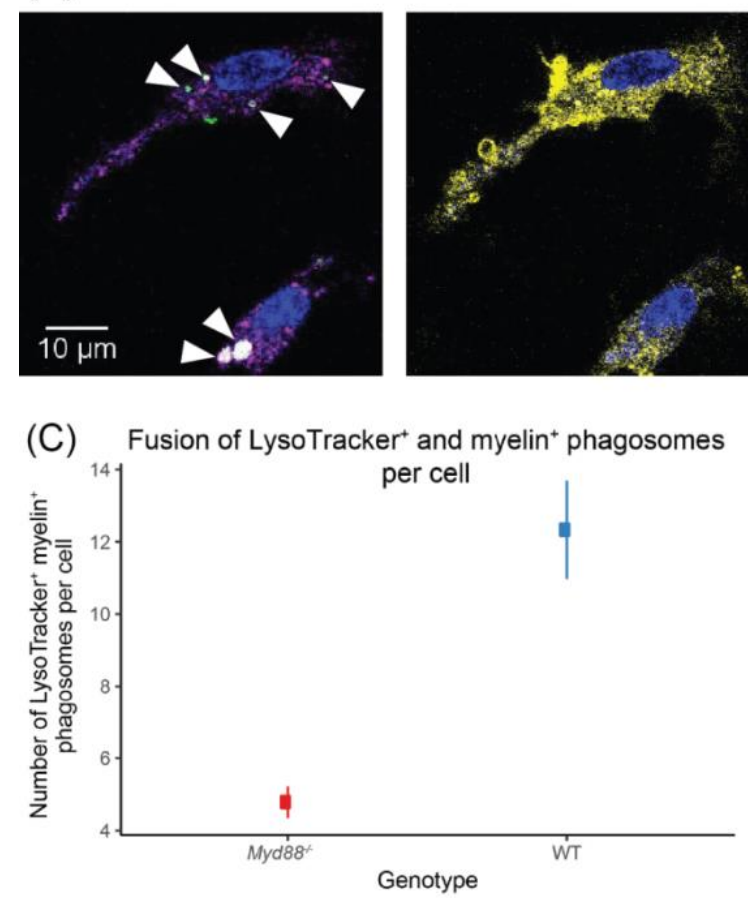

(B)
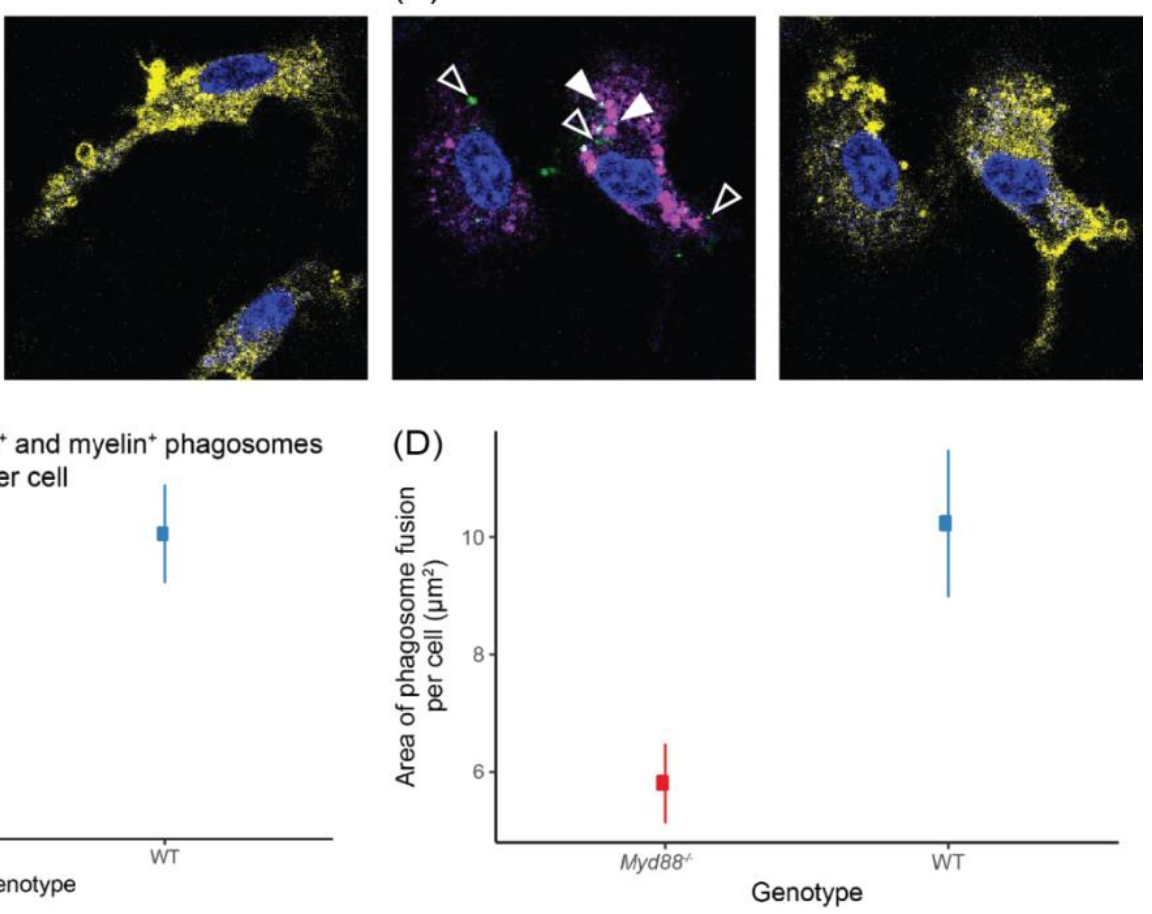

Figure 6. Less fusion of phagosomes containing myelin debris with (endo)lysosomes was observed in cultured $M y d 88^{-/}$than WT microglia 60 min after the treatment with myelin debris.

(A) and (B) In microglia, myelin ${ }^{+}$phagosomes that had fused with LysoTracker (white arrowhead) and myelin ${ }^{+}$phagosomes that had not fused with LysoTracker (open arrowhead) were observed.

(C) The number of myelin ${ }^{+}$phagosomes that had fused with LysoTracker ${ }^{+}$acidified organelles in each cell. (D) The area of the overlap between myelin debris and LysoTracker in each cell. (C) and (D) show the data pooled from $n=3$ independent experiments.

After microglia were treated with $30 \mu \mathrm{g} / \mathrm{mL}$ myelin for $15 \mathrm{~min}$ and incubated with medium for 15min (Figure 7A), the localization of LAMP-2 was altered compared with the control (Figure 7B). The integrated intensity of LAMP-2 immunofluorescence in each cell was high in WT and Myd88 ${ }^{-/}$microglia 15 min after the exposure to myelin debris, and decreased 30 min after treatment. This suggests that the total expression of LAMP-2 protein was increased shortly after the phagocytosis of myelin debris (Figure 7C and D). The mean intensity of LAMP-2 in the perinuclear region decreased more in $M y d 88^{-/}$than WT microglia from 15 to 30 min after myelin treatment (Figure 7E and F). The changes in intensity and localization of LAMP-2 suggest the assembly of LAMP-2 proteins in endolysosomes, which occurred prior to the phagosome maturation observed 60 min after the exposure to myelin (Figure 6). 
(A) LAMP-2 Hoechst

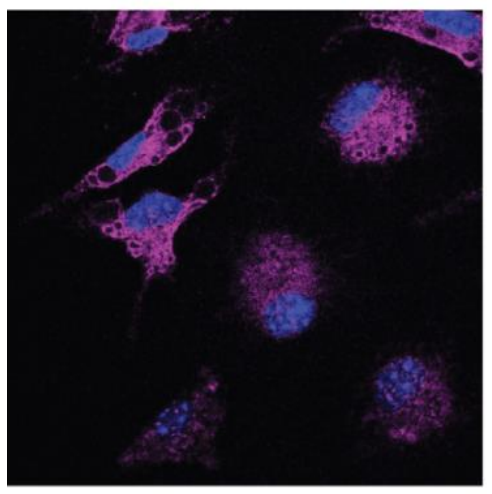

(C)

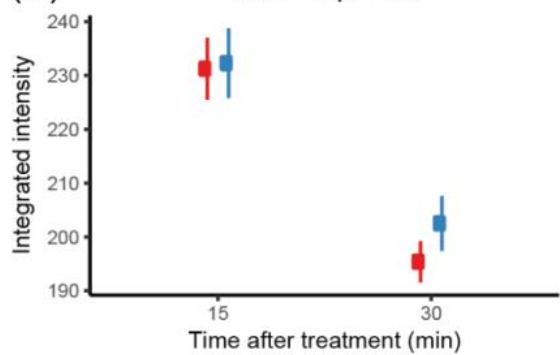

(E)

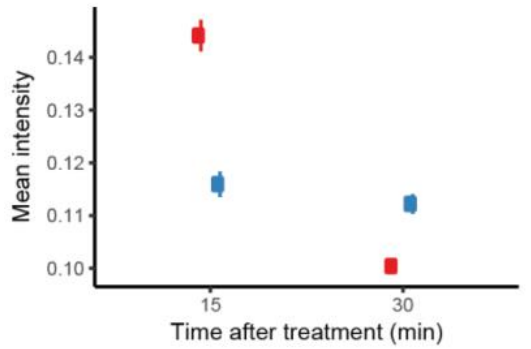

myelin-PKH67 LAMP-2

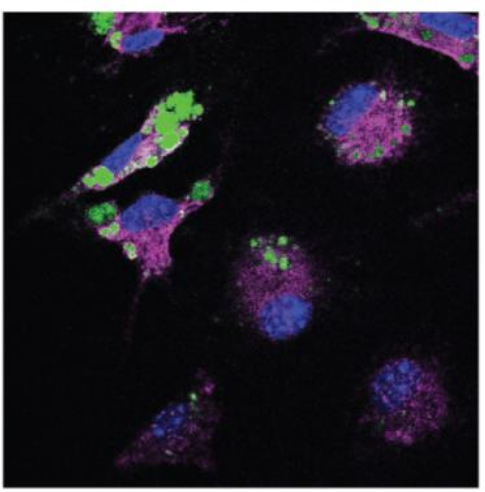

(B)

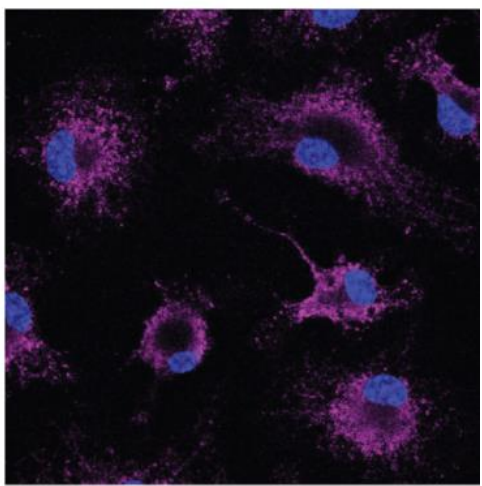

(D)

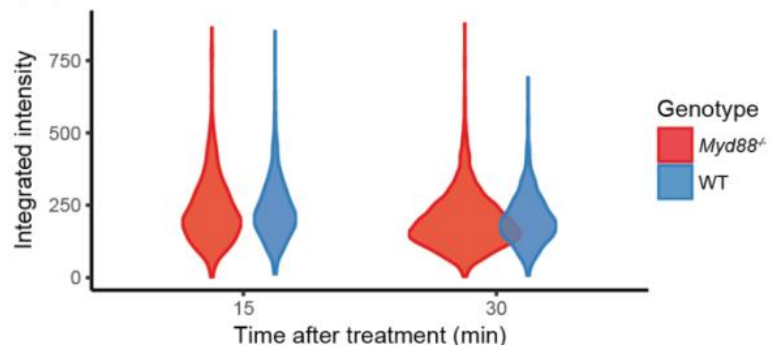

(F)

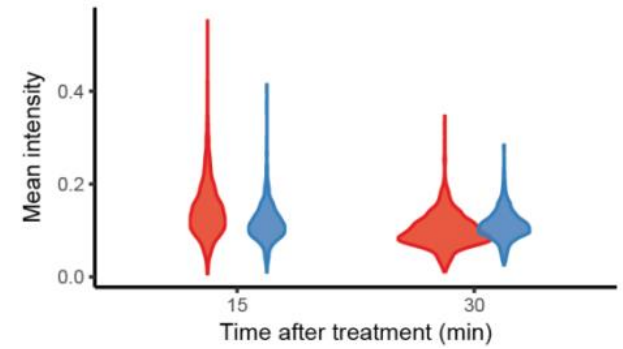

Figure 7. LAMP-2 proteins were assembled in the membrane of endolysosomes containing myelin debris in cultured microglia.

(A) Microglia were treated with $30 \mu \mathrm{g} / \mathrm{mL}$ myelin-PKH67 (green) in TCC medium for $15 \mathrm{~min}$, and incubated with TCC medium for $15 \mathrm{~min}$; (B) control cells (imaged acquired using the $63 \times$ oil objective of a confocal microscope). The localization and fluorescence intensity of ICC for LAMP-2 (magenta) changed after the exposure to myelin debris. (C) and (D) The integrated intensity of LAMP-2 per cell was high 15 min after myelin treatment and decreased 30 min after treatment. (E) and $(\mathbf{F})$ The mean intensity of LAMP-2 in the perinuclear region decreased from $15 \mathrm{~min}$ to $30 \mathrm{~min}$ after myelin treatment in Myd88\% microglia, but the decrease was smaller in WT microglia. (C) and (E) mean and $95 \% \mathrm{Cl}$; (D) and (F) violin plot (The quantification of fluorescence intensity: 1 experiment, 2-4 coverslips per condition, 6 views per coverslip; images acquired using the $20 \times$ objective of a wide-field fluorescence microscope) 


\section{Proteomic analysis of cultured microglia after exposure to myelin debris}

To identify the molecules secreted by microglia after myelin damage, which regulate the responses of OPCs / oligodendrocytes for remyelination, the supernatant of microglia cultures was analyzed by mass spectrometry. WT and Myd88-- microglial cell cultures prepared by MACS were treated with $30 \mu \mathrm{g} / \mathrm{mL}$ myelin debris (or HEPES control) in TCC medium for $4 \mathrm{~h}$, and incubated with DMEM for $16 \mathrm{~h}$. The supernatant was collected for proteomic analysis. Label-free quantification (LFQ) identified around 2000 proteins in each supernatant sample, among which around $10.13 \%$ are annotated as secreted proteins in the Uniprot database. In each comparison of two groups, the relative amount of the proteins identified in the supernatant and the $p$-value were visualized in volcano plots (Figure 8). The amounts of a protein that fulfilled the criteria $\mid \log _{2}$ (LFQ ratio) $\mid>0.5$ and $p$-value $<0.05$ were significantly different in the two groups. 
Supernatant of microglial cell cultures
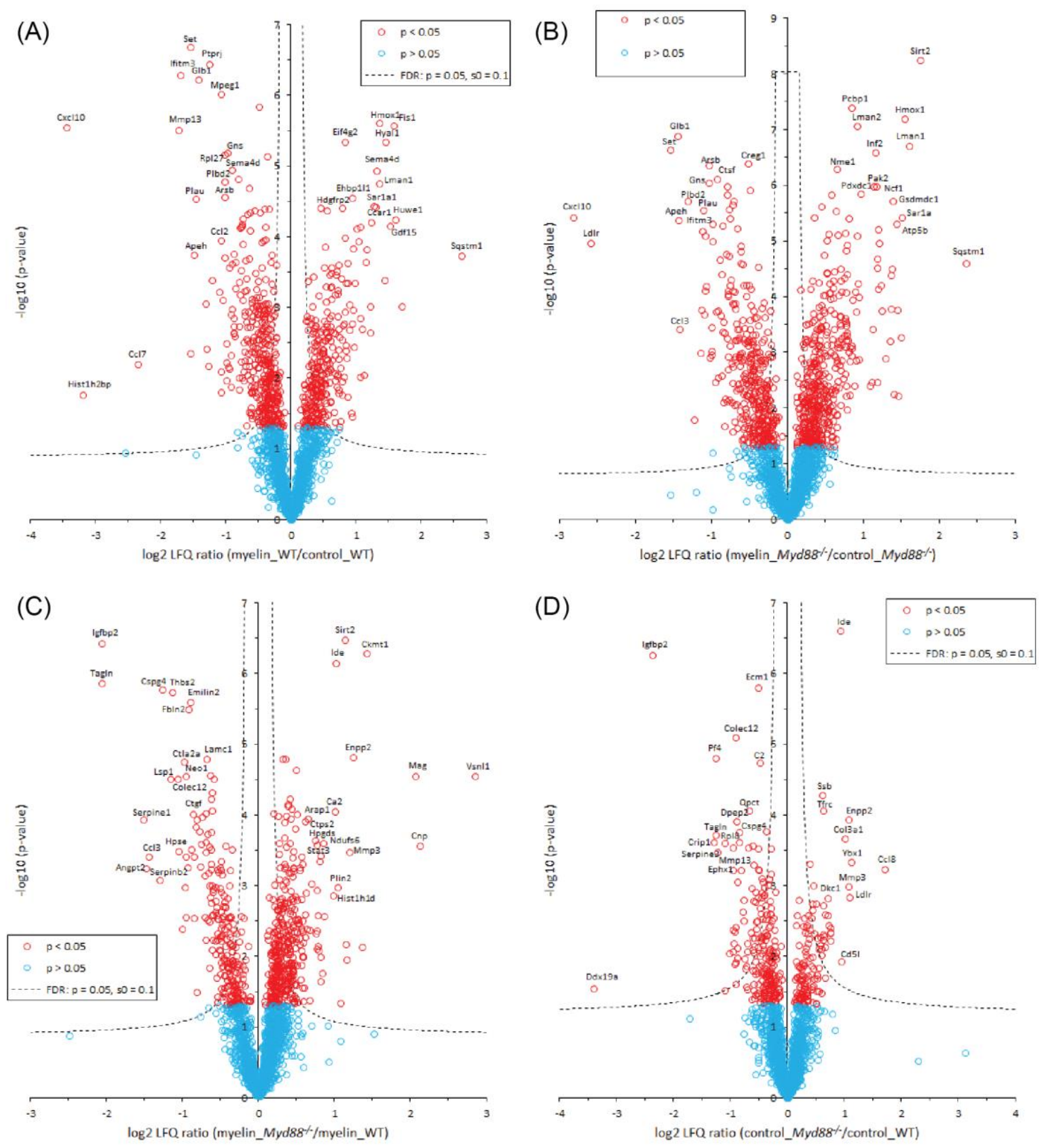

Figure 8. Volcano plots show the relative amount of proteins in the supernatant of microglial cell cultures.

WT and Myd88 microglia were treated with myelin or control for $4 \mathrm{~h}$ in serum-free medium TCC medium, and the supernatant was collected in DMEM for $16 \mathrm{~h}$. (A) WT microglia treated with myelin vs. control. (B) Myd88 $\%$ microglia treated with myelin vs. control. (C) Myd88/ microglia treated with myelin vs. WT microglia treated with myelin. (D) Myd88 microglia control vs. WT microglia control. The proteins with a $p$-value $<0.05$ are shown in red, and $p$-value $>0.05$ are shown in blue. The significant proteins after permutation-based FDR correction are above the dashed curves. ( $n=5$ samples of each condition) 
To select the candidate proteins that were secreted by WT microglia after the exposure to myelin debris but were not secreted by $M y d 88^{-/}$microglia, the differential proteins were selected from the intersection of the two comparisons: myelin_WT / control_WT and myelin_Myd88 ${ }^{-1}$ / myelin_WT (Figure 9). The differentially expressed or secreted proteins identified by comparing WT microglia after myelin treatment and control were likely to function in the response of microglia to myelin damage. Among these genes, the ones that were expressed or secreted at different levels by $M y d 88^{-/}$and WT microglia after myelin treatment might help to explain the altered response of $M y d 88^{-/-}$microglia that led to impaired remyelination in vivo. The proteins identified in both comparisons of the supernatant of myelin_WT / control_WT and myelin_Myd88\% / myelin_WT are listed in Appendix 1. For instance, the abundance of growth/differentiation factor 15 (GDF-15) in the supernatant of WT microglia after myelin treatment was 2.87 fold of control, but its amount in the supernatant of $M y d 88^{-/-}$microglia after myelin treatment was 0.629 fold of that after WT microglia were exposed to myelin debris. GDF-15 is a member of the TGF- $\beta$ superfamily. It is a cytokine and growth factor (Uniprot) that has a neurotrophic role (Machado et al., 2016; Strelau et al., 2000). Another protein, metalloproteinase inhibitor 1 (TIMP-1), was increased in the supernatant of myelin-treated WT microglia (2.70 fold of WT control), but the amount in the supernatant of $M y d 88^{-/}$microglia after the exposure to myelin debris was 0.638 fold of the myelin-treated WT microglia. While TIMP-1 inhibits metalloproteinases, it also functions as a growth factor and regulates integrin signaling (Uniprot). In addition, two proteins involved in the inflammatory response of phagocytosis, collectin-12 (CL-12) and annexin A5, were identified. CL-12 was more abundant in the supernatant of WT microglia after myelin treatment than control, but less abundant in the supernatant of myelin-treated Myd88 ${ }^{-/}$microglia than WT microglia. In contrast, annexin A5 was decreased in the supernatant of WT microglia after the exposure to myelin than control, but more abundant in the supernatant of myelin-treated $M y d 88^{-/-}$microglia than WT microglia. 


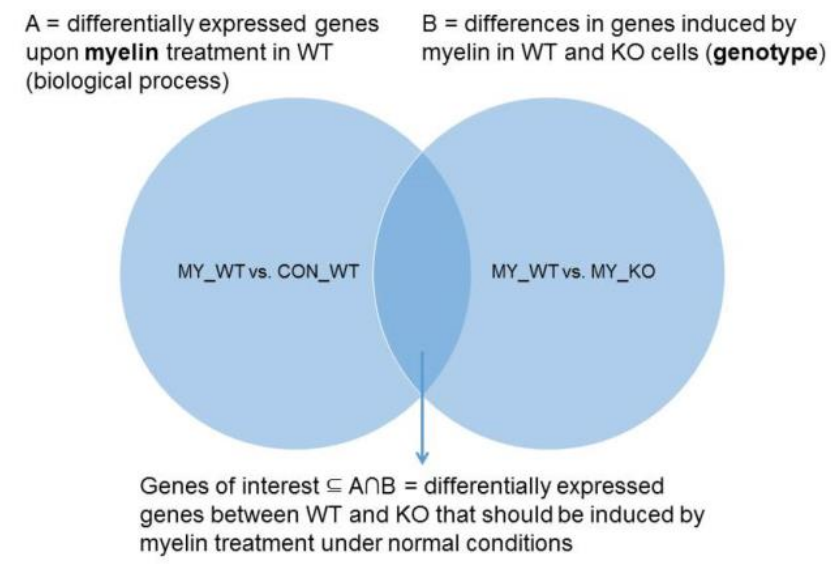

Figure 9. Method to identify the differentially expressed genes from two comparisons.

Set A includes the differentially expressed genes in the comparison myelin_WT/control_WT, which might be relevant to the exposure to myelin debris. Set B includes the differentially expressed genes in the comparison myelin_Myd88 $/$ myelin_WT, which were relevant to the genotype. The intersection of $A$ and $B$ includes the genes that were differentially expressed in cultured $M y d 88^{-1}$ and WT microglia that might be relevant to myelin treatment.

The proteomic data of microglial cell lysate were analyzed in terms of differential gene expression and pathway analysis. In this experiment, WT and Myd88-- microglial cell cultures prepared by MACS were treated with $20 \mu \mathrm{g} / \mathrm{mL}$ myelin debris (or HEPES control) for $4 \mathrm{~h}$ and incubated with DMEM containing $0.2 \%$ BSA for $16 \mathrm{~h}$. Pairwise differential gene expression analyses were visualized as volcano plots (Figure 10). The comparison between control Myd88 ${ }^{-1}$ and WT microglia did not identify significant genes, which suggests that the baseline protein expression profiles of $M y d 88^{-/}$and WT microglia were similar (Figure 10D). After the exposure to myelin debris, a characteristic protein expression profile was induced in WT microglia (Figure 10A). Myelin debris also activated the expression of a set of proteins by $M y d 88^{-/}$microglia (Figure 10B), but the myelininduced protein expression profile of $M y d 88^{-/-}$microglia was distinct from that of WT microglia (Figure 10C). 
Cell lysate of cultured microglia

(A)

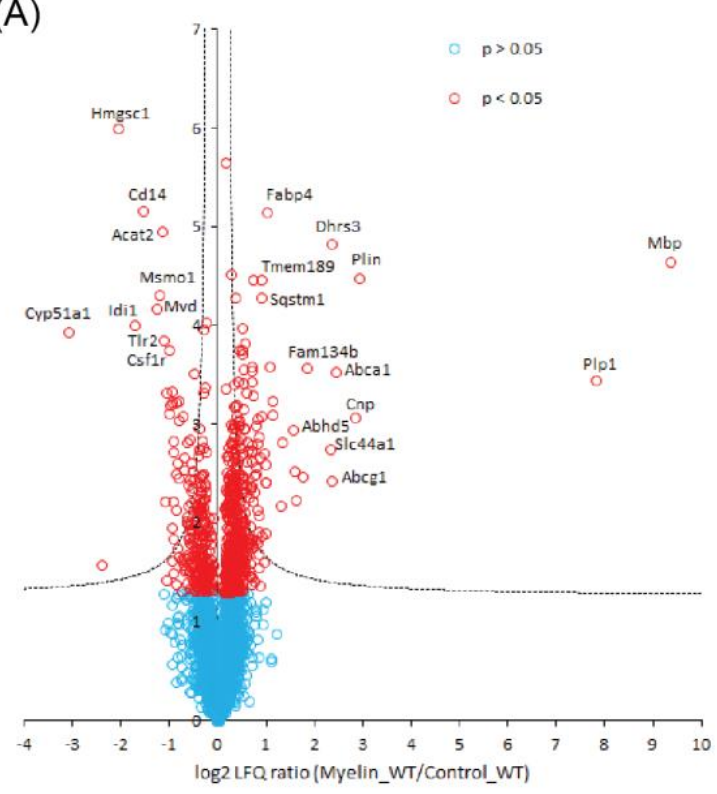

(C)

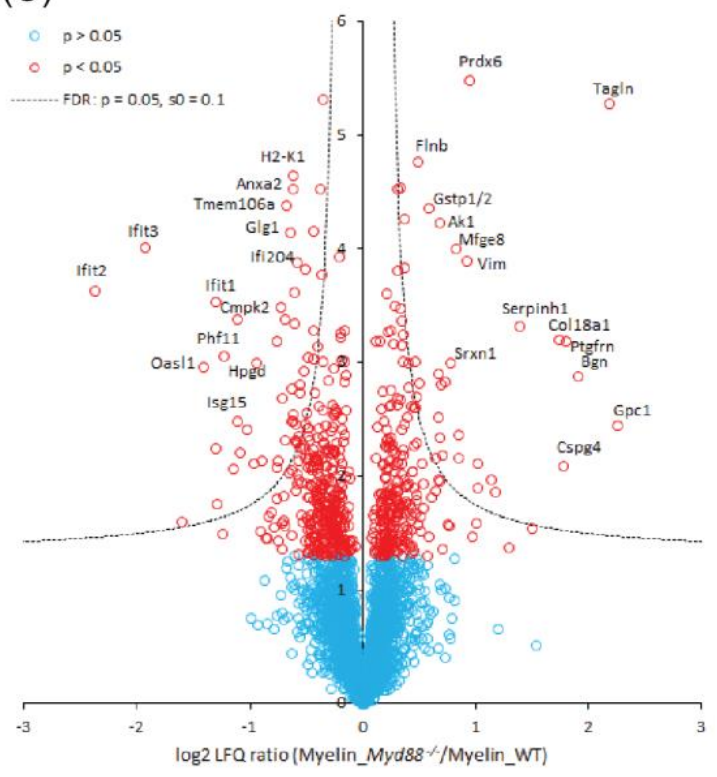

(B)

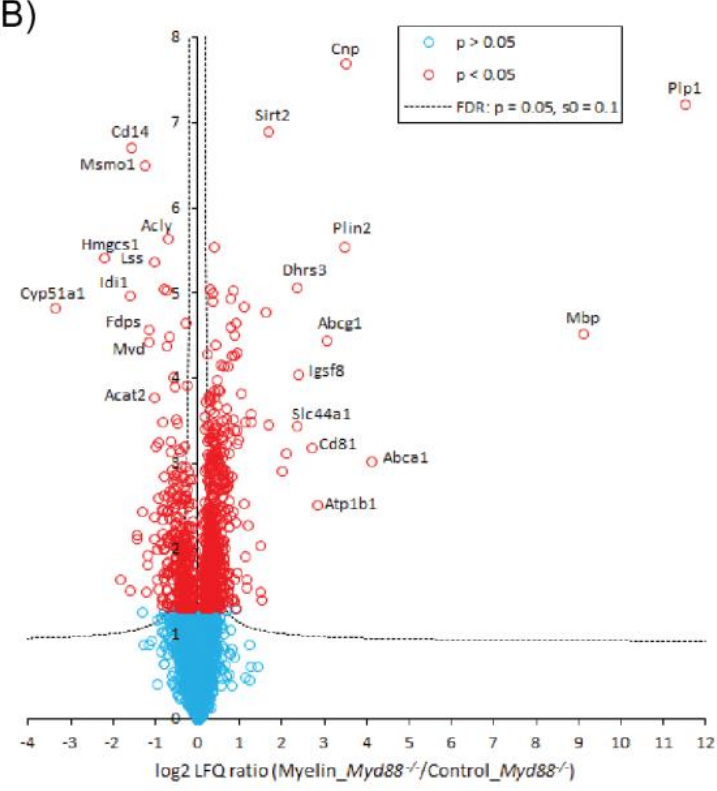

(D)

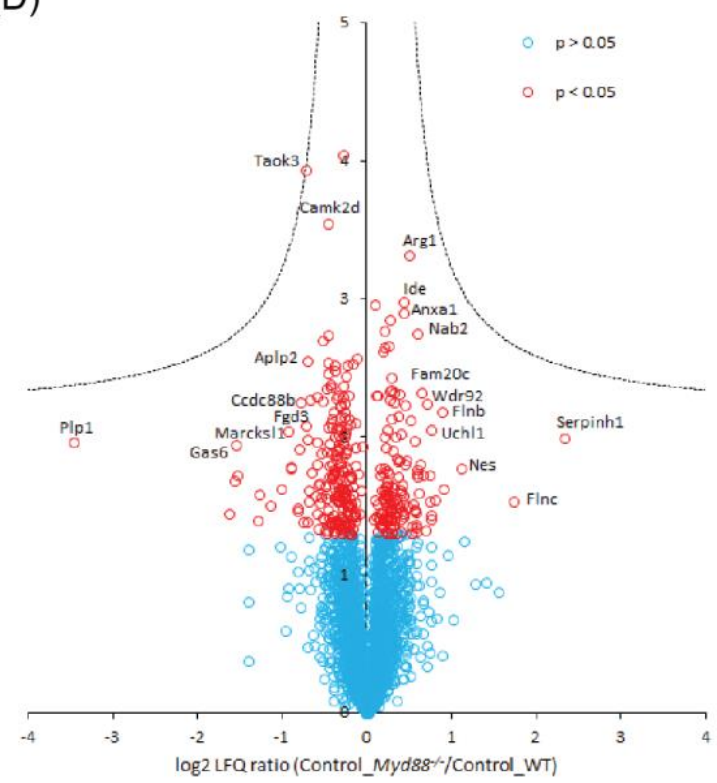

Figure 10. Differential gene expression analyses of the proteomic data of microglial cell lysate. WT and Myd88 microglial cell cultures were treated with myelin or control for $4 \mathrm{~h}$ in serum-free TIC medium and incubated with DMEM containing $0.2 \%$ BSA for $16 \mathrm{~h}$. (A) WT microglia treated with myelin vs. control. (B) Myd88 treated with myelin vs. WT microglia treated with myelin. (D) Myd88\% microglia control vs. WT microglia control. Myd88 - microglia have similar protein expression profile to WT microglia in vitro. The significant genes after FDR correction are above the dashed curves. ( $n=3$ or 4 samples of each condition)

The 10 differentially expressed genes identified in both the comparisons myelin_WT / control_WT and myelin_Myd88\% / myelin_WT (Table 9) were classified into 3 groups: (1) 
the proteins that were decreased in WT microglia upon myelin treatment, but the expression in Myd88/ microglia was higher; (2) the proteins that were increased in WT microglia after the exposure to myelin, but were decreased in $M y d 88^{-/-}$microglia; and (3) the proteins that were decreased in WT microglia after myelin treatment, and were decreased even more in Myd88 ${ }^{-/}$microglia.

Table 9. Differentially expressed genes identified to be significant in the proteomic analysis of microglial cell lysate in both comparisons myelin_Myd88 / myelin_WT and myelin_WT / control WT $\left(\log _{2}\right.$ (expression ratio) $<-0.5$ or $>0.5$, and $p$-value $\left.<0.05\right)$.

(1) Pald1, Camk1d and Tbck were decreased in myelin_WT / control WT but increased in myelin_Myd88\%/ myelin_WT (blue shading).

(2) Pin4, Eif2s3y, Wdr92, Parp12 and Tmem106a were increased in myelin_WT / control WT but decreased in myelin_Myd88-1 myelin_WT (orange shading).

(3) Csf1r and Hpgd were decreased in myelin_WT / control WT and decreased even more in myelin_Myd88\%/ myelin_WT (white shading).

\begin{tabular}{|l|l|l|l|l|l|l|c|}
\hline \multicolumn{2}{|c|}{ Comparison } & \multicolumn{2}{l|}{ Myelin_Myd88-/myelin_WT } & \multicolumn{2}{|c|}{ Myelin_WT/control_WT } \\
\hline Protein names & $\begin{array}{l}\text { Gene } \\
\text { names }\end{array}$ & $\begin{array}{l}\text { log2 (LFQ } \\
\text { ratio) }\end{array}$ & $p$-value & FDR & $\begin{array}{l}\text { log2 (LFQ } \\
\text { ratio) }\end{array}$ & $p$-value & FDR \\
\hline Paladin & Pald1 & 0.84 & $6.74 \mathrm{E}-03$ & + & -0.95 & $2.41 \mathrm{E}-02$ & + \\
\hline $\begin{array}{l}\text { Calcium/calmodulin-dependent } \\
\text { protein kinase type 1D }\end{array}$ & Camk1d & 0.52 & $7.53 \mathrm{E}-03$ & & -0.58 & $3.11 \mathrm{E}-02$ & \\
\hline $\begin{array}{l}\text { TBC domain-containing protein } \\
\text { kinase-like protein }\end{array}$ & Tbck & 0.66 & $1.06 \mathrm{E}-02$ & + & -0.53 & $4.40 \mathrm{E}-03$ & + \\
\hline $\begin{array}{l}\text { Peptidyl-prolyl cis-trans } \\
\text { isomerase NIMA-interacting 4 }\end{array}$ & Pin4 & -0.60 & $4.91 \mathrm{E}-03$ & + & 1.14 & $8.05 \mathrm{E}-04$ & + \\
\hline $\begin{array}{l}\text { Eukaryotic translation initiation } \\
\text { factor 2 subunit 3, Y-linked }\end{array}$ & Eif2s3y & -1.09 & $6.12 \mathrm{E}-03$ & + & 0.95 & $2.50 \mathrm{E}-02$ & + \\
\hline $\begin{array}{l}\text { WD repeat-containing protein } \\
\text { 92 }\end{array}$ & Wdr92 & -0.72 & $4.31 \mathrm{E}-02$ & & 0.63 & $3.40 \mathrm{E}-02$ & + \\
\hline $\begin{array}{l}\text { Poly [ADP-ribose] polymerase } \\
\text { 12 }\end{array}$ & Parp12 & -0.51 & $3.71 \mathrm{E}-02$ & & 0.54 & $2.37 \mathrm{E}-02$ & \\
\hline Transmembrane protein 106A & Tmem106a & -0.68 & $4.12 \mathrm{E}-05$ & + & 0.53 & $2.02 \mathrm{E}-02$ & \\
\hline $\begin{array}{l}\text { Macrophage colony-stimulating } \\
\text { factor 1 receptor }\end{array}$ & Csf1r & -0.86 & $3.43 \mathrm{E}-02$ & & -1.02 & $1.74 \mathrm{E}-04$ & + \\
\hline $\begin{array}{l}\text { 15-hydroxyprostaglandin } \\
\text { dehydrogenase [NAD(+)] }\end{array}$ & Hpgd & -0.95 & $9.84 \mathrm{E}-04$ & + & -0.91 & $6.11 \mathrm{E}-03$ & + \\
\hline
\end{tabular}


The relative expression level of markers for microglia activation and lysosomal function in

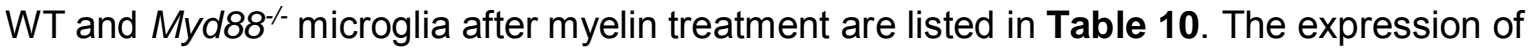
APOE was significantly increased in WT microglia upon the exposure to myelin debris.

Galectin-3 was upregulated to a similar degree in WT and Myd88 ${ }^{-/}$microglia. TREM2 was slightly upregulated by myelin in WT microglia, but the expression was slightly lower in Myd88/- microglia. The expression of CSF1R was decreased upon myelin treatment and

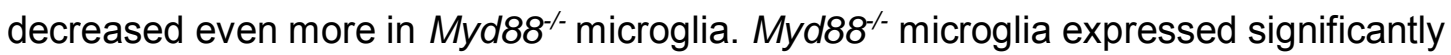
higher level of arginase-1 than WT microglia after myelin treatment, but the induction of arginase-1 by myelin in WT microglia was negligible. LAMP-2 expression level was mildly increased in WT microglia after myelin treatment, but the expression was lower in $\mathrm{Myd}^{-1 /-}$ microglia. The expression of IBA1, CD68 or LAMP-1 was not changed much. The Nos2 gene (encoding iNOS) was not identified by differential gene expression analysis.

Table 10. Selected markers for microglia activation and lysosomal function identified in the proteomic analysis of microglial cell lysate.

A differentially expressed gene in a comparison should meet the criteria $\log _{2}$ (expression ratio) $<-0.5$ or $>0.5$, and $p$-value $<0.05$ (marked red). Significant genes after FDR are marked with "+".

\begin{tabular}{|l|l|c|l|l|l|l|l|}
\hline \multicolumn{2}{|r}{ Comparison } & \multicolumn{2}{c|}{ Myelin_Myd88-/myelin_WT } & \multicolumn{2}{c|}{ Myelin_WT/control_WT } \\
\hline Protein names & $\begin{array}{l}\text { Gene } \\
\text { names }\end{array}$ & $\begin{array}{l}\text { log2 (LFQ } \\
\text { ratio) }\end{array}$ & $p$-value & FDR & $\begin{array}{l}\text { log2 (LFQ } \\
\text { ratio) }\end{array}$ & $p$-value & FDR \\
\hline $\begin{array}{l}\text { Allograft } \\
\text { inflammatory factor } \\
1 \text { (IBA1) }\end{array}$ & Aif1 & -0.56 & $1.21 \mathrm{E}-01$ & & -0.13 & $7.36 \mathrm{E}-01$ & \\
\hline Galectin-3 & Lgals3 & 0.06 & $4.61 \mathrm{E}-01$ & & 0.42 & $1.69 \mathrm{E}-02$ & \\
\hline $\begin{array}{l}\text { Macrophage } \\
\text { colony-stimulating } \\
\text { factor 1 receptor }\end{array}$ & Csf1r & -0.86 & $3.43 \mathrm{E}-02$ & & -1.02 & $1.74 \mathrm{E}-04$ & + \\
\hline Arginase-1 & Arg1 & 0.64 & $6.93 \mathrm{E}-03$ & + & 0.25 & $2.47 \mathrm{E}-01$ & + \\
\hline Apolipoprotein E & Apoe & -0.15 & $1.98 \mathrm{E}-01$ & & 1.13 & $5.84 \mathrm{E}-04$ & + \\
\hline $\begin{array}{l}\text { Triggering receptor } \\
\text { expressed on } \\
\text { myeloid cells 2 }\end{array}$ & Trem2 & -0.21 & $1.40 \mathrm{E}-02$ & & 0.41 & $2.34 \mathrm{E}-03$ & + \\
\hline Macrosialin & Cd68 & -0.08 & $4.02 \mathrm{E}-01$ & & 0.12 & $3.43 \mathrm{E}-01$ & \\
\hline $\begin{array}{l}\text { Lysosome- } \\
\text { associated } \\
\text { membrane } \\
\text { glycoprotein 2 }\end{array}$ & Lamp2 & -0.42 & $1.11 \mathrm{E}-02$ & & 0.38 & $3.00 \mathrm{E}-02$ & \\
\hline
\end{tabular}




\begin{tabular}{|l|l|l|l|l|l|l|l|}
\hline $\begin{array}{l}\text { Lysosome- } \\
\text { associated } \\
\text { membrane } \\
\text { glycoprotein 1 }\end{array}$ & Lamp1 & -0.15 & $4.12 \mathrm{E}-02$ & & 0.13 & $3.42 \mathrm{E}-02$ & \\
\hline
\end{tabular}

To identify the molecules and pathways that are involved in the microglia response to myelin debris, pathway analyses were performed for the differentially expressed genes identified in the comparisons of cell lysate "myelin_WT / control_WT", "myelin_Myd88\% I

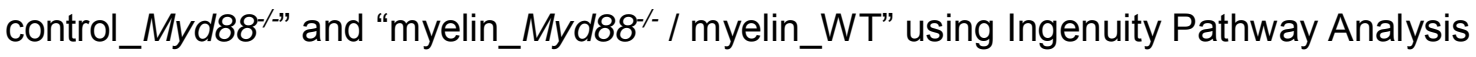
(IPA) and GOrilla. IPA identified comprehensive lists of predicted upstream regulators, mechanistic networks and causal networks. Among the common upstream regulators that were predicted from the comparisons "myelin_WT / control_WT" and "myelin_Myd88 myelin_WT" (Table 11), IFN- $y$-regulated pathways were inhibited $(z$-score $<0)$ in myelin_Myd88 ${ }^{-1}$ vs. myelin_WT but activated ( $z$-score $>0$ ) in myelin_WT vs. control_WT. The target molecules that were predicted to be regulated by IFN- $\gamma$ activation in WT cells upon the exposure to myelin debris are shown in Figure 11A. By comparing Myd88 microglia after treatment with WT microglia after treatment, IFN- $\gamma$ inhibition regulated the target molecules shown in Figure 11B and the predicted mechanistic network shown in Figure 11C.

Table 11. Selected upstream regulators predicted in both comparisons myelin_Myd88-/myelin_WT and myelin_WT/control WT by Ingenuity Pathway Analysis of the proteome of microglial cell lysate. The upstream regulators that were activated in myelin_WT / control WT but inhibited in myelin_Myd88 $1 /$ myelin_WT are labeled with orange shading.

The upstream regulators that were inhibited in myelin_WT / control WT but activated in myelin_Myd88\% / myelin_WT are labeled with blue shading.

The other upstream regulators that were regulated in the same direction in myelin_WT / control WT and in myelin_Myd88\% / myelin_WT are labeled with white shading.

\begin{tabular}{|c|c|c|c|c|c|c|c|}
\hline \multicolumn{2}{|c|}{ Comparison } & \multicolumn{3}{|c|}{ Myelin_WT / control_WT } & \multicolumn{3}{|c|}{ Myelin_Myd88-1/ / myelin_WT } \\
\hline $\begin{array}{l}\text { Upstream } \\
\text { regulator }\end{array}$ & Molecule type & $\begin{array}{l}\text { Predicted } \\
\text { activation } \\
\text { state }\end{array}$ & $\begin{array}{l}\text { Bias- } \\
\text { corrected } \\
\text { z-score }\end{array}$ & $\begin{array}{l}p \text {-value of } \\
\text { overlap }\end{array}$ & $\begin{array}{l}\text { Predicted } \\
\text { activation } \\
\text { state }\end{array}$ & $\begin{array}{l}\text { Bias- } \\
\text { corrected } \\
z \text {-score }\end{array}$ & $\begin{array}{l}p \text {-value of } \\
\text { overlap }\end{array}$ \\
\hline IFNG & Cytokine & - & 0.579 & $1.58 \mathrm{E}-02$ & Inhibited & -2.928 & 3.39E-09 \\
\hline TNF & Cytokine & - & 0.204 & 2.29E-04 & - & -0.48 & $9.32 \mathrm{E}-10$ \\
\hline
\end{tabular}




\begin{tabular}{|c|c|c|c|c|c|c|c|}
\hline IL10 & Cytokine & - & -0.713 & 4.30E-02 & - & 0.739 & $2.08 \mathrm{E}-03$ \\
\hline TGFB1 & Growth factor & - & -0.62 & $3.01 \mathrm{E}-02$ & - & 2.249 & $5.85 \mathrm{E}-04$ \\
\hline AGT & Growth factor & - & -1.68 & 2.00E-02 & - & -0.242 & $1.78 \mathrm{E}-02$ \\
\hline TLR4 & $\begin{array}{l}\text { Transmembrane } \\
\text { receptor }\end{array}$ & - & -1.22 & 3.39E-02 & Inhibited & -1.872 & $1.21 \mathrm{E}-03$ \\
\hline ADRB & Receptor (group) & Activated & 2.992 & $8.55 \mathrm{E}-06$ & - & -0.283 & $1.75 \mathrm{E}-03$ \\
\hline MGEA5 & Enzyme & - & -0.236 & $5.35 \mathrm{E}-04$ & Inhibited & -2.91 & $7.65 \mathrm{E}-04$ \\
\hline IKBKB & Kinase & - & 1.225 & $2.11 \mathrm{E}-02$ & - & -1.418 & $9.49 \mathrm{E}-04$ \\
\hline Mek & Kinase (group) & - & 0.065 & 3.35E-02 & - & 1.638 & $6.29 \mathrm{E}-04$ \\
\hline PPARA & $\begin{array}{l}\text { Ligand-dependent } \\
\text { nuclear receptor }\end{array}$ & Activated & 1.723 & $3.66 \mathrm{E}-06$ & - & -0.576 & $1.37 \mathrm{E}-02$ \\
\hline PPARD & $\begin{array}{l}\text { Ligand-dependent } \\
\text { nuclear receptor }\end{array}$ & - & 0.53 & $1.83 \mathrm{E}-02$ & - & -0.649 & $1.49 \mathrm{E}-02$ \\
\hline PPARGC1A & $\begin{array}{l}\text { Transcription } \\
\text { regulator }\end{array}$ & Activated & 2.054 & 7.76E-03 & - & -0.361 & 3.62E-02 \\
\hline $\begin{array}{l}\text { NFkB } \\
\text { (complex) }\end{array}$ & Complex & - & -0.231 & 3.15E-02 & - & -0.504 & $1.91 \mathrm{E}-03$ \\
\hline JUN & $\begin{array}{l}\text { Transcription } \\
\text { regulator }\end{array}$ & - & -0.172 & $4.88 \mathrm{E}-02$ & - & 0.527 & $1.27 \mathrm{E}-02$ \\
\hline CREB1 & $\begin{array}{l}\text { Transcription } \\
\text { regulator }\end{array}$ & - & 1.552 & 3.21E-07 & - & 0.758 & $1.51 \mathrm{E}-02$ \\
\hline STAT6 & $\begin{array}{l}\text { Transcription } \\
\text { regulator }\end{array}$ & - & 0.742 & $1.72 \mathrm{E}-02$ & - & 1.845 & $1.90 \mathrm{E}-05$ \\
\hline TP53 & $\begin{array}{l}\text { Transcription } \\
\text { regulator }\end{array}$ & Activated & 1.979 & $6.79 E-02$ & - & 2.204 & $1.15 \mathrm{E}-03$ \\
\hline HMGA1 & $\begin{array}{l}\text { Transcription } \\
\text { regulator }\end{array}$ & - & 0.447 & 2.30E-02 & - & 0.468 & 2.76E-03 \\
\hline SIRT1 & $\begin{array}{l}\text { Transcription } \\
\text { regulator }\end{array}$ & - & 2.225 & $7.38 \mathrm{E}-02$ & Activated & 2.697 & 1.62E-04 \\
\hline
\end{tabular}


(A)
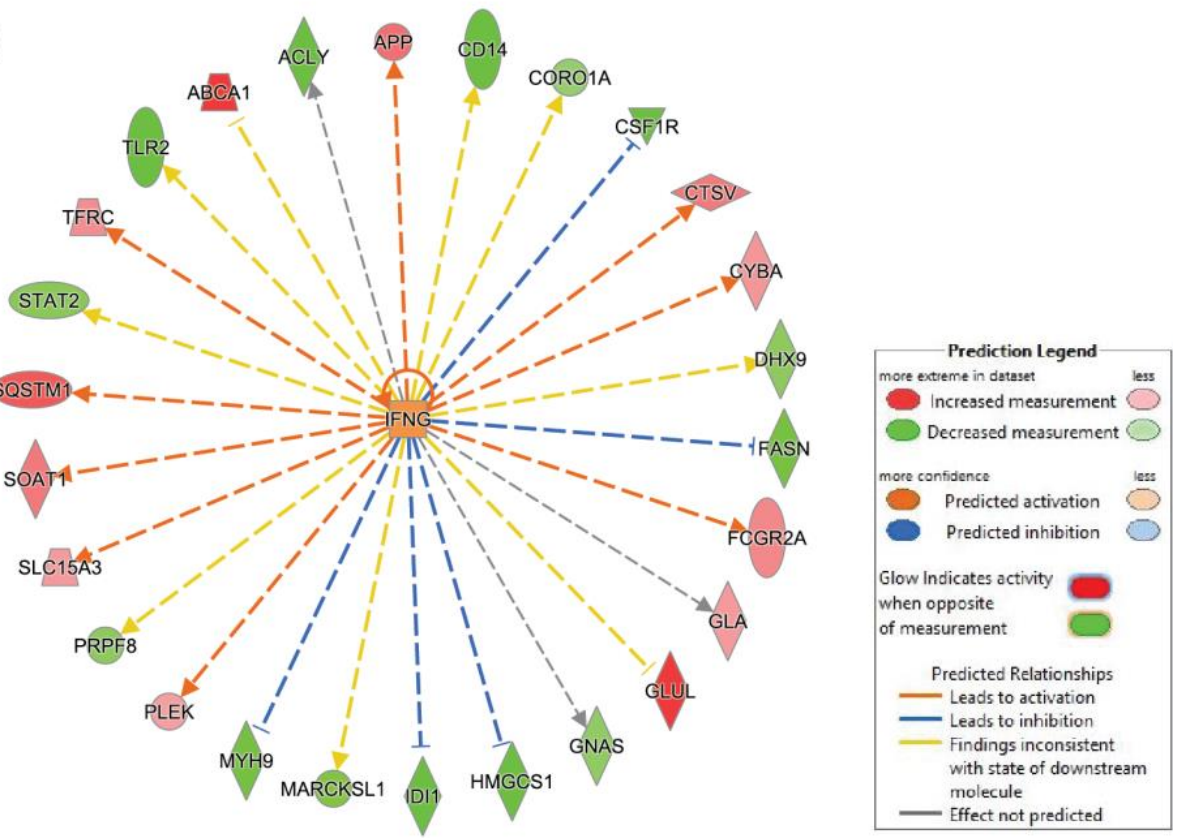

(B)
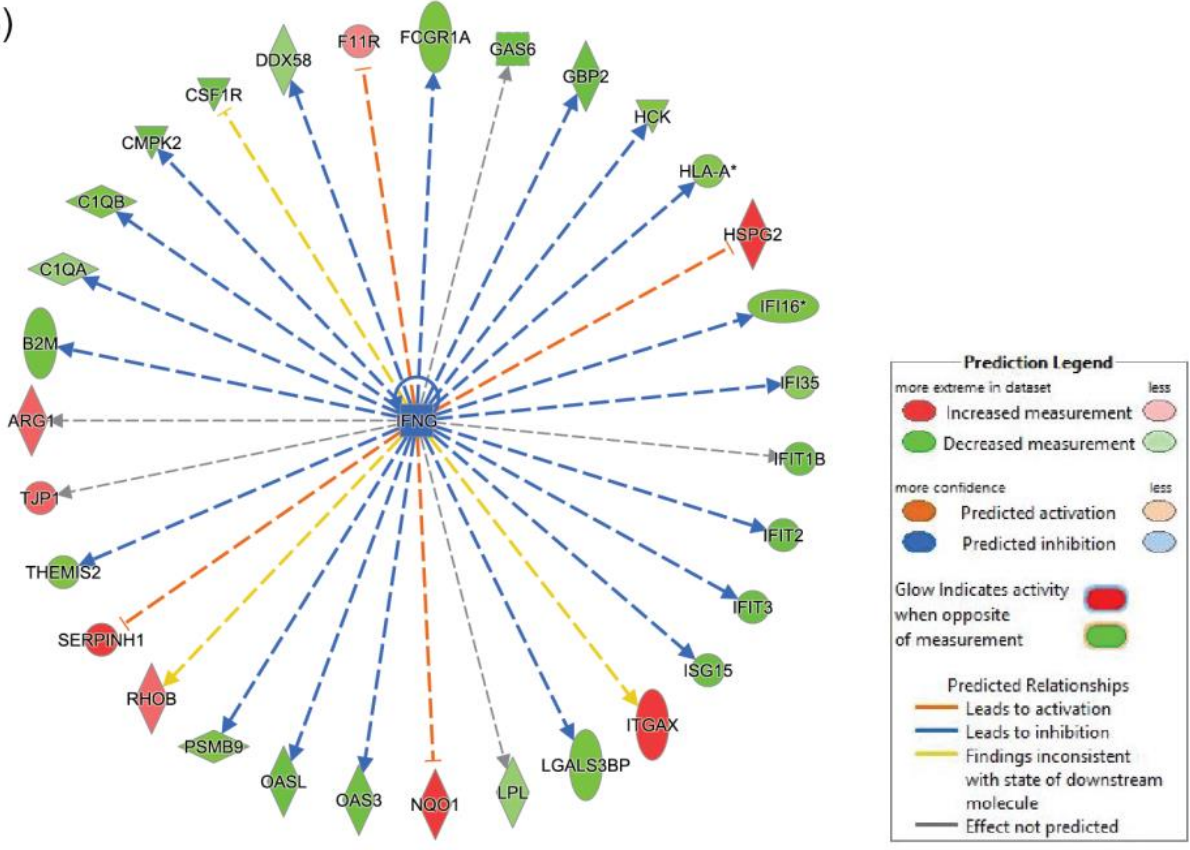

(C)
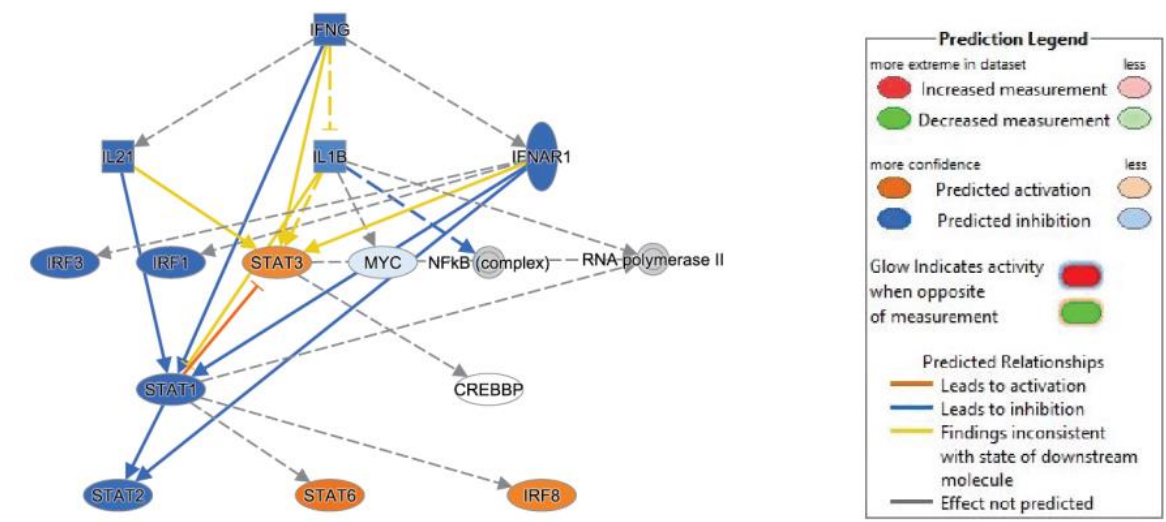
Figure 11. IFN- $\gamma$ was predicted by Ingenuity Pathway Analysis to be an upstream regulator (A) activated in cultured WT microglia after the exposure to myelin debris compared with control; (B) inhibited in cultured Myd88-- microglia compared with WT microglia after the exposure to myelin debris. (C) Mechanistic network regulated by IFN-y predicted in Myd88\% microglia compared with WT microglia after the exposure to myelin debris.

The pathway analysis of the genes that were differentially expressed in cell lysate predicted the pathways with gene enrichment using the GOrilla tool. The exposure to myelin debris induced the expression of genes enriched in lipid metabolic processes, lipoprotein particle organization, and lipid transport in WT microglia (Appendix 2A) $16 \mathrm{~h}$ after a $4 \mathrm{~h}$ of treatment with myelin debris. Myd88-/ microglia, compared with WT microglia, after the exposure to myelin debris expressed genes enriched in immune response, respiratory burst and phagocytosis (Appendix 2B). Myelin debris also led to the enrichment of genes in lipid metabolic processes in $M y d 88^{-/}$microglia but was associated with a broader range of gene enrichment than the response of WT microglia (Appendix $2 A$ and $C)$. 


\section{Transcriptomic analysis of demyelinated lesions}

To compare the gene expression profiles of microglia / macrophages in the demyelinated lesions of $M y d 88^{-/}$and WT mice, the lesions were isolated when the number of microglia / macrophages was the highest (7 DPI). The transcriptomic analysis identified around 210 differentially expressed genes that were statistically significant $\left(\mid \log _{2}\right.$ (ratio) $\mid \geq 2$ and $p$ value $<0.05$ ). Some of these genes encode secreted proteins, including Ccl22 (a chemokine), Inhbb (TGF- $\beta$ superfamily), Lif (an IL-6 family cytokine) and Pkdcc and so on. Other genes are annotated to have functions in signaling, metabolism, lipid processing, inflammation, adhesion, growth and survival, etc.

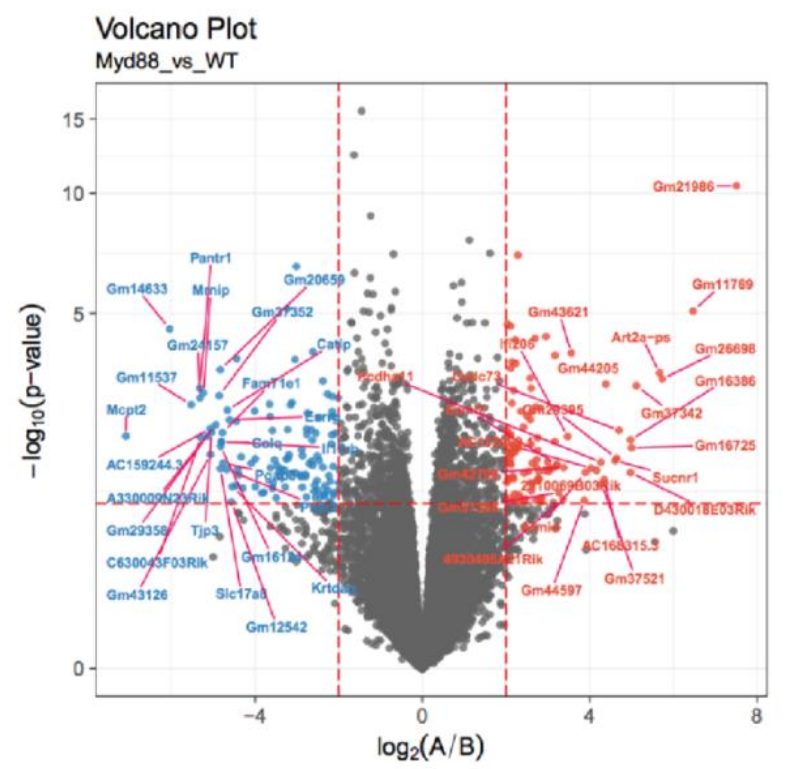

Figure 12. Differential gene expression analysis of the transcriptomes of demyelinated lesions in Myd88\% compared with WT mice.

The lesion samples at 7 DPI were isolated by laser microdissection. ( $n=3 \mathrm{Myd} 88^{-/}$animals; 4 WT animals) 


\section{Assay for myelination in organotypic hippocampal slice culture (OHSC)}

The myelination assay was established in OHSC for testing the effects of molecules on oligodendrocyte differentiation and myelination. The treatments with 3,3',5-triiodo-Lthyronine (T3) for various time and at different concentrations were compared in OHSC and organotypic cerebellar slice culture (OCSC) (Hill et al., 2014). OHSC showed more increase of myelination in response to T3 than OCSC (data not shown), and thus the myelination assay was further optimized in OHSC. The OHSC treated with $80 \mathrm{ng} / \mathrm{mL}$ T3 from 7 DIV for 5 days had increased myelination compared with the control slices (Figure 13). The candidate molecules identified in proteomic and transcriptomic screens can be tested by the myelination assay in OHSC using T3 as a positive control.

\section{MBP NF200}

(A) PBS

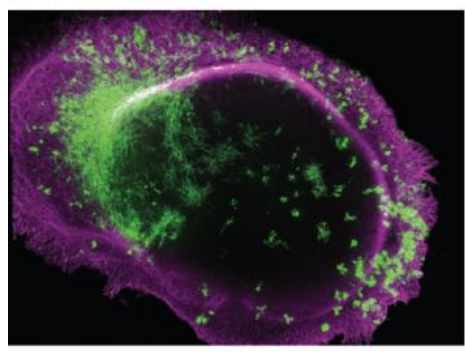

(B) T3

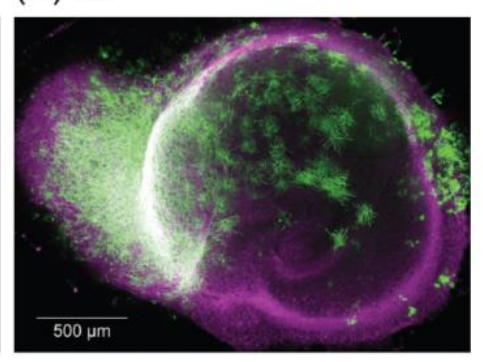

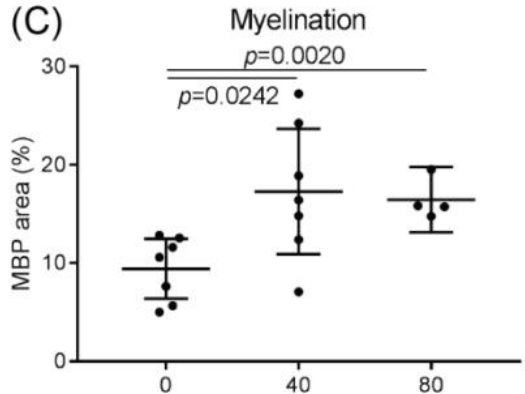

[T3] $(\mathrm{ng} / \mathrm{mL})$

Figure 13. Myelination in organotypic hippocampal slice culture (OHSC).

OHSC treated with $80 \mathrm{ng} / \mathrm{mL}$ T3 (B) had more myelin sheaths (green) compared with control (A). (Neurofilament 200, magenta) (C) Myelination was measured by the proportion of the area of the hippocampal slice that was occupied by $\mathrm{MBP}^{+}$fibers. Unpaired, parametric $t$-test compared with the control (Error bars: $95 \% \mathrm{Cl} ; n=4-7$ slices).

\section{Demyelination model by the injection of lysolecithin in OHSC}

Lysolecithin-induced demyelination model was established in OHSC for studying various cellular events of remyelination and for testing the effects of candidate molecules. The treatment with $0.5 \mathrm{mg} / \mathrm{mL}$ lysolecithin (in the medium and on top of the slices) for $24 \mathrm{~h}$ only induced demyelination on the surface, probably due to insufficient penetration of lysolecithin into the thick slices. The surface of the slices was mainly damaged by cutting during culture preparation, and no clear difference was observed between the surface of the slices treated with lysolecithin and those treated with PBS solvent control. Extended lysolecithin treatment affected the viability of the slice culture (data not shown). Therefore, 
$50 \mathrm{~nL}$ of $0.5 \mathrm{mg} / \mathrm{mL}$ lysolecithin was delivered into hippocampal slices by injection.

Lysolecithin and PBS were labeled with PKH26, which visualized the injection site (Figure 14A). In the slices injected with lysolecithin, the number of microglia increased in the vicinity of the injection site, and many microglia exhibited larger cell bodies and shorter processes. Myelin was disrupted by lysolecithin, forming the debris that was stained by the anti-MBP antibody. Myelin debris in microglia was observed (Figure 14B). In contrast, the injection of PBS left myelin mostly intact and did not alter the ramified morphology of microglia in OHSC (Figure 14C). Therefore, the injection of lysolecithin induced demyelination in OHSC. However, a lot of myelin debris still remained in lysolecithininjected slices 7 days post-injection (Figure 14D) and $14 \mathrm{DPI}$, and many fewer intact myelin sheaths were observed than in control slices (Figure 14E).

(A) IBA1 GFAP PKH26

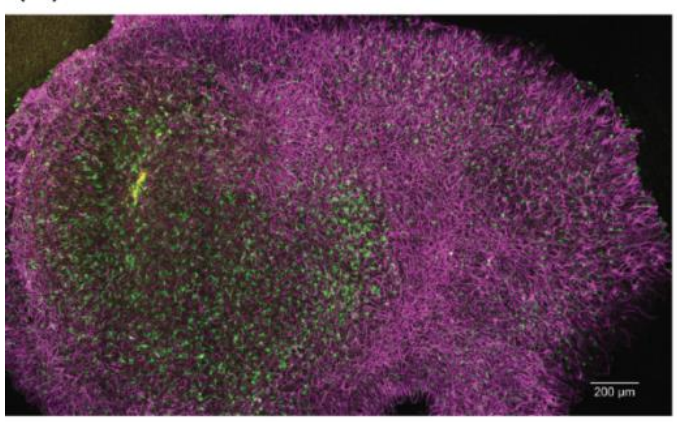

\section{MBP IBA1 PKH26}

(B) Iysolecithin

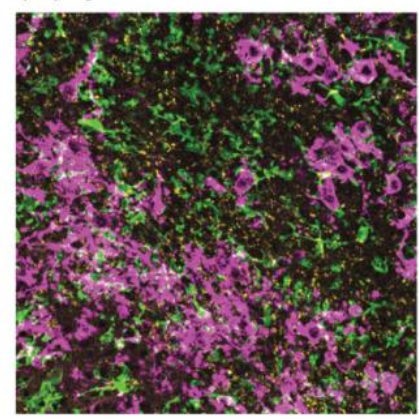

(C) PBS

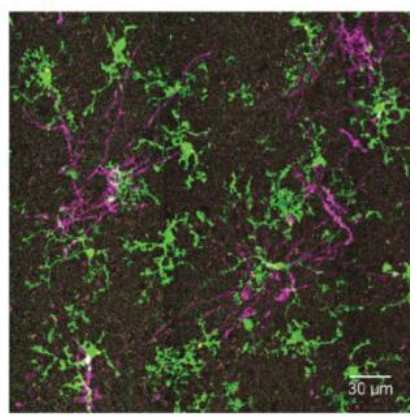

MBP NF-H

(D) lysolecithin

(E) PBS
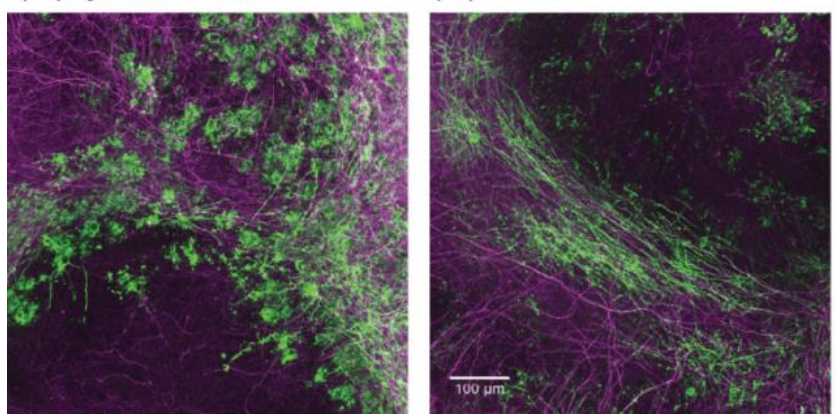

Figure 14. Lysolecithin-induced focal demyelination in OHSC.

After myelination ex vivo (13 DIV), OHSC slices were injected with lysolecithin or PBS labeled with PKH26. (A) PKH26 (yellow) identifies the injection site and the region where lysolecithin or PBS diffused to (2 DPI). At 4 DPI, (B) myelin (MBP, magenta) was disrupted and some myelin debris was inside microglia (IBA1, green) in the slices injected with lysolecithin, whereas (C) myelin was mostly intact in the slices injected with PBS control. After 7 DPI, (D) myelin debris (MBP, green) was still found in lysolecithin-injected slices, and not as many myelin sheaths were present as in (E) PBS-injected slices. 


\section{Autoimmune demyelination model in OHSC}

Antibody and complement-mediated demyelination model was established in OHSC. After myelination for 13 DIV, OHSC was treated with $10 \mu \mathrm{g} / \mathrm{mL}$ anti-MOG Z2 antibody and 5\% guinea pig complement for $72 \mathrm{~h}$. MOG antibody and complement led to the disruption of myelin sheaths (Figure 15A), which did not occur in the slices treated with mouse $\lg _{2 a} \mathrm{~K}$ (isotype control) and complement at the same concentration (Figure 15B). The myelination index of the slices treated with Z2 antibody and complement was significantly decreased by around 51\% compared with the control slices (Figure 15C). MOG antibody and complement induced demyelination in WT OHSC, but remyelination did not occur 7 days post-treatment (DPT) (Figure 15E). Interestingly, most microglia exhibited ramified morphology after $72 \mathrm{~h}$ of treatment with Z2 antibody and complement (Figure 15D). After microglia had been depleted by clodronate, the treatment with Z2 antibody and complement did not cause demyelination in OHSC (Figure 15F). Furthermore, MOG antibody and complement-mediated demyelination was alleviated in Myd88 ${ }^{-1-} \mathrm{OHSC}$ (Figure 15G).

Figure 15. MOG antibody and complement induced demyelination in WT OHSC.

(A) After $72 \mathrm{~h}$ of treatment with $10 \mu \mathrm{g} / \mathrm{mL}$ anti-MOG Z2 antibody and $5 \%$ guinea pig complement, the slices had fewer myelin sheaths (co-localization of MBP (green) and NF-H (magenta)) than (B) the slices treated with the isotype control $\left(\mathrm{mlgG}_{2 \mathrm{aK}}\right)$ and complement. (C) The myelination index $\left(\mathrm{MBP}^{+} \mathrm{NF}-\mathrm{H}^{+} / \mathrm{NF}-\mathrm{H}^{+}(\%)\right)$ was calculated as the voxels occupied by the signals of both MBP and $\mathrm{NF}-\mathrm{H}$ divided by the voxels occupied by the signals of NF-H. Welch's $t$-test (Error bars: $95 \% \mathrm{Cl} ; n=$ 8-10 slices from 2 independent experiments. The data points were normalized to have the same mean myelination index of control slices.) (D) After WT OHSC was treated with Z2 antibody and complement for $72 \mathrm{~h}$, microglia (IBA1, yellow) exhibited ramified morphology. (E) Remyelination did not occur 7 days after the treatment with Z2 antibody and complement (7 DPT). (F) After microglia were depleted from OHSC with clodronate, $\mathrm{Z2}$ antibody and complement did not induce demyelination in WT OHSC lacking microglia. (G) Z2 antibody and complement did not induce significant demyelination in Myd88 OHSC. (Error bars: 95\% Cl. 7 DPT, clodronate treatment and Myd88 OHSC: 1 experiment; $n=4-9$ slices) 
MBP NF-H

(A) $\mathrm{Z} 2$

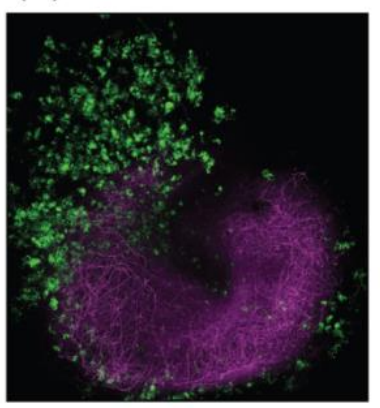

(D) MBP NF-H

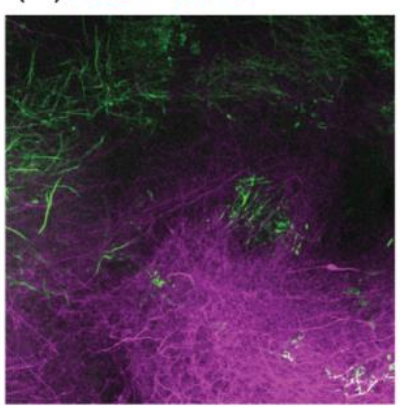

(E)
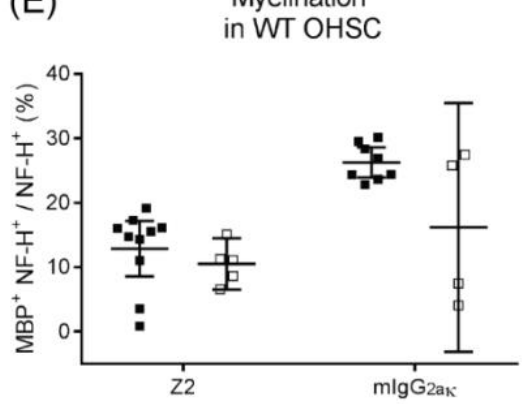

(G)

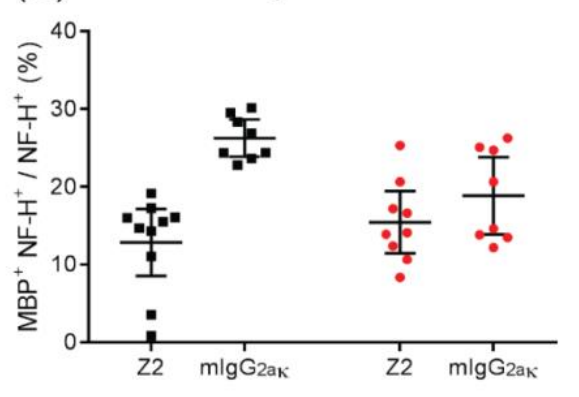

(C) Myelination

in WT OHSC

$p<0.0001$

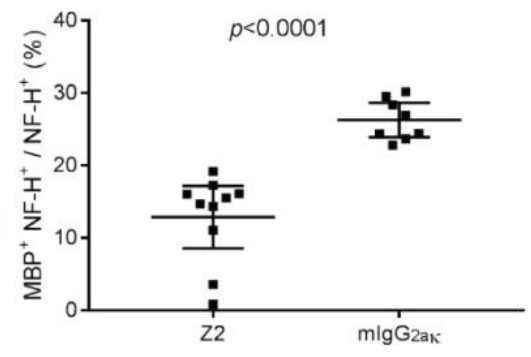

merge

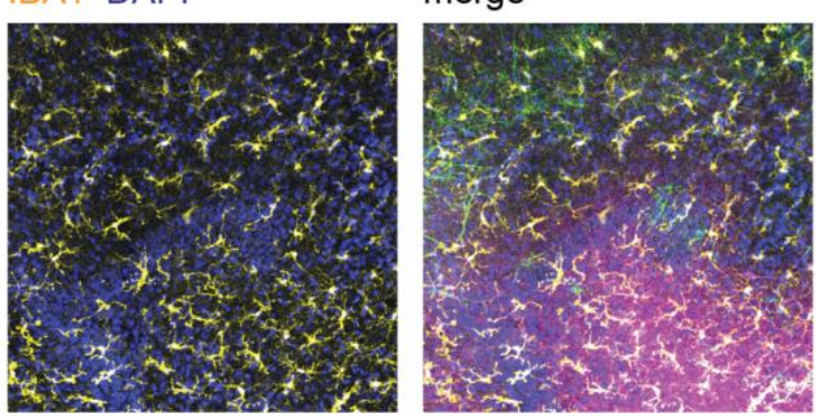

(F)

Myelination

in WT OHSC

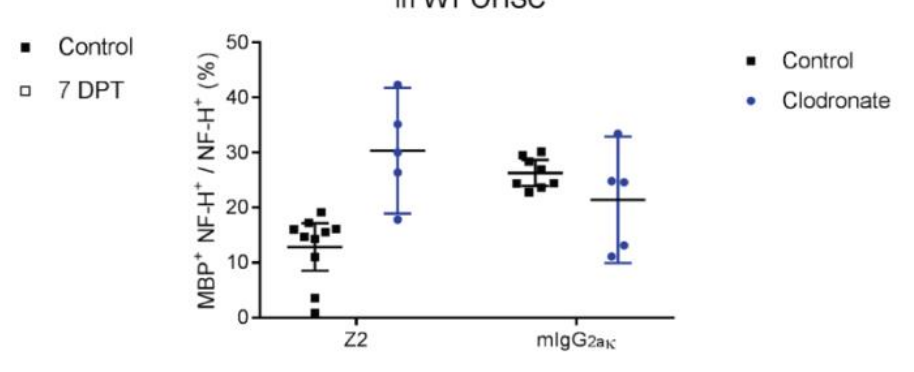

- WT control

- Myd88 


\section{Oligodendrocyte development in Myd88-knockout mice}

Since the increase in the number of OPCs in demyelinated lesions at 7 DPI was abolished

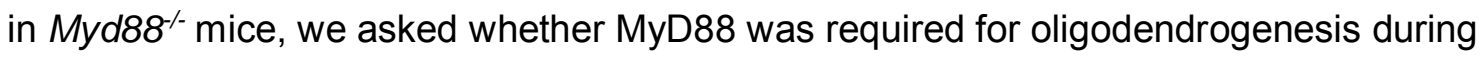
development. The number of OPCs was analyzed in the corpus callosum (CC) of P7 mice, because the density of OPCs is high in the CC of WT pups at this age (Figure 16A). Similar numbers of OPCs (Figure 16B and D) and oligodendrocyte-lineage cells (Figure 16C) were found in the CC of P7 WT and Myd88-- mice, indicating that MyD88 may be dispensable for OPC proliferation during development.

\section{(A) NKX-2.2 OLIG2 DAPI}

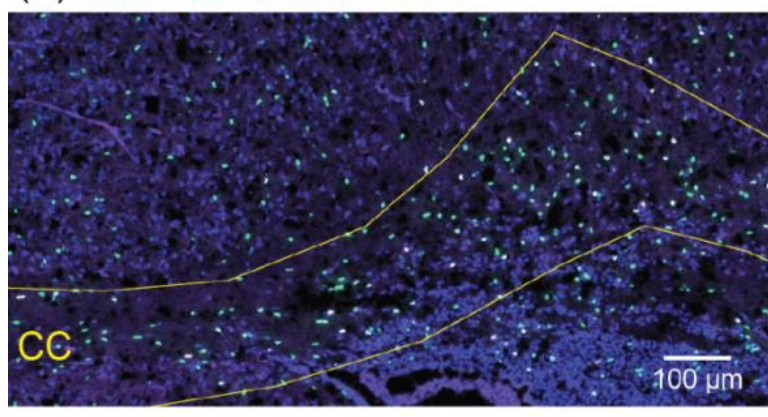

(C)

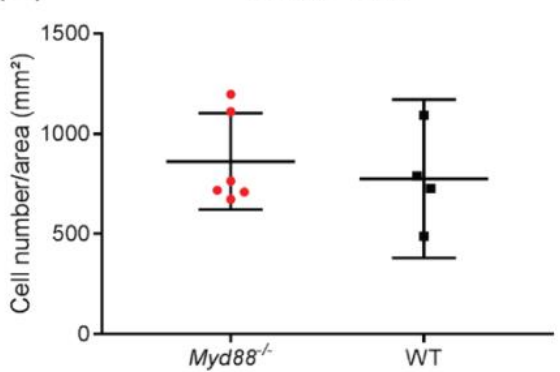

(B)

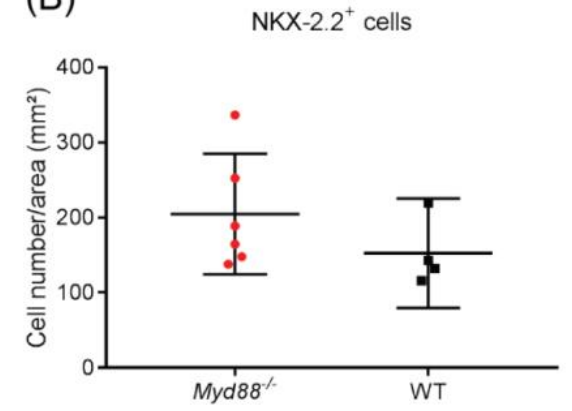

(D) $\quad \mathrm{NKX}-2.2^{+}$cells among $\mathrm{OLIG}^{+}$cells

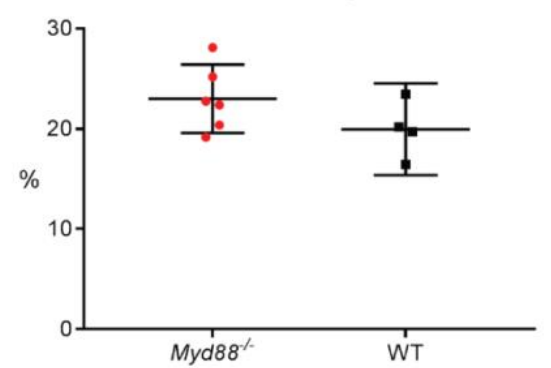

Figure 16. The recruitment of OPCs to developing white matter in the corpus callosum (CC) of Myd88 $/$ mice was not affected.

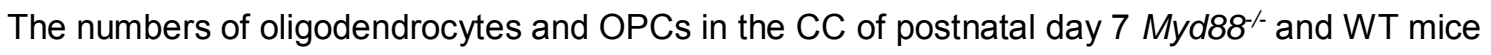
were similar. (A) The nuclei of OPCs in CC were identified by the expression of both the transcription factors NKX-2.2 and OLIG2. (B) The cell density of OPCs in CC was similar. (C) The cell density of oligodendrocyte-lineage cells in CC was similar. (D) The proportion of OPCs among oligodendrocyte-lineage cells was similar. (Error bars: 95\% Cl) 


\section{Discussion}

\section{MyD88-dependent pathways are necessary for the recruitment of OPCs and remyelination}

To understand the roles of microglia - the main resident cells that mediate inflammatory response in the CNS - in remyelination, the lesions of $M y d 88^{-/}$mice were analyzed after focal demyelination induced by the injection of lysolecithin in the spinal cord. Fewer OPCs and early myelinating oligodendrocytes were observed in the lesions of $M y d 88^{-/-}$than wildtype (WT) mice at 7 days post-injection (DPI) (Figure 1E and F). Less recruitment of OPCs may be due to the lack of signals for proliferation and/or migration of OPCs. Consistently, the lesions of $M y d 88^{-/}$mice had fewer mature oligodendrocytes and impaired remyelination at $21 \mathrm{DPI}$ (Figure $1 \mathrm{G}$ and $\mathrm{H}$ ).

The Myd88 gene is predominantly expressed by microglia / macrophages (Brain RNASeq), and therefore they are the main cell types affected by the knockout of Myd88 in the CNS. During the peak of inflammatory response (7 DPI), microglia / macrophages occupy demyelinated lesions at an extremely high density and exhibit amoeboid morphology. As inflammation is gradually resolved, microglia / macrophages leave the lesions (CantutiCastelvetri et al., 2018). Similar density of microglia / macrophages was observed in the core of the lesions of $\mathrm{Myd}_{88^{-/}}$and WT mice at 7 and $21 \mathrm{DPI}$, but more IBA ${ }^{+}$cells remained in the lesions of $M y d 88^{-/}$mice at $14 \mathrm{DPI}$ (Figure $2 \mathrm{~A}$ and $\mathrm{B}$ ). At this timepoint, FluoroMyelin stain detected more myelin lipids accumulated in IBA $1^{+}$cells in the lesions of Myd88/- mice (Figure 2A and C), whereas MBP inside IBA $1^{+}$cells were similar in the lesions of $M y d 88^{-/}$and WT mice at 7, 14 and $21 \mathrm{DPI}$ (Figure 2D). This suggests that microglia / macrophages in $M y d 88^{-/}$mice were defective in degrading myelin lipids after they internalize damaged myelin in the lesions. Remyelination begins from the edge of the lesions, and astrocytes start to repopulate the lesions. Some microglia / macrophages, especially the foam cells containing lipid droplets, remain in the core of the lesions for a long time. The lesion core usually has delayed remyelination and can sometimes have necrosis. Since the density of inflammatory cells can be variable depending on the region of the lesion (unpublished observations by colleagues), microglia / macrophages should be analyzed near the edge as well as in the core of the lesion. Alternatively, all the inflammatory cells could be analyzed in the entire lesion by 3D imaging after tissue clearing. 
The astrocytes in demyelinated lesions appeared similar in WT and Myd88- mice at the time points analyzed (Figure 3). GFAP is expressed by reactive astrocytes in general but cannot distinguish the specific activation phenotypes of astrocytes (Brahmachari et al., 2006; Liddelow et al., 2017). Although astrocytes do not express much Myd88 mRNA (Brain RNA-Seq), they might secrete cytokines and growth factors in response to activated microglia (Hammond et al., 2014; Petkovic et al., 2016), which could be dependent on MyD88-mediated signaling. It was suggested that astrocytes become activated by IL-1 receptor (IL-1R)/MyD88 signaling and secrete pro-inflammatory cytokines and chemokines after spinal cord injury (Pineau et al., 2010). However, it was proposed that MyD88 does not mediate IL-1R signaling in mouse astrocytes, whereas the IL-1R signaling in human astrocytes is dependent on MyD88 or TRIF (Tarassishin et al., 2014). Since no obvious phenotype was found in the astrocytes of $M y d 88^{-/}$mice, the responses of astrocytes during remyelination were not analyzed in this study.

Myd88- mice have reduced inflammatory response and defects in T cell proliferation, but the other aspects of the animals are similar to WT mice (Adachi et al., 1998). Toxininduced demyelination is independent of the actions of T cells (Kremer et al., 2016). Myd88- mice might lack regulatory T cells that promote oligodendrocyte differentiation during remyelination (Dombrowski et al., 2017). However, (1) microglia / macrophages were the predominant cell types in the lesions when the defective OPC recruitment was observed in Myd88\% mice; and (2) whether oligodendrocyte differentiation was normal in the lesions of $M y d 88^{-\sim}$ mice was not conclusive due to fewer OPCs at an earlier time point (Figure 1E-G). Therefore, Myd88\% microglia were further studied to identify the cause of impaired OPC recruitment and remyelination.

\section{MyD88-deficient microglia have impaired phagosome maturation after the phagocytosis of myelin debris}

Microglia maintain the homeostasis by clearing tissue debris and dead cells in the CNS (Hammond et al., 2018), and they are probably the first cells to detect the damage of myelin and phagocytose myelin debris (Franklin and Ffrench-Constant, 2017). To understand the mechanisms of microglial response to demyelination, the phagocytosis of myelin debris, phagosome maturation and the clearance of myelin debris by WT and Myd88\% microglia were compared in cell culture. Microglia isolated by MACS is preferred to the shake of mixed glial cultures, because the morphology of microglia in the MACS 
culture is more diverse and similar to the ramified morphology of homeostatic microglia in vivo, whereas most cells in the culture prepared by shakes have amoeboid morphology of activated microglia. Serum in the growth medium activates microglia and enhances phagocytosis. When the microglia isolated by MACS were cultivated directly in defined, serum-free TIC medium (Bohlen et al., 2017), many cells died within two days (data not shown). Therefore, microglial cell cultures were maintained in the growth medium containing serum and L929 cell-conditioned medium (DMEM/FCS/L929), and the experiments were conducted in serum-free TIC medium after serum-deprivation of the cells. The medium containing TGF- $\beta 2, \mathrm{CSF}-1 / \mathrm{IL}-34$ and cholesterol (TCC/TIC) is by far the minimal medium that keeps the gene expression profile of cultured microglia most similar to those in the brain. The gene expression of microglia is always changed in culture but restored when the cells receive instructive signaling from the intact CNS again (Bohlen et al., 2017). Nevertheless, cell culture models provide the opportunity to study specific cellular mechanisms.

Less fusion of phagosomes containing myelin debris with acidified compartments labeled by LysoTracker was observed in Myd88 ${ }^{-/}$than WT microglia, indicating that phagosome maturation after the phagocytosis of myelin debris is regulated by MyD88-dependent pathways (Figure 6). The microglia cultured in DMEM/FCS/L929 medium had enlarged lysosomes labeled by LysoTracker (data not shown), and therefore the cells were incubated in serum-free TCC medium overnight before experiment for them to reach the baseline condition. Each experiment of WT and Myd88- microglia cultures was performed on the same day for the comparison between genotypes. The fusion of phagosomes containing myelin debris with lysosomes is being validated by electron microscopy (EM).

The phagocytosis of myelin debris by Myd88\% microglia was also analyzed. Less PKH67labeled myelin debris was found in cultured $M y d 88^{-/-}$microglia than WT microglia $30 \mathrm{~min}$ (Figure 5A and $\mathrm{B}$ ) or 60 min after the exposure to myelin (Figure 6E and $\mathrm{F}$ ), and after $2 \mathrm{~h}$ of myelin treatment (Figure $5 \mathrm{C}$ and D). Thus, $M y d 88^{-/}$microglia might phagocytose less myelin debris or the phagocytosis is delayed compared with WT microglia. To test whether the internalization of myelin debris was defective, myelin proteins could be stained in treated microglia using antibodies with or without permeabilization, which can distinguish phagocytosis from the binding of myelin debris (Blander and Medzhitov, 2004).

The regulation of phagocytosis and phagosome maturation is dependent on the phagocytic cargo. The defect in phagosome-lysosome fusion for internalized myelin debris 
is similar to the discovery of impaired phagosome maturation after the phagocytosis of bacteria in $M y d 88^{-/}$macrophages, whereas the maturation of the phagosomes containing apoptotic cells was found independent of MyD88 pathways (Blander and Medzhitov, 2004). To determine whether myelin debris was internalized into the same or separate phagosomes containing bacteria or beads, cultured WT and $M y d 88^{-/}$microglia were treated with myelin debris, heat-inactivated E. coli and latex beads altogether and are being analyzed by EM.

Furthermore, the degradation of internalized myelin debris by microglia is being analyzed. Since phagosome maturation is an essential step for internalized materials to be degraded in the lysosome, we hypothesized that myelin debris would be retained in Myd88/- microglia due to impaired phagosome-lysosome fusion. After WT and Myd88-microglia were treated with myelin debris for $2 \mathrm{~h}$, the amount of myelin in each cell was analyzed at various time points. The amount of myelin debris in microglia / macrophages will be also analyzed in the lesions of WT and $M y d 88^{-/}$mice by immunohistochemistry for LAMP-2, IBA1 and FluoroMyelin at 14 and 21 DPI. FluoroMyelin ${ }^{\mathrm{TM}}$ Fluorescent Myelin Stains (Molecular Probes ${ }^{\mathrm{TM}}$ ) label lipids, especially myelin and lipid droplets inside the cells (Cantuti-Castelvetri et al., 2018; Monsma and Brown, 2012). If persisted expression of LAMP-2 (Mac-3) is observed in microglia / macrophages in the lesions of $M y d 88^{-/-}$mice at a late time point, it might suggest stalled phagosome maturation. However, the amount of FluoroMyelin stain could be the consequence of both phagocytosis and degradation of internalized myelin debris in vivo. If a microglial cell or macrophage containing myelin debris dies, another cell would clear the dead cell and the debris released by the dead cell. After microglia phagocytose damaged myelin, fast phagosome maturation may be required for timely degradation of myelin debris without the chance to present partially digested epitopes from myelin as antigenic peptides (Pauwels et al., 2017). The accumulation of large amounts of cholesterol in phagosomes has been found to impair their fusion with lysosomes in macrophages (Huynh et al., 2008), and the high cholesterol content of myelin might render myelin debris challenging for phagosome maturation to occur in MyD88-deficient microglia / macrophages. Too much accumulation of myelin debris in microglia / macrophages can lead to, for example, the formation of cholesterol crystals and damage to lysosomes, causing maladaptive inflammatory response and impaired remyelination (Cantuti-Castelvetri et al., 2018).

Since microglia phagocytose myelin debris and detect the damage to myelin, we asked whether activated microglia would trigger general tissue repair or specific regenerative 
process in response to demyelination. When microglia were exposed to the same amount of purified myelin debris or non-myelin membrane, they phagocytosed more myelin than non-myelin membrane, and the uptake was faster (Figure 4D and E). The amount of myelin or non-myelin membrane in microglia was quantified from the fluorescence intensity of PKH67 dye, which binds to the lipid regions of cell membrane (Horan et al., 1990; Wallace et al., 2008). The ratio of the integrated fluorescence intensity of myelin to non-myelin membrane in the cells was higher than the ratio of the fluorescence of PKH67labeled myelin to non-myelin fraction (Figure 4G). Therefore, microglia indeed phagocytosed more myelin than non-myelin fraction, and the observation was not due to the artifact of fluorescent labeling. According to the kinetics of the phagocytosis of myelin debris, microglia internalized around half of the maximum amount of myelin after $4 \mathrm{~h}$ of exposure (Figure 4D). After $2 \mathrm{~h}$ of treatment with myelin, the vast majority of microglia had internalized some myelin debris, and therefore this duration of treatment was applied to the clearance assay (Figure 5C). In the assay for phagosome maturation, the uptake of myelin debris happened within 15 min of treatment (Figure 6). The fast and rigorous phagocytosis of myelin debris allows microglia to readily detect the damage to myelin and initiate the responses to demyelination.

\section{Microglia activation and secretion during remyelination}

The activation of microglia was analyzed in both cell culture and demyelinated lesions. The condition after demyelination was modeled in vitro by treating cultured microglia with purified myelin debris. The responses of individual microglia in the same culture was heterogeneous in terms of the amount of myelin debris in each cell (Figure 5B and $D$, Figure 6F, Figure 7, Figure $4 \mathrm{~A}$ and $\mathrm{E}$ ), the expression of activation markers and morphology (Figure S1) and so on. The heterogeneity of microglia activation in vivo (Hanisch and Kettenmann, 2007; Ransohoff, 2016) was recapitulated in cell culture.

The transcriptome and proteome of cultured microglia were analyzed in order to identify the molecules and pathways involved in the activation and secretion of microglia after the exposure to myelin debris. The transcriptome of cultured microglia is being analyzed by next-generation sequencing. Most proteins identified in the supernatant of microglia culture by mass spectrometry are annotated as secreted proteins in the Uniprot database (Figure 8). The proteins that are not annotated as secreted proteins might be shed transmembrane proteins, or secreted unconventionally in serum-free medium; they might 
also come from exosomes or dead cells (communication with Dr. Stephan Müller). The proteins that were found in different amounts in both the comparisons "myelin-treated vs. control WT microglia" and "myelin-treated Myd88\% vs. WT microglia" (Figure 9) are listed in Appendix 1. Some of these proteins will be briefly discussed here.

The TGF- $\beta$ superfamily member growth/differentiation factor 15 (GDF-15) was increased in the supernatant after WT microglia were exposed to myelin debris but less upregulated

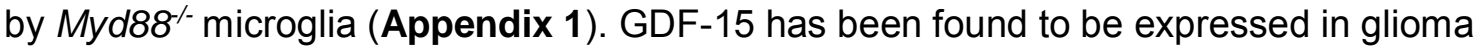
and to enhance the proliferation of glioma cells (Roth et al., 2010). As GDF-15 has cytokine and growth factor activities (Machado et al., 2016; Strelau et al., 2000), it might promote the proliferation of OPCs. The Gdf15 gene is primarily expressed in microglia / macrophages (782.8742 \pm 241.7605 FPKM) in the mouse brain, according to the Brain RNA-Seq database (Zhang et al., 2014). However, the causal relationship between GDF15 and glioma has to be investigated carefully before considering GDF-15 as a candidate molecule for OPC proliferation during remyelination.

The metalloproteinase inhibitor 1 (TIMP-1) was also induced by myelin treatment of WT microglia and less upregulated by Myd88/- microglia (Appendix 1). Apart from inhibiting metalloproteinases, TIMP-1 also has growth factor activity and can regulate integrin signaling (Uniprot). TIMP-1 has been shown to promote OPC proliferation and differentiation and astrocyte proliferation (Moore et al., 2011). TIMP-1\% mice after experimental autoimmune encephalomyelitis (EAE) had increased infiltration of lymphocytes, prolonged activation of microglia / macrophages and more severe myelin damage (Crocker et al., 2006). The expression of Timp1 is low in endothelial cells (11.399 \pm 0.0394 FPKM) and almost undetectable in other mouse brain cell types (Brain RNASeq), but the expression is upregulated during postnatal development (Ulrich et al., 2005) and after injury. TIMP-1 expression was observed in activated astrocytes around the lesions in models of CNS inflammatory disorders by in situ hybridization (Pagenstecher et al., 1998), whereas in another study of EAE, macrophages were identified to be a major source of TIMP-1 by FACS and real-time RT-PCR (Toft-Hansen et al., 2004). According to the secretome analysis, TIMP-1 might be expressed by microglia in response to myelin debris to promote the proliferation of OPCs and astrocytes.

Two proteins involved in phagocytosis-induced inflammation were identified in the secretome of cultured microglia. Collectin-12 (CL-12) was found to be increased in the supernatant of WT microglia after the exposure to myelin debris, but the amount was 
lower in the supernatant of $M y d 88^{-/}$microglia after myelin treatment. Oppositely, less annexin A5 (or annexin V) was secreted by WT microglia upon myelin treatment, but more annexin A5 accumulated in the supernatant of $M y d 88^{-/}$microglia after the exposure to myelin debris (Appendix 1). Phagocytosis of necrotic or lysed cells usually activates inflammation and immune response, contributed in part by members of the collectin family (Henson et al., 2001). Soluble CL-12 has been shown to recognize a fungal pathogen and activate the alternative complement pathway (Ma et al., 2015). On the other hand, phagocytosis of apoptotic cells does not trigger inflammation or immune response. It has been suggested that upon the binding of phosphatidylserine (PS) exposed on the outer membrane leaflet of apoptotic cells to PS receptor (PSR), PSR signaling generates an anti-inflammatory effect. Annexin A5 could block PSR signaling by binding to PS and disrupting the interaction between PS and PSR, thereby allowing the generation of proinflammatory molecules (Stach et al., 2000). If the PS exposed on myelin debris were recognized by surface receptors of microglia, decreased annexin A5 would allow more binding and possibly the expression of anti-inflammatory cytokines by WT microglia later.

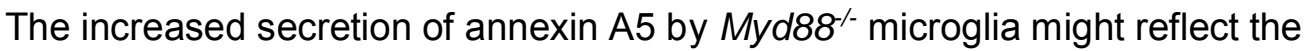
misregulation of the microglial response to myelin debris. The possibility that CL-12 binds to myelin debris and activates complement pathway in microglia or other phagocytes might be also interesting to test.

The proteomic data of the cell lysate of cultured microglia were analyzed in terms of differential gene expression (Figure 10) and pathway analysis. Apolipoprotein E (APOE) was induced in WT microglia after the exposure to myelin debris, which suggests the activation of lipid transport by microglia in vitro. The upregulation of APOE was also observed in the supernatant of cultured microglia, which did not seem to be affected by the lack of MyD88 (data not shown). In WT microglia culture $16 \mathrm{~h}$ after $4 \mathrm{~h}$ of myelin treatment, the so-called "M1" markers were slightly induced, whereas Myd88- microglia showed higher expression of "M2" markers such as arginase-1 and slightly lower expression of "M1" markers (Table 10). Differential gene expression analysis identified 10 genes that were significant when comparing both myelin-treated vs. control WT microglia and myelin-treated Myd88 ${ }^{-/}$vs. WT microglia (Table 9). Further analysis of the proteome of microglial cell lysate was conducted using Ingenuity Pathway Analysis (IPA).

Among the upstream regulators predicted in both comparisons (Figure 9), IFN- $\mathrm{Y}$ regulated pathways were activated in WT microglia after the exposure to myelin debris, but were inhibited in Myd88\% microglia after myelin treatment compared with WT microglia 
(Table 11). In WT microglia, myelin debris induced the expression of some proteins that function in cholesterol metabolic process or cholesterol transport. The upregulation of the sterol O-acyltransferase 1 (Soat1) and the ATP-binding cassette sub-family A member 1 (Abca1) was predicted to be activated by IFN-Y-regulated pathway(s) directly or indirectly. The increase of cathepsin L1 (encoded by the Cts/gene; the human ortholog cathepsin L2 is encoded by the CTSV gene), a protease that is important for protein degradation in lysosomes, suggests the activation of lysosomal function in WT microglia after the exposure to myelin debris (Figure 11A). By comparing the proteome of $M y d 88^{-/-}$and WT microglia after myelin treatment, IPA predicted a mechanistic network regulated by the inhibition of IFN-y-regulated pathways (Figure 11C). The NF-KB complex was predicted to be inhibited in WT microglia after myelin treatment (bias-corrected $z$-score $-0.231, p$-value of overlap 3.15E-02), and inhibited more strongly in myelin-treated Myd88- microglia (Figure 11C). Myelin debris activated the translocation of NF-KB into the nucleus of cultured WT microglia, but the effect was less prominent than lipopolysaccharide (LPS), as detected by immunocytochemistry for the p65 subunit ( $24 \mathrm{~h}$ treatment in serum-free TCC medium; $30 \mu \mathrm{g} / \mathrm{mL}$ myelin; $10 \mathrm{ng} / \mathrm{mL}$ LPS (Sigma, L4516); data not shown). The predicted inhibition of NF-KB complex might explain the less strong nuclear translocation induced by myelin debris than LPS in WT microglia. However, the nuclear translocation of $\mathrm{NF}-\mathrm{kB}$ did not seem to be abolished in $M y d 88^{-/-}$microglia (data not shown). The activation of NF-KB and MAP kinases was actually reported in Myd88 ${ }^{-/}$mice (Kawai et al., 1999), although the induction of cytokines and IFN-y was abrogated (Adachi et al., 1998). In the mechanistic network (Figure 11C), the predicted activation or inhibition of signaling molecules such as STATs should be validated carefully, because the activity depends on phosphorylation and translocation into the nucleus, but mass spectrometry measures the amount of peptides/proteins. It has been shown that the signaling of type I IFN receptor (IFN- $\alpha / \beta$ receptor) is not necessary for remyelination (Schmidt et al., 2009). The cuprizone-induced demyelinated lesions of IFN- $\beta$-knockout mice had more OPCs and faster remyelination than WT at an early phase of remyelination but became similar later (Trebst et al., 2007). The microglia activation phenotypes in response to myelin debris might share some similarities with the "late response microglia" induced by type II IFN, which was identified by single-cell RNA-seq during the progression of neurodegeneration (Mathys et al., 2017). IFN-y was not identified in either the supernatant or the lysate of cultured microglia (Figure 8); it probably represents a node of gene expression regulated by various pathways, or it was expressed or secreted at a minute amount. Whether the IFN- $\gamma$-induced microglia phenotype is beneficial or harmful for regeneration is not clear. The predicted pathways will be analyzed in more details to generate hypotheses that can be tested by experiments. 
The pathways regulated by peroxisome proliferator-activated receptor $\alpha$ (PPAR- $\alpha$ ), PPAR$\delta$ and PPAR-y coactivator 1- $\alpha$ (Ppargc1a), which are involved in lipid metabolic processes (Moore and Tabas, 2011), were predicted by IPA to be activated in WT microglia after the exposure to myelin debris but less activated in $M y d 88^{-/}$microglia. The transcription factor activator protein 1 (AP-1, encoded by the Jun gene), which can be activated by MAP kinases downstream of MyD88 signaling, was predicted to be inhibited in myelin-treated WT microglia but less inhibited in Myd88-- microglia (Table 11). IPA did not identify any common pathways regulated by IRFs, which could also be downstream of MyD88 signaling, in both the comparisons of myelin-treated vs. control WT microglia and myelin treated $M y d 88^{-/}$vs. WT microglia.

In order to identify the activation phenotypes of microglia and the secreted molecules that are required for remyelination in vivo, we performed three RNA sequencing experiments of demyelinated lesions isolated from the spinal cord of WT and Myd88 ${ }^{-/}$mice. We reasoned that microglia / macrophages were the dominant cell types in the lesions at 7 $\mathrm{DPI}$, and therefore the gene expression profile of microglia / macrophages would contribute most to the transcriptome of the lesions. The expression of secreted molecules would also be reflected in the transcriptome. However, the analysis of the bulk RNA-seq of the lesions did not generate much insight due to the complex cell composition. In the first experiment, the spinal cords were sectioned using a vibratome, and the lesions were dissected using a blade at 2, 4, 7 and 21 DPI. This dissection method might have included variable amount of normal white matter in the samples, and the lesions might contain unknown proportions of each cell type at various time points. In the second experiment, microglia / macrophages were isolated from the lesions by MACS using anti-CD11b MicroBeads. To obtain enough material for RNA-seq, the lesions of two or three mice (four injection sites in each mouse) at $7 \mathrm{DPI}$ were pooled in one sample, resulting in a small sample size. In the third experiment, the lesions at 7 DPI were isolated accurately by laser microdissection. The differential gene expression analysis (Figure 12) and pathway analysis identified differences between the lesions of $M y d 88^{-/-}$and WT mice in many cellular processes and pathways, including inflammation, signaling, metabolism and so on. The transcriptomic data of lesions did not correlate well with the secreted proteins (such as Gdf15 and Timp1) identified in the supernatant of cultured microglia by proteomics, but further pathway analysis of the transcriptome of lesions might provide novel hypotheses about the molecular mechanisms of remyelination in vivo. Due to the complex cell composition in lesions, single-cell analysis would be a powerful method for 
identifying the activation phenotypes of microglia and other cells that are relevant to the stage of the disease or regeneration, even though the important subpopulations do not dominate in terms of cell number. Nevertheless, bulk RNA-seq of lesions have the advantage of intact tissue, because the generation of a single-cell suspension might affect gene expression (Espina et al., 2006).

We cannot exclude the possibility of Myd88 expression in cell types other than microglia / macrophages after injury in the CNS. However, since microglia predominantly express Myd88 in the CNS, impaired maturation of the phagosomes containing myelin debris in microglia might be one of the first steps that had defects in the lesions of $M y d 88^{-/}$mice during remyelination. The defects in phagosome maturation could affect the degradation of myelin debris and cause the accumulation of myelin debris in microglia. The signaling in microglia could also be disrupted by the lack of the adaptor protein MyD88. Consequently, microglia may not reach the appropriate activation states for promoting OPC proliferation, migration and differentiation, and/or creating an environment supportive for remyelination. The proteomic analyses of cultured microglia identified candidate molecules and pathways involved in the microglial response to purified myelin debris, but challenges remain to find the combination of molecules that is sufficient to promote remyelination. Furthermore, demyelinated lesions in a patient are heterogeneous - some lesions are in the phase of OPC recruitment, while others might require OPC differentiation (Franklin and Ffrench-Constant, 2017). After the molecular mechanisms of remyelination is unraveled by science, more effective therapy will still depend on the methods to determine which step of remyelination is limited in the patient.

\section{Validation of candidate molecules for the effects on OPC response}

The candidate molecules identified in the proteomic and transcriptomic analyses of cultured microglia and demyelinated lesions will be tested for their effects on OPC responses in tissue culture. The effect on OPC proliferation can be tested in OPC culture, using EdU to label proliferating cells (Figure S2). The OPC proliferation assay is being optimized in the OPC culture prepared by immunopanning (Watkins et al., 2008) using platelet-derived growth factor-AA (PDGF-AA) as a positive control (McKinnon et al., 1990; Raff et al., 1988). The effect on oligodendrocyte differentiation and myelination will be tested in organotypic hippocampal slice culture (OHSC) using 3,3',5-triiodo-L-thyronine (T3) as a positive control (Figure 13). 


\section{Lack of remyelination in organotypic hippocampal slice culture}

Ex vivo demyelination models were established for studying the mechanisms of remyelination and testing candidate molecules. Slice cultures are more accessible for experimentation than in vivo models, and the cell types and cellular architecture are better preserved than cell culture. Myelination develops in OHSC robustly (Figure 13), and the method to deplete and replenish microglia in OHSC provides a valuable tool for studying the roles of microglia in response to demyelination and during remyelination (Masuch et al., 2016). Lysolecithin-induced demyelination model has been established in organotypic cerebellar slice culture (OCSC) (Zhang et al., 2011). However, cerebellar slices become very thin and fragile in culture, making OCSC not ideal for the replenishment of exogenous microglia. Therefore, demyelination models were established in OHSC.

The injection of lysolecithin into organotypic hippocampal slices induced focal demyelination consistently, whereas the injection of PBS vehicle control left the myelin sheaths relatively intact. The treatment of OHSC with lysolecithin disrupted myelin only on the surface of the slices, but lysolecithin did not penetrate through the thick slices.

Treatment with higher concentrations of lysolecithin or for extended time caused extensive cell death. Therefore, a small amount of lysolecithin was injected into the slices to induce focal demyelination. However, remyelination was not observed 7 or 14 days after the injection of lysolecithin (Figure 14D and E). We speculated that lysolecithin led to cell death where it diffused to in the slices, resulting in insufficient OPCs for remyelination to occur. Therefore, an autoimmune demyelination model that selectively targets myelin and mature oligodendrocytes was established. Anti-MOG antibody and complement mediated demyelination in OHSC significantly (Figure 15A-C), but most axons remained unmyelinated 7 days after the treatment with anti-MOG antibody and complement was stopped (Figure 15E). The lack of remyelination in these two demyelination models might be due to the loss of regenerative capacity of the OPCs in hippocampal slices in culture, or regional differences of OPCs in terms of their remyelination capacity. The OPCs in organotypic cerebellar slice culture may be able to maintain their regenerative properties and perform remyelination (Zhang et al., 2011). If demyelination happens in the hippocampus, the progenitor cells might proliferate and migrate from the subventricular zone (SVZ) to the demyelinated lesion to perform remyelination. OPCs could be added to OHSC after demyelination for studying remyelination ex vivo (Zhang et al., 2011). 


\section{MOG antibody and complement-mediated demyelination in OHSC}

The treatment of myelinated OHSC with $10 \mu \mathrm{g} / \mathrm{mL}$ anti-MOG Z2 antibody and $5 \%$ guinea pig complement for $72 \mathrm{~h}$ induced demyelination (Figure $15 \mathrm{C}$ ). The $\sim 51 \%$ reduction of myelination index by Z2 antibody might be enlarged by increasing the concentration of complement without affecting the viability of OHSC. Anti-MOG antibody and complement mediated demyelination in the absence of T lymphocytes. Therefore, this model can be used to study the mechanisms of autoimmune demyelination and cellular responses to myelin damage without the complication of T cell infiltration in vivo.

In the OHSC depleted of microglia using clodronate (Kreutz et al., 2009; Masuch et al., 2016), anti-MOG Z2 antibody did not induce demyelination effectively compared with the isotype control (Figure 15F), which suggests the involvement of microglia in antibody and complement-mediated demyelination independent of T cells. Interestingly, Z2 antibody and complement did not induce demyelination in OHSC prepared from Myd88- mice as they did in WT OHSC (Figure 15G). This suggests that the antibody and complementmediated phagocytosis of (opsonized) myelin might require MyD88-dependent activation of microglia. After the exposure to myelin debris, cultured $M y d 88^{-/-}$microglia expressed lower levels of IgG Fc receptor I (high affinity immunoglobulin gamma Fc receptor I; encoded by the Fcgr1 gene, an ortholog of the human FCGR1A gene) than WT microglia, as analyzed by proteomics and IPA (Figure 11B). The IgG Fc receptor I on the surface of microglia might bind to the Fc part of anti-MOG $\operatorname{lgG}$, activating the phagocytosis of opsonized myelin. The preliminary results need validation by independent experiments, but they suggest a potential role of microglia in autoimmune demyelination by phagocytosing opsonized myelin. In contrast, microglia may regulate inflammatory response and secrete signals to guide remyelination in the toxin-induced demyelination model. Thus, the pathological context and specific activation phenotypes of microglia may determine the effector functions of microglia and whether the outcome of microglia activation is disease progression or regeneration. 


\section{The MyD88-dependent recruitment of OPCs may be specific to remyelination in the adult CNS}

It has been observed that a large number of activated microglia are present in the developing forebrain SVZ and reaches maximum in the early postnatal weeks, which coincides with the initiation of myelination. The activated microglia might promote oligodendrocyte development by producing TNF- $\alpha$, IL-1 $1 \beta$, IL-6 and IFN- $\gamma$ (ShigemotoMogami et al., 2014). Amoeboid microglia expressing LAMP-2 have been shown to peak in the developing white matter at postnatal day (P) 7 (Hagemeyer et al., 2017). Therefore, we asked whether the recruitment of OPC for developmental myelination requires MyD88 signaling in microglia. The number of OPCs in the corpus callosum was similar in WT and Myd88/- mice at P7 (Figure 16), which suggests that the MyD88-dependent regulation of OPC number may not be a key mechanism during development. The responses of microglia to TLR activation have been shown to reorganize and mature during postnatal development (Scheffel et al., 2012), which might explain the reason why MyD88dependent recruitment of OPCs is necessary for remyelination in the adult CNS but dispensable for developmental myelination.

\section{References}

Adachi, O., Kawai, T., Takeda, K., Matsumoto, M., Tsutsui, H., Sakagami, M., Nakanishi, K., and Akira, S. (1998). Targeted disruption of the MyD88 gene results in loss of IL-1and IL-18-mediated function. Immunity 9, 143-150.

Ahn, M., Yang, W., Kim, H., Jin, J.K., Moon, C., and Shin, T. (2012).

Immunohistochemical study of arginase-1 in the spinal cords of Lewis rats with experimental autoimmune encephalomyelitis. Brain research 1453, 77-86.

Allen, L.A., and Aderem, A. (1996). Mechanisms of phagocytosis. Curr Opin Immunol 8, 36-40.

Allen, N.J., and Barres, B.A. (2009). Neuroscience: Glia - more than just brain glue. Nature 457, 675-677.

Almazan, G., Honegger, P., and Matthieu, J.M. (1985). Triiodothyronine stimulation of oligodendroglial differentiation and myelination. A developmental study. Developmental neuroscience $7,45-54$.

Ank, N., Iversen, M.B., Bartholdy, C., Staeheli, P., Hartmann, R., Jensen, U.B., DagnaesHansen, F., Thomsen, A.R., Chen, Z., Haugen, H., et al. (2008). An important role for type 
III interferon (IFN-lambda/IL-28) in TLR-induced antiviral activity. Journal of immunology $180,2474-2485$.

Arai, K., and Lo, E.H. (2009). An oligovascular niche: cerebral endothelial cells promote the survival and proliferation of oligodendrocyte precursor cells. The Journal of neuroscience : the official journal of the Society for Neuroscience 29, 4351-4355.

Arnett, H.A., Mason, J., Marino, M., Suzuki, K., Matsushima, G.K., and Ting, J.P. (2001). TNF alpha promotes proliferation of oligodendrocyte progenitors and remyelination. Nature neuroscience 4,1116-1122.

Arnett, H.A., Wang, Y., Matsushima, G.K., Suzuki, K., and Ting, J.P. (2003). Functional genomic analysis of remyelination reveals importance of inflammation in oligodendrocyte regeneration. The Journal of neuroscience : the official journal of the Society for Neuroscience 23, 9824-9832.

Baas, D., Bourbeau, D., Sarlieve, L.L., Ittel, M.E., Dussault, J.H., and Puymirat, J. (1997). Oligodendrocyte maturation and progenitor cell proliferation are independently regulated by thyroid hormone. Glia 19, 324-332.

Baumann, N., and Pham-Dinh, D. (2001). Biology of oligodendrocyte and myelin in the mammalian central nervous system. Physiological reviews 81, 871-927.

Beertsen, W., Willenborg, M., Everts, V., Zirogianni, A., Podschun, R., Schroder, B., Eskelinen, E.L., and Saftig, P. (2008). Impaired phagosomal maturation in neutrophils leads to periodontitis in lysosomal-associated membrane protein-2 knockout mice. Journal of immunology $180,475-482$.

Belle, M., Godefroy, D., Dominici, C., Heitz-Marchaland, C., Zelina, P., Hellal, F., Bradke, F., and Chedotal, A. (2014). A simple method for 3D analysis of immunolabeled axonal tracts in a transparent nervous system. Cell Rep 9, 1191-1201.

Bennett, M.L., Bennett, F.C., Liddelow, S.A., Ajami, B., Zamanian, J.L., Fernhoff, N.B., Mulinyawe, S.B., Bohlen, C.J., Adil, A., Tucker, A., et al. (2016). New tools for studying microglia in the mouse and human CNS. Proceedings of the National Academy of Sciences of the United States of America 113, E1738-1746.

Bercury, K.K., and Macklin, W.B. (2015). Dynamics and mechanisms of CNS myelination. Dev Cell 32, 447-458.

Blander, J.M., and Medzhitov, R. (2004). Regulation of phagosome maturation by signals from toll-like receptors. Science 304, 1014-1018.

Blocker, A., Severin, F.F., Burkhardt, J.K., Bingham, J.B., Yu, H., Olivo, J.C., Schroer, T.A., Hyman, A.A., and Griffiths, G. (1997). Molecular requirements for bi-directional movement of phagosomes along microtubules. The Journal of cell biology 137, 113-129. 
Bohlen, C.J., Bennett, F.C., Tucker, A.F., Collins, H.Y., Mulinyawe, S.B., and Barres, B.A. (2017). Diverse Requirements for Microglial Survival, Specification, and Function Revealed by Defined-Medium Cultures. Neuron 94, 759-773 e758.

Boyd, A., Zhang, H., and Williams, A. (2013). Insufficient OPC migration into demyelinated lesions is a cause of poor remyelination in MS and mouse models. Acta neuropathologica 125, 841-859.

Bradford, M.M. (1976). A rapid and sensitive method for the quantitation of microgram quantities of protein utilizing the principle of protein-dye binding. Analytical biochemistry 72, 248-254.

Brahmachari, S., Fung, Y.K., and Pahan, K. (2006). Induction of glial fibrillary acidic protein expression in astrocytes by nitric oxide. The Journal of neuroscience : the official journal of the Society for Neuroscience 26, 4930-4939.

Breinbauer, R., and Kohn, M. (2003). Azide-alkyne coupling: a powerful reaction for bioconjugate chemistry. Chembiochem : a European journal of chemical biology 4, 11471149.

Butovsky, O., Jedrychowski, M.P., Moore, C.S., Cialic, R., Lanser, A.J., Gabriely, G., Koeglsperger, T., Dake, B., Wu, P.M., Doykan, C.E., et al. (2014). Identification of a unique TGF-beta-dependent molecular and functional signature in microglia. Nature neuroscience $17,131-143$.

Buttgereit, A., Lelios, I., Yu, X., Vrohlings, M., Krakoski, N.R., Gautier, E.L., Nishinakamura, R., Becher, B., and Greter, M. (2016). Sall1 is a transcriptional regulator defining microglia identity and function. Nature immunology 17, 1397-1406.

Cantuti-Castelvetri, L., Fitzner, D., Bosch-Queralt, M., Weil, M.T., Su, M., Sen, P., Ruhwedel, T., Mitkovski, M., Trendelenburg, G., Lutjohann, D., et al. (2018). Defective cholesterol clearance limits remyelination in the aged central nervous system. Science 359, 684-688.

Capello, E., Voskuhl, R.R., McFarland, H.F., and Raine, C.S. (1997). Multiple sclerosis: re-expression of a developmental gene in chronic lesions correlates with remyelination. Annals of neurology 41, 797-805.

Caron, E., and Hall, A. (1998). Identification of two distinct mechanisms of phagocytosis controlled by different Rho GTPases. Science 282, 1717-1721.

Carpenter, A.E., Jones, T.R., Lamprecht, M.R., Clarke, C., Kang, I.H., Friman, O., Guertin, D.A., Chang, J.H., Lindquist, R.A., Moffat, J., et al. (2006). CellProfiler: image analysis software for identifying and quantifying cell phenotypes. Genome Biol 7, R100. 
Chrast, R., Saher, G., Nave, K.A., and Verheijen, M.H. (2011). Lipid metabolism in myelinating glial cells: lessons from human inherited disorders and mouse models. J Lipid Res 52, 419-434.

Compston, A., and Coles, A. (2008). Multiple sclerosis. Lancet 372, 1502-1517.

Crawford, A.H., Tripathi, R.B., Foerster, S., McKenzie, I., Kougioumtzidou, E., Grist, M., Richardson, W.D., and Franklin, R.J. (2016). Pre-Existing Mature Oligodendrocytes Do Not Contribute to Remyelination following Toxin-Induced Spinal Cord Demyelination. The American journal of pathology 186, 511-516.

Crocker, S.J., Whitmire, J.K., Frausto, R.F., Chertboonmuang, P., Soloway, P.D., Whitton, J.L., and Campbell, I.L. (2006). Persistent macrophage/microglial activation and myelin disruption after experimental autoimmune encephalomyelitis in tissue inhibitor of metalloproteinase-1-deficient mice. The American journal of pathology 169, 2104-2116. Davies, C.L., and Miron, V.E. (2018). Distinct origins, gene expression and function of microglia and monocyte-derived macrophages in CNS myelin injury and regeneration. Clinical immunology 189, 57-62.

Deguine, J., and Barton, G.M. (2014). MyD88: a central player in innate immune signaling. F1000Prime Rep 6, 97.

Derfuss, T., Parikh, K., Velhin, S., Braun, M., Mathey, E., Krumbholz, M., Kumpfel, T., Moldenhauer, A., Rader, C., Sonderegger, P., et al. (2009). Contactin-2/TAG-1-directed autoimmunity is identified in multiple sclerosis patients and mediates gray matter pathology in animals. Proceedings of the National Academy of Sciences of the United States of America 106, 8302-8307.

Dombrowski, Y., O'Hagan, T., Dittmer, M., Penalva, R., Mayoral, S.R., Bankhead, P., Fleville, S., Eleftheriadis, G., Zhao, C., Naughton, M., et al. (2017). Regulatory T cells promote myelin regeneration in the central nervous system. Nature neuroscience $20,674-$ 680.

Doring, A., Sloka, S., Lau, L., Mishra, M., van Minnen, J., Zhang, X., Kinniburgh, D., Rivest, S., and Yong, V.W. (2015). Stimulation of monocytes, macrophages, and microglia by amphotericin $B$ and macrophage colony-stimulating factor promotes remyelination. The Journal of neuroscience : the official journal of the Society for Neuroscience 35, 11361148.

Eden, E., Navon, R., Steinfeld, I., Lipson, D., and Yakhini, Z. (2009). GOrilla: a tool for discovery and visualization of enriched $\mathrm{GO}$ terms in ranked gene lists. BMC Bioinformatics 10, 48. 
Elliott, C., Lindner, M., Arthur, A., Brennan, K., Jarius, S., Hussey, J., Chan, A., Stroet, A., Olsson, T., Willison, H., et al. (2012). Functional identification of pathogenic autoantibody responses in patients with multiple sclerosis. Brain : a journal of neurology $135,1819-$ 1833.

Espina, V., Wulfkuhle, J.D., Calvert, V.S., VanMeter, A., Zhou, W., Coukos, G., Geho, D.H., Petricoin, E.F., 3rd, and Liotta, L.A. (2006). Laser-capture microdissection. Nature protocols $1,586-603$.

Etxeberria, A., Mangin, J.M., Aguirre, A., and Gallo, V. (2010). Adult-born SVZ progenitors receive transient synapses during remyelination in corpus callosum. Nature neuroscience 13, 287-289.

Fard, M.K., van der Meer, F., Sanchez, P., Cantuti-Castelvetri, L., Mandad, S., Jakel, S., Fornasiero, E.F., Schmitt, S., Ehrlich, M., Starost, L., et al. (2017). BCAS1 expression defines a population of early myelinating oligodendrocytes in multiple sclerosis lesions. Sci Transl Med 9.

Fledrich, R., Abdelaal, T., Rasch, L., Bansal, V., Schutza, V., Brugger, B., Luchtenborg, C., Prukop, T., Stenzel, J., Rahman, R.U., et al. (2018). Targeting myelin lipid metabolism as a potential therapeutic strategy in a model of CMT1A neuropathy. Nature communications 9, 3025 .

Franklin, R.J., ffrench-Constant, C., Edgar, J.M., and Smith, K.J. (2012). Neuroprotection and repair in multiple sclerosis. Nat Rev Neurol 8, 624-634.

Franklin, R.J.M., and Ffrench-Constant, C. (2017). Regenerating CNS myelin - from mechanisms to experimental medicines. Nature reviews Neuroscience 18, 753-769. Fyffe-Maricich, S.L., Schott, A., Karl, M., Krasno, J., and Miller, R.H. (2013). Signaling through ERK1/2 controls myelin thickness during myelin repair in the adult central nervous system. The Journal of neuroscience : the official journal of the Society for Neuroscience 33, 18402-18408.

Gaesser, J.M., and Fyffe-Maricich, S.L. (2016). Intracellular signaling pathway regulation of myelination and remyelination in the CNS. Experimental neurology 283, 501-511.

Gautier, H.O., Evans, K.A., Volbracht, K., James, R., Sitnikov, S., Lundgaard, I., James, F., Lao-Peregrin, C., Reynolds, R., Franklin, R.J., et al. (2015). Neuronal activity regulates remyelination via glutamate signalling to oligodendrocyte progenitors. Nature communications 6, 8518 .

Ginhoux, F., and Garel, S. (2018). The mysterious origins of microglia. Nature neuroscience 21, 897-899. 
Ginhoux, F., Greter, M., Leboeuf, M., Nandi, S., See, P., Gokhan, S., Mehler, M.F., Conway, S.J., Ng, L.G., Stanley, E.R., et al. (2010). Fate mapping analysis reveals that adult microglia derive from primitive macrophages. Science 330, 841-845.

Gosselin, D., Skola, D., Coufal, N.G., Holtman, I.R., Schlachetzki, J.C.M., Sajti, E., Jaeger, B.N., O'Connor, C., Fitzpatrick, C., Pasillas, M.P., et al. (2017). An environmentdependent transcriptional network specifies human microglia identity. Science 356.

Hagemeyer, N., Hanft, K.M., Akriditou, M.A., Unger, N., Park, E.S., Stanley, E.R., Staszewski, O., Dimou, L., and Prinz, M. (2017). Microglia contribute to normal myelinogenesis and to oligodendrocyte progenitor maintenance during adulthood. Acta neuropathologica 134, 441-458.

Hall, A., Giese, N.A., and Richardson, W.D. (1996). Spinal cord oligodendrocytes develop from ventrally derived progenitor cells that express PDGF alpha-receptors. Development 122, 4085-4094.

Hammond, T.R., Gadea, A., Dupree, J., Kerninon, C., Nait-Oumesmar, B., Aguirre, A., and Gallo, V. (2014). Astrocyte-derived endothelin-1 inhibits remyelination through notch activation. Neuron $81,588-602$.

Hammond, T.R., Robinton, D., and Stevens, B. (2018). Microglia and the Brain: Complementary Partners in Development and Disease. Annu Rev Cell Dev Biol. Hanisch, U.K., and Kettenmann, H. (2007). Microglia: active sensor and versatile effector cells in the normal and pathologic brain. Nature neuroscience 10,1387-1394.

He, D., Marie, C., Zhao, C., Kim, B., Wang, J., Deng, Y., Clavairoly, A., Frah, M., Wang, H., He, X., et al. (2016). Chd7 cooperates with Sox10 and regulates the onset of CNS myelination and remyelination. Nature neuroscience 19,678-689.

Henneke, P., Takeuchi, O., Malley, R., Lien, E., Ingalls, R.R., Freeman, M.W., Mayadas, T., Nizet, V., Akira, S., Kasper, D.L., et al. (2002). Cellular activation, phagocytosis, and bactericidal activity against group $B$ streptococcus involve parallel myeloid differentiation factor 88-dependent and independent signaling pathways. Journal of immunology 169 , 3970-3977.

Henson, P.M., Bratton, D.L., and Fadok, V.A. (2001). The phosphatidylserine receptor: a crucial molecular switch? Nat Rev Mol Cell Biol 2, 627-633.

Hickman, S.E., Kingery, N.D., Ohsumi, T.K., Borowsky, M.L., Wang, L.C., Means, T.K., and El Khoury, J. (2013). The microglial sensome revealed by direct RNA sequencing. Nature neuroscience 16, 1896-1905. 
Hill, R.A., Medved, J., Patel, K.D., and Nishiyama, A. (2014). Organotypic slice cultures to study oligodendrocyte dynamics and myelination. Journal of visualized experiments : JoVE, e51835.

Horan, P.K., Melnicoff, M.J., Jensen, B.D., and Slezak, S.E. (1990). Fluorescent cell labeling for in vivo and in vitro cell tracking. Methods in cell biology 33, 469-490.

Hoyos, H.C., Rinaldi, M., Mendez-Huergo, S.P., Marder, M., Rabinovich, G.A., Pasquini, J.M., and Pasquini, L.A. (2014). Galectin-3 controls the response of microglial cells to limit cuprizone-induced demyelination. Neurobiol Dis 62, 441-455.

Huang, J.K., Jarjour, A.A., Nait Oumesmar, B., Kerninon, C., Williams, A., Krezel, W., Kagechika, H., Bauer, J., Zhao, C., Baron-Van Evercooren, A., et al. (2011). Retinoid X receptor gamma signaling accelerates CNS remyelination. Nature neuroscience $14,45-$ 53.

Huynh, K.K., Eskelinen, E.L., Scott, C.C., Malevanets, A., Saftig, P., and Grinstein, S. (2007). LAMP proteins are required for fusion of lysosomes with phagosomes. EMBO J 26, 313-324.

Huynh, K.K., Gershenzon, E., and Grinstein, S. (2008). Cholesterol accumulation by macrophages impairs phagosome maturation. The Journal of biological chemistry 283 , 35745-35755.

Imai, Y., Ibata, I., Ito, D., Ohsawa, K., and Kohsaka, S. (1996). A novel gene iba1 in the major histocompatibility complex class III region encoding an EF hand protein expressed in a monocytic lineage. Biochemical and biophysical research communications $224,855-$ 862.

Imai, Y., and Kohsaka, S. (2002). Intracellular signaling in M-CSF-induced microglia activation: role of Iba1. Glia 40,164-174.

International Multiple Sclerosis Genetics, C., Wellcome Trust Case Control, C., Sawcer, S., Hellenthal, G., Pirinen, M., Spencer, C.C., Patsopoulos, N.A., Moutsianas, L., Dilthey, A., Su, Z., et al. (2011). Genetic risk and a primary role for cell-mediated immune mechanisms in multiple sclerosis. Nature 476, 214-219.

Ishii, A., Fyffe-Maricich, S.L., Furusho, M., Miller, R.H., and Bansal, R. (2012). ERK1/ERK2 MAPK signaling is required to increase myelin thickness independent of oligodendrocyte differentiation and initiation of myelination. The Journal of neuroscience : the official journal of the Society for Neuroscience 32, 8855-8864.

Jarjour, A.A., Boyd, A., Dow, L.E., Holloway, R.K., Goebbels, S., Humbert, P.O., Williams, A., and ffrench-Constant, C. (2015). The polarity protein Scribble regulates myelination and remyelination in the central nervous system. PLoS Biol 13, e1002107. 
Jeffery, N.D., and Blakemore, W.F. (1995). Remyelination of mouse spinal cord axons demyelinated by local injection of lysolecithin. J Neurocytol 24, 775-781.

Jones, S.A., Jolson, D.M., Cuta, K.K., Mariash, C.N., and Anderson, G.W. (2003). Triiodothyronine is a survival factor for developing oligodendrocytes. Molecular and cellular endocrinology 199, 49-60.

Karin, M., and Clevers, H. (2016). Reparative inflammation takes charge of tissue regeneration. Nature 529, 307-315.

Kawai, T., Adachi, O., Ogawa, T., Takeda, K., and Akira, S. (1999). Unresponsiveness of MyD88-deficient mice to endotoxin. Immunity 11, 115-122.

Kawai, T., and Akira, S. (2006). TLR signaling. Cell Death Differ 13, 816-825.

Kazanis, I., Evans, K.A., Andreopoulou, E., Dimitriou, C., Koutsakis, C., Karadottir, R.T., and Franklin, R.J.M. (2017). Subependymal Zone-Derived Oligodendroblasts Respond to Focal Demyelination but Fail to Generate Myelin in Young and Aged Mice. Stem Cell Reports 8, 685-700.

Keren-Shaul, H., Spinrad, A., Weiner, A., Matcovitch-Natan, O., Dvir-Szternfeld, R., Ulland, T.K., David, E., Baruch, K., Lara-Astaiso, D., Toth, B., et al. (2017). A Unique Microglia Type Associated with Restricting Development of Alzheimer's Disease. Cell 169, 1276-1290 e1217.

Kinchen, J.M., and Ravichandran, K.S. (2008). Phagosome maturation: going through the acid test. Nat Rev Mol Cell Biol 9, 781-795.

Kluver, H., and Barrera, E. (1953). A method for the combined staining of cells and fibers in the nervous system. Journal of neuropathology and experimental neurology $12,400-$ 403.

Kotter, M.R., Li, W.W., Zhao, C., and Franklin, R.J. (2006). Myelin impairs CNS remyelination by inhibiting oligodendrocyte precursor cell differentiation. The Journal of neuroscience : the official journal of the Society for Neuroscience 26, 328-332.

Kotter, M.R., Setzu, A., Sim, F.J., Van Rooijen, N., and Franklin, R.J. (2001). Macrophage depletion impairs oligodendrocyte remyelination following lysolecithin-induced demyelination. Glia 35, 204-212.

Kramer, A., Green, J., Pollard, J., Jr., and Tugendreich, S. (2014). Causal analysis approaches in Ingenuity Pathway Analysis. Bioinformatics 30, 523-530.

Krasemann, S., Madore, C., Cialic, R., Baufeld, C., Calcagno, N., El Fatimy, R., Beckers, L., O'Loughlin, E., Xu, Y., Fanek, Z., et al. (2017). The TREM2-APOE Pathway Drives the Transcriptional Phenotype of Dysfunctional Microglia in Neurodegenerative Diseases. Immunity 47, 566-581 e569. 
Kremer, D., Gottle, P., Hartung, H.P., and Kury, P. (2016). Pushing Forward:

Remyelination as the New Frontier in CNS Diseases. Trends Neurosci 39, 246-263.

Kreutz, S., Koch, M., Bottger, C., Ghadban, C., Korf, H.W., and Dehghani, F. (2009). 2-

Arachidonoylglycerol elicits neuroprotective effects on excitotoxically lesioned dentate gyrus granule cells via abnormal-cannabidiol-sensitive receptors on microglial cells. Glia 57, 286-294.

Lampron, A., Larochelle, A., Laflamme, N., Prefontaine, P., Plante, M.M., Sanchez, M.G., Yong, V.W., Stys, P.K., Tremblay, M.E., and Rivest, S. (2015). Inefficient clearance of myelin debris by microglia impairs remyelinating processes. J Exp Med 212, 481-495.

Lang, J., Maeda, Y., Bannerman, P., Xu, J., Horiuchi, M., Pleasure, D., and Guo, F. (2013). Adenomatous polyposis coli regulates oligodendroglial development. The Journal of neuroscience : the official journal of the Society for Neuroscience 33, 3113-3130.

Levin, R., Grinstein, S., and Canton, J. (2016). The life cycle of phagosomes: formation, maturation, and resolution. Immunological reviews 273, 156-179.

Liddelow, S.A., Guttenplan, K.A., Clarke, L.E., Bennett, F.C., Bohlen, C.J., Schirmer, L., Bennett, M.L., Munch, A.E., Chung, W.S., Peterson, T.C., et al. (2017). Neurotoxic reactive astrocytes are induced by activated microglia. Nature 541, 481-487.

Linington, C., Bradl, M., Lassmann, H., Brunner, C., and Vass, K. (1988). Augmentation of demyelination in rat acute allergic encephalomyelitis by circulating mouse monoclonal antibodies directed against a myelin/oligodendrocyte glycoprotein. The American journal of pathology $130,443-454$.

Linnington, C., Webb, M., and Woodhams, P.L. (1984). A novel myelin-associated glycoprotein defined by a mouse monoclonal antibody. Journal of neuroimmunology 6 , 387-396.

Liu, N., Montgomery, R.R., Barthold, S.W., and Bockenstedt, L.K. (2004). Myeloid differentiation antigen 88 deficiency impairs pathogen clearance but does not alter inflammation in Borrelia burgdorferi-infected mice. Infect Immun 72, 3195-3203.

Lucchinetti, C., Bruck, W., Parisi, J., Scheithauer, B., Rodriguez, M., and Lassmann, H. (1999). A quantitative analysis of oligodendrocytes in multiple sclerosis lesions. A study of 113 cases. Brain : a journal of neurology 122 ( Pt 12), 2279-2295.

Ludwin, S.K. (2000). Understanding multiple sclerosis: lessons from pathology. Annals of neurology 47, 691-693.

Ludwin, S.K., and Sternberger, N.H. (1984). An immunohistochemical study of myelin proteins during remyelination in the central nervous system. Acta neuropathologica 63 , 240-248. 
Lukacs, G.L., Rotstein, O.D., and Grinstein, S. (1990). Phagosomal acidification is mediated by a vacuolar-type $\mathrm{H}(+)$-ATPase in murine macrophages. The Journal of biological chemistry 265, 21099-21107.

Ma, Y.J., Hein, E., Munthe-Fog, L., Skjoedt, M.O., Bayarri-Olmos, R., Romani, L., and Garred, P. (2015). Soluble Collectin-12 (CL-12) Is a Pattern Recognition Molecule Initiating Complement Activation via the Alternative Pathway. Journal of immunology 195, 3365-3373.

Machado, V., Haas, S.J., von Bohlen Und Halbach, O., Wree, A., Krieglstein, K., Unsicker, K., and Spittau, B. (2016). Growth/differentiation factor-15 deficiency compromises dopaminergic neuron survival and microglial response in the 6-hydroxydopamine mouse model of Parkinson's disease. Neurobiol Dis 88, 1-15.

Madsen, P.M., Motti, D., Karmally, S., Szymkowski, D.E., Lambertsen, K.L., Bethea, J.R., and Brambilla, R. (2016). Oligodendroglial TNFR2 Mediates Membrane TNF-Dependent Repair in Experimental Autoimmune Encephalomyelitis by Promoting Oligodendrocyte Differentiation and Remyelination. The Journal of neuroscience : the official journal of the Society for Neuroscience 36, 5128-5143.

Marr, K.A., Balajee, S.A., Hawn, T.R., Ozinsky, A., Pham, U., Akira, S., Aderem, A., and Liles, W.C. (2003). Differential role of MyD88 in macrophage-mediated responses to opportunistic fungal pathogens. Infect Immun 71, 5280-5286.

Mason, J.L., Suzuki, K., Chaplin, D.D., and Matsushima, G.K. (2001). Interleukin-1beta promotes repair of the CNS. The Journal of neuroscience : the official journal of the Society for Neuroscience 21, 7046-7052.

Mason, J.L., Xuan, S., Dragatsis, I., Efstratiadis, A., and Goldman, J.E. (2003). Insulin-like growth factor (IGF) signaling through type 1 IGF receptor plays an important role in remyelination. The Journal of neuroscience : the official journal of the Society for Neuroscience 23, 7710-7718.

Masuch, A., van der Pijl, R., Funer, L., Wolf, Y., Eggen, B., Boddeke, E., and Biber, K. (2016). Microglia replenished OHSC: A culture system to study in vivo like adult microglia. Glia 64, 1285-1297.

Mathys, H., Adaikkan, C., Gao, F., Young, J.Z., Manet, E., Hemberg, M., De Jager, P.L., Ransohoff, R.M., Regev, A., and Tsai, L.H. (2017). Temporal Tracking of Microglia Activation in Neurodegeneration at Single-Cell Resolution. Cell Rep 21, 366-380. McKinnon, R.D., Matsui, T., Dubois-Dalcq, M., and Aaronson, S.A. (1990). FGF modulates the PDGF-driven pathway of oligodendrocyte development. Neuron 5, 603614. 
Mi, S., Miller, R.H., Lee, X., Scott, M.L., Shulag-Morskaya, S., Shao, Z., Chang, J., Thill, G., Levesque, M., Zhang, M., et al. (2005). LINGO-1 negatively regulates myelination by oligodendrocytes. Nature neuroscience 8, 745-751.

Mi, S., Miller, R.H., Tang, W., Lee, X., Hu, B., Wu, W., Zhang, Y., Shields, C.B., Zhang, Y., Miklasz, S., et al. (2009). Promotion of central nervous system remyelination by induced differentiation of oligodendrocyte precursor cells. Annals of neurology 65, 304315.

Michel, K., Zhao, T., Karl, M., Lewis, K., and Fyffe-Maricich, S.L. (2015). Translational control of myelin basic protein expression by ERK2 MAP kinase regulates timely remyelination in the adult brain. The Journal of neuroscience : the official journal of the Society for Neuroscience 35, 7850-7865.

Miron, V.E. (2017). Microglia-driven regulation of oligodendrocyte lineage cells, myelination, and remyelination. Journal of leukocyte biology 101, 1103-1108.

Miron, V.E., Boyd, A., Zhao, J.W., Yuen, T.J., Ruckh, J.M., Shadrach, J.L., van Wijngaarden, P., Wagers, A.J., Williams, A., Franklin, R.J.M., et al. (2013). M2 microglia and macrophages drive oligodendrocyte differentiation during CNS remyelination. Nature neuroscience 16, 1211-1218.

Monsma, P.C., and Brown, A. (2012). FluoroMyelin Red is a bright, photostable and nontoxic fluorescent stain for live imaging of myelin. J Neurosci Methods 209, 344-350.

Moore, C.S., Milner, R., Nishiyama, A., Frausto, R.F., Serwanski, D.R., Pagarigan, R.R., Whitton, J.L., Miller, R.H., and Crocker, S.J. (2011). Astrocytic tissue inhibitor of metalloproteinase-1 (TIMP-1) promotes oligodendrocyte differentiation and enhances CNS myelination. The Journal of neuroscience : the official journal of the Society for Neuroscience 31, 6247-6254.

Moore, K.J., and Tabas, I. (2011). Macrophages in the pathogenesis of atherosclerosis. Cell 145, 341-355.

Morell, P., Barrett, C.V., Mason, J.L., Toews, A.D., Hostettler, J.D., Knapp, G.W., and Matsushima, G.K. (1998). Gene expression in brain during cuprizone-induced demyelination and remyelination. Molecular and cellular neurosciences 12, 220-227. Moyon, S., Dubessy, A.L., Aigrot, M.S., Trotter, M., Huang, J.K., Dauphinot, L., Potier, M.C., Kerninon, C., Melik Parsadaniantz, S., Franklin, R.J., et al. (2015). Demyelination causes adult CNS progenitors to revert to an immature state and express immune cues that support their migration. The Journal of neuroscience : the official journal of the Society for Neuroscience 35, 4-20. 
Najm, F.J., Madhavan, M., Zaremba, A., Shick, E., Karl, R.T., Factor, D.C., Miller, T.E., Nevin, Z.S., Kantor, C., Sargent, A., et al. (2015). Drug-based modulation of endogenous stem cells promotes functional remyelination in vivo. Nature 522, 216-220.

Nakatani, H., Martin, E., Hassani, H., Clavairoly, A., Maire, C.L., Viadieu, A., Kerninon, C., Delmasure, A., Frah, M., Weber, M., et al. (2013). Ascl1/Mash1 promotes brain oligodendrogenesis during myelination and remyelination. The Journal of neuroscience : the official journal of the Society for Neuroscience 33, 9752-9768.

Nave, K.A. (2010). Myelination and the trophic support of long axons. Nature reviews Neuroscience 11, 275-283.

Nave, K.A., Lai, C., Bloom, F.E., and Milner, R.J. (1987). Splice site selection in the proteolipid protein (PLP) gene transcript and primary structure of the DM-20 protein of central nervous system myelin. Proceedings of the National Academy of Sciences of the United States of America 84, 5665-5669.

Nichterwitz, S., Chen, G., Aguila Benitez, J., Yilmaz, M., Storvall, H., Cao, M., Sandberg, R., Deng, Q., and Hedlund, E. (2016). Laser capture microscopy coupled with Smart-seq2 for precise spatial transcriptomic profiling. Nature communications 7, 12139 .

Nishiyama, A., Lin, X.H., Giese, N., Heldin, C.H., and Stallcup, W.B. (1996). Colocalization of NG2 proteoglycan and PDGF alpha-receptor on O2A progenitor cells in the developing rat brain. Journal of neuroscience research 43, 299-314.

O'Brien, J.S., and Sampson, E.L. (1965). Lipid composition of the normal human brain: gray matter, white matter, and myelin. J Lipid Res 6, 537-544.

Olah, M., Amor, S., Brouwer, N., Vinet, J., Eggen, B., Biber, K., and Boddeke, H.W. (2012). Identification of a microglia phenotype supportive of remyelination. Glia 60, 306321.

Onoguchi, K., Yoneyama, M., Takemura, A., Akira, S., Taniguchi, T., Namiki, H., and Fujita, T. (2007). Viral infections activate types I and III interferon genes through a common mechanism. The Journal of biological chemistry 282, 7576-7581.

Pagenstecher, A., Stalder, A.K., Kincaid, C.L., Shapiro, S.D., and Campbell, I.L. (1998).

Differential expression of matrix metalloproteinase and tissue inhibitor of matrix metalloproteinase genes in the mouse central nervous system in normal and inflammatory states. The American journal of pathology 152, 729-741.

Pasquini, L.A., Millet, V., Hoyos, H.C., Giannoni, J.P., Croci, D.O., Marder, M., Liu, F.T., Rabinovich, G.A., and Pasquini, J.M. (2011). Galectin-3 drives oligodendrocyte differentiation to control myelin integrity and function. Cell Death Differ 18, 1746-1756. 
Patel, J.R., McCandless, E.E., Dorsey, D., and Klein, R.S. (2010). CXCR4 promotes differentiation of oligodendrocyte progenitors and remyelination. Proceedings of the National Academy of Sciences of the United States of America 107, 11062-11067. Pauwels, A.M., Trost, M., Beyaert, R., and Hoffmann, E. (2017). Patterns, Receptors, and Signals: Regulation of Phagosome Maturation. Trends Immunol 38, 407-422.

Perry, V.H. (1998). A revised view of the central nervous system microenvironment and major histocompatibility complex class II antigen presentation. Journal of neuroimmunology 90,113-121.

Petkovic, F., Campbell, I.L., Gonzalez, B., and Castellano, B. (2016). Astrocyte-targeted production of interleukin- 6 reduces astroglial and microglial activation in the cuprizone demyelination model: Implications for myelin clearance and oligodendrocyte maturation. Glia 64, 2104-2119.

Pham-Dinh, D., Mattei, M.G., Nussbaum, J.L., Roussel, G., Pontarotti, P., Roeckel, N., Mather, I.H., Artzt, K., Lindahl, K.F., and Dautigny, A. (1993). Myelin/oligodendrocyte glycoprotein is a member of a subset of the immunoglobulin superfamily encoded within the major histocompatibility complex. Proceedings of the National Academy of Sciences of the United States of America 90, 7990-7994.

Pineau, I., Sun, L., Bastien, D., and Lacroix, S. (2010). Astrocytes initiate inflammation in the injured mouse spinal cord by promoting the entry of neutrophils and inflammatory monocytes in an IL-1 receptor/MyD88-dependent fashion. Brain Behav Immun 24, 540553.

Prineas, J.W., Barnard, R.O., Kwon, E.E., Sharer, L.R., and Cho, E.S. (1993). Multiple sclerosis: remyelination of nascent lesions. Annals of neurology 33, 137-151.

Prineas, J.W., Kwon, E.E., Goldenberg, P.Z., Ilyas, A.A., Quarles, R.H., Benjamins, J.A., and Sprinkle, T.J. (1989). Multiple sclerosis. Oligodendrocyte proliferation and differentiation in fresh lesions. Laboratory investigation; a journal of technical methods and pathology 61, 489-503.

Raff, M.C., Lillien, L.E., Richardson, W.D., Burne, J.F., and Noble, M.D. (1988). Plateletderived growth factor from astrocytes drives the clock that times oligodendrocyte development in culture. Nature 333, 562-565.

Raine, C.S., and Wu, E. (1993). Multiple sclerosis: remyelination in acute lesions. Journal of neuropathology and experimental neurology 52, 199-204.

Ransohoff, R.M. (2016). A polarizing question: do M1 and M2 microglia exist? Nature neuroscience 19, 987-991. 
Ransohoff, R.M., and Perry, V.H. (2009). Microglial physiology: unique stimuli, specialized responses. Annu Rev Immunol 27, 119-145.

Reynolds, R., and Wilkin, G.P. (1988). Development of macroglial cells in rat cerebellum. II. An in situ immunohistochemical study of oligodendroglial lineage from precursor to mature myelinating cell. Development 102, 409-425.

Rostovtsev, V.V., Green, L.G., Fokin, V.V., and Sharpless, K.B. (2002). A stepwise huisgen cycloaddition process: copper(I)-catalyzed regioselective "ligation" of azides and terminal alkynes. Angewandte Chemie 41, 2596-2599.

Roth, P., Junker, M., Tritschler, I., Mittelbronn, M., Dombrowski, Y., Breit, S.N., Tabatabai, G., Wick, W., Weller, M., and Wischhusen, J. (2010). GDF-15 contributes to proliferation and immune escape of malignant gliomas. Clin Cancer Res 16, 3851-3859.

Rotshenker, S. (2009). The role of Galectin-3/MAC-2 in the activation of the innateimmune function of phagocytosis in microglia in injury and disease. Journal of molecular neuroscience : MN 39, 99-103.

Salic, A., and Mitchison, T.J. (2008). A chemical method for fast and sensitive detection of DNA synthesis in vivo. Proceedings of the National Academy of Sciences of the United States of America 105, 2415-2420.

Samanta, J., Grund, E.M., Silva, H.M., Lafaille, J.J., Fishell, G., and Salzer, J.L. (2015). Inhibition of Gli1 mobilizes endogenous neural stem cells for remyelination. Nature 526, 448-452.

Satoh, J., Kino, Y., Asahina, N., Takitani, M., Miyoshi, J., Ishida, T., and Saito, Y. (2016). TMEM119 marks a subset of microglia in the human brain. Neuropathology : official journal of the Japanese Society of Neuropathology 36, 39-49.

Savina, A., and Amigorena, S. (2007). Phagocytosis and antigen presentation in dendritic cells. Immunological reviews 219, 143-156.

Savina, A., Jancic, C., Hugues, S., Guermonprez, P., Vargas, P., Moura, I.C., LennonDumenil, A.M., Seabra, M.C., Raposo, G., and Amigorena, S. (2006). NOX2 controls phagosomal $\mathrm{pH}$ to regulate antigen processing during crosspresentation by dendritic cells. Cell 126, 205-218.

Scheffel, J., Regen, T., Van Rossum, D., Seifert, S., Ribes, S., Nau, R., Parsa, R., Harris, R.A., Boddeke, H.W., Chuang, H.N., et al. (2012). Toll-like receptor activation reveals developmental reorganization and unmasks responder subsets of microglia. Glia 60 , 1930-1943. 
Scheffler, J.M., Schiefermeier, N., and Huber, L.A. (2014). Mild fixation and permeabilization protocol for preserving structures of endosomes, focal adhesions, and actin filaments during immunofluorescence analysis. Methods Enzymol 535, 93-102. Schindelin, J., Arganda-Carreras, I., Frise, E., Kaynig, V., Longair, M., Pietzsch, T., Preibisch, S., Rueden, C., Saalfeld, S., Schmid, B., et al. (2012). Fiji: an open-source platform for biological-image analysis. Nature methods 9, 676-682.

Schmidt, H., Raasch, J., Merkler, D., Klinker, F., Krauss, S., Bruck, W., and Prinz, M. (2009). Type I interferon receptor signalling is induced during demyelination while its function for myelin damage and repair is redundant. Experimental neurology 216, 306311.

Shigemoto-Mogami, Y., Hoshikawa, K., Goldman, J.E., Sekino, Y., and Sato, K. (2014). Microglia enhance neurogenesis and oligodendrogenesis in the early postnatal subventricular zone. The Journal of neuroscience : the official journal of the Society for Neuroscience 34, 2231-2243.

Sim, F.J., Hinks, G.L., and Franklin, R.J. (2000). The re-expression of the homeodomain transcription factor Gtx during remyelination of experimentally induced demyelinating lesions in young and old rat brain. Neuroscience 100, 131-139.

Snaidero, N., and Simons, M. (2014). Myelination at a glance. Journal of cell science 127, 2999-3004.

Sowell, E.R., Thompson, P.M., Holmes, C.J., Jernigan, T.L., and Toga, A.W. (1999). In vivo evidence for post-adolescent brain maturation in frontal and striatal regions. Nature neuroscience 2, 859-861.

Sowell, E.R., Thompson, P.M., Tessner, K.D., and Toga, A.W. (2001). Mapping continued brain growth and gray matter density reduction in dorsal frontal cortex: Inverse relationships during postadolescent brain maturation. The Journal of neuroscience : the official journal of the Society for Neuroscience 21, 8819-8829.

Sparrow, J.R. (1994). Inducible nitric oxide synthase in the central nervous system. Journal of molecular neuroscience : MN 5, 219-229.

Stach, C.M., Turnay, X., Voll, R.E., Kern, P.M., Kolowos, W., Beyer, T.D., Kalden, J.R., and Herrmann, M. (2000). Treatment with annexin V increases immunogenicity of apoptotic human T-cells in Balb/c mice. Cell Death Differ 7, 911-915.

Steelman, A.J., Zhou, Y., Koito, H., Kim, S., Payne, H.R., Lu, Q.R., and Li, J. (2016). Activation of oligodendroglial Stat3 is required for efficient remyelination. Neurobiol Dis 91 , 336-346. 
Stoppini, L., Buchs, P.A., and Muller, D. (1991). A simple method for organotypic cultures of nervous tissue. J Neurosci Methods 37, 173-182.

Strelau, J., Bottner, M., Lingor, P., Suter-Crazzolara, C., Galter, D., Jaszai, J., Sullivan, A., Schober, A., Krieglstein, K., and Unsicker, K. (2000). GDF-15/MIC-1 a novel member of the TGF-beta superfamily. J Neural Transm Suppl, 273-276.

Swiss, V.A., Nguyen, T., Dugas, J., Ibrahim, A., Barres, B., Androulakis, I.P., and Casaccia, P. (2011). Identification of a gene regulatory network necessary for the initiation of oligodendrocyte differentiation. PloS one 6, e18088.

Tarassishin, L., Suh, H.S., and Lee, S.C. (2014). LPS and IL-1 differentially activate mouse and human astrocytes: role of CD14. Glia 62, 999-1013.

Toft-Hansen, H., Nuttall, R.K., Edwards, D.R., and Owens, T. (2004). Key metalloproteinases are expressed by specific cell types in experimental autoimmune encephalomyelitis. Journal of immunology 173, 5209-5218.

Town, T., Nikolic, V., and Tan, J. (2005). The microglial "activation" continuum: from innate to adaptive responses. Journal of neuroinflammation 2, 24.

Trebst, C., Heine, S., Lienenklaus, S., Lindner, M., Baumgartner, W., Weiss, S., and Stangel, M. (2007). Lack of interferon-beta leads to accelerated remyelination in a toxic model of central nervous system demyelination. Acta neuropathologica 114, 587-596. Ulrich, R., Gerhauser, I., Seeliger, F., Baumgartner, W., and Alldinger, S. (2005). Matrix metalloproteinases and their inhibitors in the developing mouse brain and spinal cord: a reverse transcription quantitative polymerase chain reaction study. Developmental neuroscience $27,408-418$.

Underhill, D.M., and Ozinsky, A. (2002). Phagocytosis of microbes: complexity in action. Annu Rev Immunol 20, 825-852.

Wallace, P.K., Tario, J.D., Jr., Fisher, J.L., Wallace, S.S., Ernstoff, M.S., and Muirhead, K.A. (2008). Tracking antigen-driven responses by flow cytometry: monitoring proliferation by dye dilution. Cytometry Part A : the journal of the International Society for Analytical Cytology 73, 1019-1034.

Wang, Q., Chan, T.R., Hilgraf, R., Fokin, V.V., Sharpless, K.B., and Finn, M.G. (2003). Bioconjugation by copper(I)-catalyzed azide-alkyne [3 + 2] cycloaddition. Journal of the American Chemical Society 125, 3192-3193.

Watkins, T.A., Emery, B., Mulinyawe, S., and Barres, B.A. (2008). Distinct stages of myelination regulated by gamma-secretase and astrocytes in a rapidly myelinating CNS coculture system. Neuron 60, 555-569. 
Wes, P.D., Holtman, I.R., Boddeke, E.W., Moller, T., and Eggen, B.J. (2016). Next generation transcriptomics and genomics elucidate biological complexity of microglia in health and disease. Glia 64, 197-213.

Xing, Y.L., Roth, P.T., Stratton, J.A., Chuang, B.H., Danne, J., Ellis, S.L., Ng, S.W., Kilpatrick, T.J., and Merson, T.D. (2014). Adult neural precursor cells from the subventricular zone contribute significantly to oligodendrocyte regeneration and remyelination. The Journal of neuroscience : the official journal of the Society for Neuroscience 34, 14128-14146.

Xu, X., Cai, J., Fu, H., Wu, R., Qi, Y., Modderman, G., Liu, R., and Qiu, M. (2000). Selective expression of Nkx-2.2 transcription factor in chicken oligodendrocyte progenitors and implications for the embryonic origin of oligodendrocytes. Molecular and cellular neurosciences $16,740-753$.

Yates, R.M., Hermetter, A., and Russell, D.G. (2005). The kinetics of phagosome maturation as a function of phagosome/lysosome fusion and acquisition of hydrolytic activity. Traffic 6, 413-420.

Yates, R.M., and Russell, D.G. (2005). Phagosome maturation proceeds independently of stimulation of toll-like receptors 2 and 4 . Immunity 23, 409-417.

Yuen, T.J., Johnson, K.R., Miron, V.E., Zhao, C., Quandt, J., Harrisingh, M.C., Swire, M., Williams, A., McFarland, H.F., Franklin, R.J., et al. (2013). Identification of endothelin 2 as an inflammatory factor that promotes central nervous system remyelination. Brain : a journal of neurology 136, 1035-1047.

Zeisel, A., Munoz-Manchado, A.B., Codeluppi, S., Lonnerberg, P., La Manno, G., Jureus, A., Marques, S., Munguba, H., He, L., Betsholtz, C., et al. (2015). Brain structure. Cell types in the mouse cortex and hippocampus revealed by single-cell RNA-seq. Science 347, 1138-1142.

Zhang, H., Jarjour, A.A., Boyd, A., and Williams, A. (2011). Central nervous system remyelination in culture--a tool for multiple sclerosis research. Experimental neurology 230, 138-148.

Zhang, Y., Chen, K., Sloan, S.A., Bennett, M.L., Scholze, A.R., O'Keeffe, S., Phatnani, H.P., Guarnieri, P., Caneda, C., Ruderisch, N., et al. (2014). An RNA-sequencing transcriptome and splicing database of glia, neurons, and vascular cells of the cerebral cortex. The Journal of neuroscience : the official journal of the Society for Neuroscience 34, 11929-11947. 
Zhou, Q., Choi, G., and Anderson, D.J. (2001). The bHLH transcription factor Olig2 promotes oligodendrocyte differentiation in collaboration with Nkx2.2. Neuron 31,791807. 


\section{Acknowledgements}

I would like to thank Prof. Dr. Mikael Simons for advice, guidance and support throughout my PhD;

Prof. Dr. Blanche Schwappach and Prof. Dr. Steven Johnsen for being members of the Thesis Advisory Committee and the examination board, and for feedback and comments on my PhD project;

Prof. Dr. André Fischer, Prof. Dr. Dr. Hannelore Ehrenreich and Prof. Dr. Tiago Fleming Outeiro for being members of the examination board;

Dr. Ludovico Cantuti-Castelvetri for teaching me a lot of techniques and methods, scientific discussions, support and help, including but not limited to: the stereotactic injections for histology and the first RNA sequencing experiment of lesions, perfusion, the preparation of semithin sections and methylene blue stain, the preparation of bone marrow-derived macrophage culture, the preparation of myelin, and coffee;

Dr. Ioannis Alexopoulos for the ImageJ macros (for the analysis of myelination and the volume of myelin debris in microglia / macrophages) and detailed advice on imaging and image analysis;

Dr. Paula Sanchez for teaching me cell culture techniques, ICC, imaging and image analysis; detailed protocol and advice on the preparation of postnatal mouse brains for IHC; advice and support;

Dr. Marie-Theres Weil for many protocols, help and advice;

Liliana Pedro for oligodendrocyte cultures, discussions and help;

Dr. David Brockelt for proofreading my PhD thesis;

Dr. Martina Schifferer and Kerstin Karg for electron microscopy;

Dr. Sebastian Timmler and Ulrich Weikert for some techniques and help;

Giselheid Schulz for preparing cell cultures in our laboratory in Göttingen;

Dr. Cordelia Imig for detailed advice on the establishment of organotypic hippocampal slice culture;

Dr. Dirk Fitzner for some techniques and advice;

Dr. Stephan Müller and Prof. Dr. Stefan Lichtenthaler for proteomics and Ingenuity Pathway Analysis;

Nirmal Raman Kannaiyan and Prof. Dr. Moritz Rossner for transcriptomics; 
Dr. Sven Wichert for very detailed advice and help for the establishment of laser microdissection;

Dr. Özgün Gökce for advice on laser microdissection;

Prof. Dr. Uwe-Karsten Hanisch and Dr. Nasrin Saiepour for Myd88

Dr. Arthur Liesz and Kerstin Thuß-Silczak for Myd88-/ mice in Munich;

Prof. Dr. Edgar Meinl and Heike Rübsamen for the anti-MOG antibodies.

I would also like to thank Dr. Steffen Burkhardt and Kerstin Grüniger for the support from the IMPRS for Molecular Biology Program; Prof. King L. Chow, Prof. Nancy Y. Ip and Prof. King-Wai Yau for mentoring.

Last but not least, I would like to thank my husband Haoqing, my parents and grandparents for their love and support. I also want to thank my friends for their support, which helped me to go through the difficult periods in $\mathrm{PhD}$ and still enjoy life and have hope for the future. 


\section{Supplemental material}

\section{Activation of cultured microglia / macrophages after the exposure to myelin debris}

The expression of several activation markers by cultured microglia or macrophages was checked after myelin treatment. Microglia were isolated by MACS and cultured in DMEM/FCS/L929 medium for 2 days, and then treated with myelin for $4 \mathrm{~h}$ in serum-free TCC medium. Most microglia expressed the activation marker iNOS, for instance (Figure S1C). Macrophage culture expressed activation markers such as galectin-3 and MHC class II after $24 \mathrm{~h}$ of exposure to myelin debris in DMEM/M-CSF medium (Figure S1A and B).

(A) lectin galectin-3

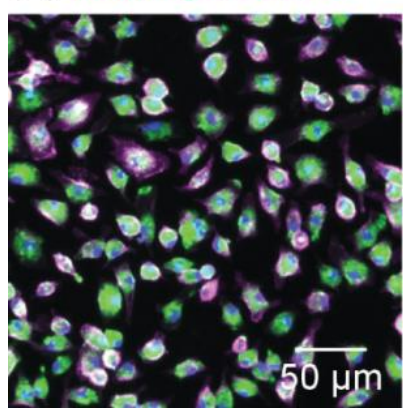

(B) lectin MHC class II

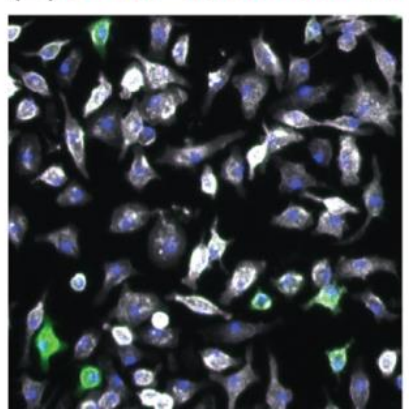

(C) lectin iNOS DAPI

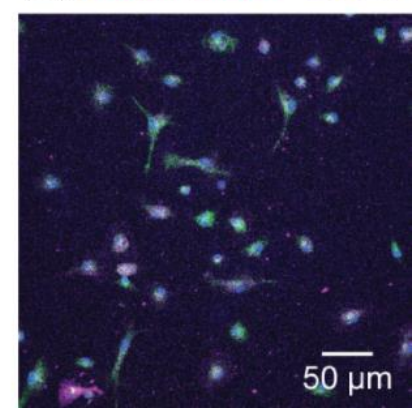

Figure S1. Cultured microglia and macrophages expressed activation markers after the exposure to purified myelin debris. After $24 \mathrm{~h}$ of treatment with myelin, macrophages (magenta) expressed (A) galectin-3 (green) and (B) MHC class II (green). (C) Microglia (magenta) expressed iNOS (green) after $4 \mathrm{~h}$ of treatment with myelin.

\section{OPC proliferation measured by EdU in tissue culture}

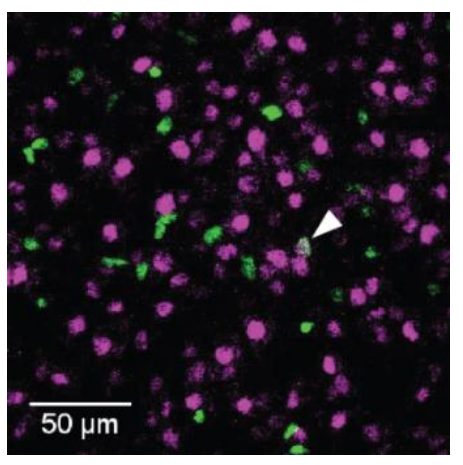

Figure S2. Proliferating cells were labeled with $10 \mu \mathrm{M}$ EdU for 2 days in OHSC. The co-labeling of EdU (green) and OLIG2 (magenta) identifies the nucleus of an oligodendrocyte-lineage cell that proliferated during the time of labeling. The same labeling method applies to cell culture. 


\section{Appendices}

Appendix 1. The proteins detected in the supernatant of microglia culture whose amount was significantly different in the two comparisons myelin_Myd88 ${ }^{--} /$ myelin_WT and myelin_WT / control WT $\left(\log _{2}(\right.$ LFQ ratio $) \leq-0.5$ or $\geq 0.5$, and $p$-value $\left.<0.05\right)$.

\begin{tabular}{|c|c|c|c|c|c|c|c|c|c|c|c|c|c|}
\hline & & \multicolumn{4}{|c|}{$\log _{2}$ LFQ fold changes } & \multicolumn{4}{|c|}{ t-test $p$-values } & \multicolumn{4}{|c|}{$\begin{array}{l}\text { Permutation based FDR correction } \\
\text { (significant: }+ \text { ) }\end{array}$} \\
\hline Protein names & $\begin{array}{l}\text { Gene } \\
\text { names }\end{array}$ & $\begin{array}{l}\log _{2} \\
\left(\mathrm{KO} \_\mathrm{M} /\right. \\
\left.\mathrm{KO} \_\mathrm{C}\right)\end{array}$ & $\begin{array}{l}\log _{2} \\
\left(\mathrm{WT} \_\mathrm{M} /\right. \\
\text { WT_C) }\end{array}$ & $\begin{array}{l}\log _{2} \\
\left(\mathrm{KO} \_\mathrm{Cl}\right. \\
\mathrm{WT} C \mathrm{C})\end{array}$ & $\begin{array}{l}\log _{2} \\
\left(\mathrm{KO} \_\mathrm{M} /\right. \\
\text { WT_M) }\end{array}$ & $\begin{array}{l}\mathrm{p} \\
\left(\mathrm{KO} \mathrm{K}_{-}\right. \\
\mathrm{M} / \mathrm{KO} \\
\left.\mathrm{C}_{\mathrm{C}}\right)\end{array}$ & $\begin{array}{l}\mathrm{p} \\
(\mathrm{WT}- \\
\mathrm{M} / \mathrm{WT} \\
\mathrm{C})\end{array}$ & $\begin{array}{l}\mathrm{p} \\
\left(\mathrm{KO}_{-}\right. \\
\mathrm{C} / \mathrm{WT} \\
\left.\mathrm{C}_{-}\right)\end{array}$ & $\begin{array}{l}\mathrm{p} \\
\left(\mathrm{KO}_{-}\right. \\
\mathrm{M} / \mathrm{WT} \\
\left.{ }_{-} \mathrm{M}\right)\end{array}$ & $\begin{array}{l}\text { FDR } \\
\left(\mathrm{KO} \_\mathrm{M} /\right. \\
\text { KO_C) }\end{array}$ & $\begin{array}{l}\text { FDR } \\
(\mathrm{WT} \text {-M/ } \\
\text { WT_C) }\end{array}$ & $\begin{array}{l}\text { FDR } \\
\left(\mathrm{KO} \_\mathrm{C} /\right. \\
\text { WT_C) }\end{array}$ & $\begin{array}{l}\text { FDR } \\
\text { (KO_M/ } \\
\text { WT_M) }\end{array}$ \\
\hline Sequestosome-1 & Sqstm1 & 2.36 & 2.62 & 0.84 & 0.57 & $\begin{array}{r}2.48 \mathrm{E}- \\
05\end{array}$ & $\begin{array}{r}1.88 \mathrm{E}- \\
04\end{array}$ & $\begin{array}{r}1.10 \mathrm{E}- \\
01\end{array}$ & $\begin{array}{r}4.18 \mathrm{E}- \\
03\end{array}$ & + & + & & + \\
\hline Growth/differentiation factor 15 & Gdf15 & 0.77 & 1.52 & 0.08 & -0.67 & $\begin{array}{r}5.97 \mathrm{E}- \\
04\end{array}$ & $\begin{array}{r}7.04 \mathrm{E}- \\
05\end{array}$ & $\begin{array}{r}6.70 \mathrm{E}- \\
01\end{array}$ & $\begin{array}{r}3.02 \mathrm{E}- \\
03\end{array}$ & + & + & & + \\
\hline Metalloproteinase inhibitor 1 & Timp1 & 1.09 & 1.43 & -0.31 & -0.65 & $\mathrm{NaN}$ & $\begin{array}{r}4.17 \mathrm{E}- \\
04\end{array}$ & $\mathrm{NaN}$ & $\begin{array}{r}1.47 \mathrm{E}- \\
02\end{array}$ & & + & & + \\
\hline $\begin{array}{l}\text { Heparanase; Heparanase } 8 \text { kDa } \\
\text { subunit; Heparanase } 50 \text { kDa } \\
\text { subunit }\end{array}$ & Hpse & 0.44 & 1.32 & -0.18 & -1.05 & $\begin{array}{r}5.13 \mathrm{E}- \\
02\end{array}$ & $\begin{array}{r}1.15 \mathrm{E}- \\
05\end{array}$ & $\begin{array}{r}2.51 \mathrm{E}- \\
01\end{array}$ & $\begin{array}{r}3.23 \mathrm{E}- \\
04\end{array}$ & + & + & & + \\
\hline $\begin{array}{l}\text { Plasminogen activator inhibitor } 2 \text {, } \\
\text { macrophage }\end{array}$ & Serpinb2 & 0.16 & 1.21 & -0.26 & -1.30 & $\mathrm{NaN}$ & $\begin{array}{r}2.27 \mathrm{E}- \\
03\end{array}$ & $\mathrm{NaN}$ & $\begin{array}{r}8.42 E- \\
04\end{array}$ & & + & & + \\
\hline
\end{tabular}




\begin{tabular}{|c|c|c|c|c|c|c|c|c|c|c|c|c|c|}
\hline $\begin{array}{l}\text { NADH dehydrogenase } \\
\text { [ubiquinone] iron-sulfur protein } 6 \text {, } \\
\text { mitochondrial }\end{array}$ & Ndufs6 & $\mathrm{NaN}$ & 1.11 & $\mathrm{NaN}$ & 0.85 & $\mathrm{NaN}$ & $\begin{array}{r}8.99 E- \\
03\end{array}$ & $\mathrm{NaN}$ & $\begin{array}{r}2.52 \mathrm{E}- \\
04\end{array}$ & & + & & + \\
\hline Tenascin & Tnc & 0.79 & 1.06 & -0.42 & -0.69 & $\begin{array}{r}5.03 E- \\
04\end{array}$ & $\begin{array}{r}7.22 \mathrm{E}- \\
05\end{array}$ & $\begin{array}{r}4.45 \mathrm{E}- \\
02\end{array}$ & $\begin{array}{r}9.48 \mathrm{E}- \\
05\end{array}$ & + & + & & + \\
\hline Chondroitin sulfate proteoglycan 4 & Cspg4 & 0.61 & 1.03 & -0.85 & -1.27 & $\begin{array}{r}3.57 \mathrm{E}- \\
05\end{array}$ & $\begin{array}{r}1.17 \mathrm{E}- \\
04\end{array}$ & $\begin{array}{r}1.78 \mathrm{E}- \\
04\end{array}$ & $\begin{array}{r}1.70 \mathrm{E}- \\
06\end{array}$ & + & + & + & + \\
\hline Transgelin & Tagln & 0.19 & 0.98 & -1.27 & -2.06 & $\begin{array}{r}2.80 \mathrm{E}- \\
01\end{array}$ & $\begin{array}{r}4.56 \mathrm{E}- \\
04\end{array}$ & $\begin{array}{r}1.93 E- \\
04\end{array}$ & $\begin{array}{r}1.36 \mathrm{E}- \\
06\end{array}$ & & + & + & + \\
\hline Meteorin-like protein & Metrnl & 0.67 & 0.95 & -0.36 & -0.65 & $\begin{array}{r}7.66 \mathrm{E}- \\
06\end{array}$ & $\begin{array}{r}1.48 \mathrm{E}- \\
04\end{array}$ & $\begin{array}{r}1.32 \mathrm{E}- \\
02\end{array}$ & $\begin{array}{r}2.77 \mathrm{E}- \\
04\end{array}$ & + & + & & + \\
\hline $\begin{array}{l}\text { EH domain-binding protein 1-like } \\
\text { protein } 1\end{array}$ & Ehbp1I1 & 1.25 & 0.93 & 0.19 & 0.50 & $\begin{array}{r}1.81 \mathrm{E}- \\
04\end{array}$ & $\begin{array}{r}2.82 \mathrm{E}- \\
05\end{array}$ & $\begin{array}{r}3.08 \mathrm{E}- \\
01\end{array}$ & $\begin{array}{r}6.16 \mathrm{E}- \\
03\end{array}$ & + & + & & + \\
\hline $\begin{array}{l}\text { ATP synthase subunit beta, } \\
\text { mitochondrial }\end{array}$ & Atp5b & 1.44 & 0.81 & 0.16 & 0.78 & $\begin{array}{r}5.03 E- \\
06\end{array}$ & $\begin{array}{r}1.68 \mathrm{E}- \\
03\end{array}$ & $\begin{array}{r}2.46 \mathrm{E}- \\
01\end{array}$ & $\begin{array}{r}2.66 \mathrm{E}- \\
03\end{array}$ & + & + & & + \\
\hline $\begin{array}{l}\text { Pro-neuropeptide Y;Neuropeptide } \\
\text { Y;C-flanking peptide of NPY }\end{array}$ & Npy & 0.64 & 0.81 & -0.80 & -0.97 & $\mathrm{NaN}$ & $\begin{array}{r}9.54 \mathrm{E}- \\
03\end{array}$ & $\mathrm{NaN}$ & $\begin{array}{r}1.05 \mathrm{E}- \\
03\end{array}$ & & + & & + \\
\hline $\begin{array}{l}\text { Insulin-like growth factor-binding } \\
\text { protein } 7\end{array}$ & $\operatorname{lgfbp} 7$ & 0.31 & 0.80 & -0.28 & -0.77 & $\begin{array}{r}6.07 \mathrm{E}- \\
02\end{array}$ & $\begin{array}{r}1.76 \mathrm{E}- \\
03\end{array}$ & $\begin{array}{r}1.58 \mathrm{E}- \\
01\end{array}$ & $\begin{array}{r}5.38 \mathrm{E}- \\
04\end{array}$ & & + & & + \\
\hline Angiopoietin-2 & Angpt2 & 0.19 & 0.79 & -0.87 & -1.48 & $\begin{array}{r}5.04 \mathrm{E}- \\
01\end{array}$ & $\begin{array}{r}1.72 \mathrm{E}- \\
02\end{array}$ & $\begin{array}{r}1.05 \mathrm{E}- \\
02\end{array}$ & $\begin{array}{r}5.70 \mathrm{E}- \\
04\end{array}$ & & + & + & + \\
\hline Thrombospondin-1 & Thbs 1 & 0.44 & 0.79 & -0.78 & -1.14 & $\begin{array}{r}3.68 \mathrm{E}- \\
04\end{array}$ & $\begin{array}{r}2.86 \mathrm{E}- \\
03\end{array}$ & $\begin{array}{r}2.32 \mathrm{E}- \\
03\end{array}$ & $\begin{array}{r}1.82 \mathrm{E}- \\
06\end{array}$ & + & + & + & + \\
\hline
\end{tabular}




\begin{tabular}{|c|c|c|c|c|c|c|c|c|c|c|c|c|c|}
\hline Fibulin-2 & Fbln2 & 0.31 & 0.79 & -0.45 & -0.92 & $\begin{array}{r}4.89 \mathrm{E}- \\
02\end{array}$ & $\begin{array}{r}1.10 \mathrm{E}- \\
02\end{array}$ & $\begin{array}{r}1.27 \mathrm{E}- \\
01\end{array}$ & $\begin{array}{r}3.21 \mathrm{E}- \\
06\end{array}$ & & + & & + \\
\hline Endothelial protein $\mathrm{C}$ receptor & Procr & 0.34 & 0.76 & -0.08 & -0.50 & $\begin{array}{r}8.79 E- \\
03\end{array}$ & $\begin{array}{r}7.07 \mathrm{E}- \\
03\end{array}$ & $\begin{array}{r}6.18 \mathrm{E}- \\
01\end{array}$ & $\begin{array}{r}2.82 \mathrm{E}- \\
02\end{array}$ & + & + & & + \\
\hline T-lymphocyte surface antigen Ly-9 & Ly9 & 0.25 & 0.73 & -0.07 & -0.55 & $\begin{array}{r}4.56 \mathrm{E}- \\
02\end{array}$ & $\begin{array}{r}2.51 \mathrm{E}- \\
04\end{array}$ & $\begin{array}{r}3.69 \mathrm{E}- \\
01\end{array}$ & $\begin{array}{r}4.70 \mathrm{E}- \\
03\end{array}$ & & + & & + \\
\hline Plasminogen activator inhibitor 1 & Serpine1 & 0.43 & 0.72 & -1.23 & -1.51 & $\begin{array}{r}7.76 \mathrm{E}- \\
02\end{array}$ & $\begin{array}{r}8.89 E- \\
03\end{array}$ & $\begin{array}{r}3.34 \mathrm{E}- \\
04\end{array}$ & $\begin{array}{r}1.17 \mathrm{E}- \\
04\end{array}$ & & + & + & + \\
\hline Lactadherin & Mfge8 & 0.21 & 0.71 & -0.32 & -0.82 & $\begin{array}{r}1.88 \mathrm{E}- \\
02\end{array}$ & $\begin{array}{r}3.03 E- \\
04\end{array}$ & $\begin{array}{r}8.04 \mathrm{E}- \\
04\end{array}$ & $\begin{array}{r}1.49 \mathrm{E}- \\
04\end{array}$ & & + & & + \\
\hline $\begin{array}{l}\text { Hepatocyte growth factor; } \\
\text { Hepatocyte growth factor alpha } \\
\text { chain; Hepatocyte growth factor } \\
\text { beta chain }\end{array}$ & $\mathrm{Hgf}$ & 0.31 & 0.69 & -0.23 & -0.61 & $\begin{array}{r}3.48 \mathrm{E}- \\
02\end{array}$ & $\begin{array}{r}3.40 \mathrm{E}- \\
04\end{array}$ & $\begin{array}{r}8.30 \mathrm{E}- \\
02\end{array}$ & $\begin{array}{r}9.89 \mathrm{E}- \\
04\end{array}$ & & + & & + \\
\hline Peroxidasin homolog & Pxdn & 0.18 & 0.67 & -0.47 & -0.96 & $\begin{array}{r}4.59 \mathrm{E}- \\
01\end{array}$ & $\begin{array}{r}2.99 \mathrm{E}- \\
02\end{array}$ & $\begin{array}{r}1.03 \mathrm{E}- \\
01\end{array}$ & $\begin{array}{r}2.81 \mathrm{E}- \\
03\end{array}$ & & + & & + \\
\hline $\begin{array}{l}\text { NAD-dependent protein } \\
\text { deacetylase sirtuin- } 2\end{array}$ & Sirt2 & 1.75 & 0.59 & -0.02 & 1.14 & $\begin{array}{r}5.59 E- \\
09\end{array}$ & $\begin{array}{r}1.40 \mathrm{E}- \\
03\end{array}$ & $\begin{array}{r}8.56 \mathrm{E}- \\
01\end{array}$ & $\begin{array}{r}3.31 \mathrm{E}- \\
07\end{array}$ & + & + & & + \\
\hline Collectin-12 & Colec12 & 0.41 & 0.56 & -0.91 & -1.06 & $\begin{array}{r}1.33 \mathrm{E}- \\
03\end{array}$ & $\begin{array}{r}2.59 \mathrm{E}- \\
03\end{array}$ & $\begin{array}{r}8.05 \mathrm{E}- \\
06\end{array}$ & $\begin{array}{r}3.06 \mathrm{E}- \\
05\end{array}$ & + & + & + & + \\
\hline Laminin subunit beta-1 & Lamb1 & -0.02 & 0.56 & -0.11 & -0.69 & $\begin{array}{r}8.95 \mathrm{E}- \\
01\end{array}$ & $\begin{array}{r}4.97 \mathrm{E}- \\
02\end{array}$ & $\begin{array}{r}6.47 \mathrm{E}- \\
01\end{array}$ & $\begin{array}{r}5.80 \mathrm{E}- \\
03\end{array}$ & & + & & + \\
\hline
\end{tabular}




\begin{tabular}{|c|c|c|c|c|c|c|c|c|c|c|c|c|c|}
\hline $\begin{array}{l}\text { Non-histone chromosomal protein } \\
\text { HMG-17 }\end{array}$ & Hmgn2 & -0.85 & 0.54 & 0.63 & -0.76 & $\begin{array}{r}2.83 \mathrm{E}- \\
04\end{array}$ & $\begin{array}{r}2.24 \mathrm{E}- \\
03\end{array}$ & $\begin{array}{r}1.88 \mathrm{E}- \\
03\end{array}$ & $\begin{array}{r}2.61 \mathrm{E}- \\
04\end{array}$ & + & + & + & + \\
\hline Protein CTLA-2-alpha & Ctla2a & -0.42 & 0.53 & -0.03 & -0.98 & $\begin{array}{r}1.60 \mathrm{E}- \\
01\end{array}$ & $\begin{array}{r}1.65 \mathrm{E}- \\
02\end{array}$ & $\begin{array}{r}9.25 \mathrm{E}- \\
01\end{array}$ & $\begin{array}{r}1.75 \mathrm{E}- \\
05\end{array}$ & & + & & + \\
\hline $\begin{array}{l}\text { Calcium/calmodulin-dependent } \\
\text { protein kinase type II subunit delta }\end{array}$ & Camk2d & 1.18 & 0.53 & 0.15 & 0.80 & $\begin{array}{r}3.01 \mathrm{E}- \\
05\end{array}$ & $\begin{array}{r}1.64 \mathrm{E}- \\
02\end{array}$ & $\begin{array}{r}4.24 \mathrm{E}- \\
01\end{array}$ & $\begin{array}{r}4.44 \mathrm{E}- \\
04\end{array}$ & + & + & & + \\
\hline Stromelysin-1 & Mmp3 & 0.65 & 0.53 & 1.07 & 1.20 & $\begin{array}{r}1.35 \mathrm{E}- \\
02\end{array}$ & $\begin{array}{r}3.47 \mathrm{E}- \\
02\end{array}$ & $\begin{array}{r}1.03 E- \\
03\end{array}$ & $\begin{array}{r}3.36 \mathrm{E}- \\
04\end{array}$ & + & + & + & + \\
\hline $45 \mathrm{kDa}$ calcium-binding protein & Sdf4 & 0.26 & 0.51 & -0.38 & -0.62 & $\begin{array}{r}5.79 \mathrm{E}- \\
04\end{array}$ & $\begin{array}{r}1.56 \mathrm{E}- \\
03\end{array}$ & $\begin{array}{r}1.71 \mathrm{E}- \\
04\end{array}$ & $\begin{array}{r}3.08 \mathrm{E}- \\
04\end{array}$ & + & + & + & + \\
\hline Annexin A5 & Anxa5 & 0.41 & -0.53 & -0.17 & 0.76 & $\begin{array}{r}3.77 \mathrm{E}- \\
02\end{array}$ & $\begin{array}{r}3.14 \mathrm{E}- \\
02\end{array}$ & $\begin{array}{r}3.52 \mathrm{E}- \\
01\end{array}$ & $\begin{array}{r}4.27 \mathrm{E}- \\
03\end{array}$ & + & + & & + \\
\hline $\begin{array}{l}\text { SLIT-ROBO Rho GTPase- } \\
\text { activating protein } 2\end{array}$ & Srgap2 & -0.07 & -0.60 & 0.03 & 0.56 & $\begin{array}{r}6.45 \mathrm{E}- \\
01\end{array}$ & $\begin{array}{r}1.52 \mathrm{E}- \\
02\end{array}$ & $\begin{array}{r}8.31 \mathrm{E}- \\
01\end{array}$ & $\begin{array}{r}2.12 \mathrm{E}- \\
02\end{array}$ & & + & & + \\
\hline $\begin{array}{l}\text { Nuclease-sensitive element- } \\
\text { binding protein } 1\end{array}$ & Ybx1 & -1.00 & -0.63 & 1.12 & 0.75 & $\begin{array}{r}1.12 \mathrm{E}- \\
03\end{array}$ & $\begin{array}{r}1.05 E- \\
02\end{array}$ & $\begin{array}{r}4.68 \mathrm{E}- \\
04\end{array}$ & $\begin{array}{r}4.69 E- \\
03\end{array}$ & + & + & + & + \\
\hline Rabankyrin-5 & Ankfy1 & -0.44 & -0.65 & 0.35 & 0.56 & $\begin{array}{r}1.75 \mathrm{E}- \\
03\end{array}$ & $\begin{array}{r}6.72 \mathrm{E}- \\
03\end{array}$ & $\begin{array}{r}6.62 \mathrm{E}- \\
03\end{array}$ & $\begin{array}{r}1.32 \mathrm{E}- \\
02\end{array}$ & + & + & & + \\
\hline $\begin{array}{l}\text { Lysosomal acid lipase/cholesteryl } \\
\text { ester hydrolase }\end{array}$ & Lipa & -1.09 & -1.11 & -0.52 & -0.50 & $\begin{array}{r}7.96 \mathrm{E}- \\
06\end{array}$ & $\begin{array}{r}6.06 \mathrm{E}- \\
04\end{array}$ & $\begin{array}{r}2.43 \mathrm{E}- \\
02\end{array}$ & $\begin{array}{r}5.55 \mathrm{E}- \\
03\end{array}$ & + & + & & + \\
\hline $\begin{array}{l}\text { Very-long-chain 3-oxoacyl-CoA } \\
\text { reductase }\end{array}$ & Hsd17b12 & 0.55 & -1.22 & -0.90 & 0.86 & $\begin{array}{r}1.18 \mathrm{E}- \\
01\end{array}$ & $\begin{array}{r}4.14 \mathrm{E}- \\
04\end{array}$ & $\begin{array}{r}2.42 \mathrm{E}- \\
02\end{array}$ & $\begin{array}{r}2.66 \mathrm{E}- \\
03\end{array}$ & & + & + & + \\
\hline
\end{tabular}




\begin{tabular}{|l|l|r|r|r|r|r|r|r|r|l|l|l|}
\hline Beta-galactosidase & Glb1 & -1.44 & -1.42 & -0.52 & -0.54 & 07 & 07 & 04 & 04 & + & + \\
\hline Acylamino-acid-releasing enzyme & Apeh & -1.43 & -1.49 & 0.46 & 0.52 & 06 & 04 & 02 & 02 & + & + \\
\hline
\end{tabular}


Appendix 2. Pathway analysis of the proteomic data of the cell lysate of cultured microglia using the gene ontology enrichment analysis and visualization tool (GOrilla). (A) WT microglia after the exposure to myelin compared with control. (B) Myd88 ${ }^{-/}$microglia compared with WT microglia after the exposure to myelin debris. (C) Myd88 ${ }^{-/}$microglia after the exposure to myelin compared with control.

\section{(A) Myelin_WT/control_WT: overview}

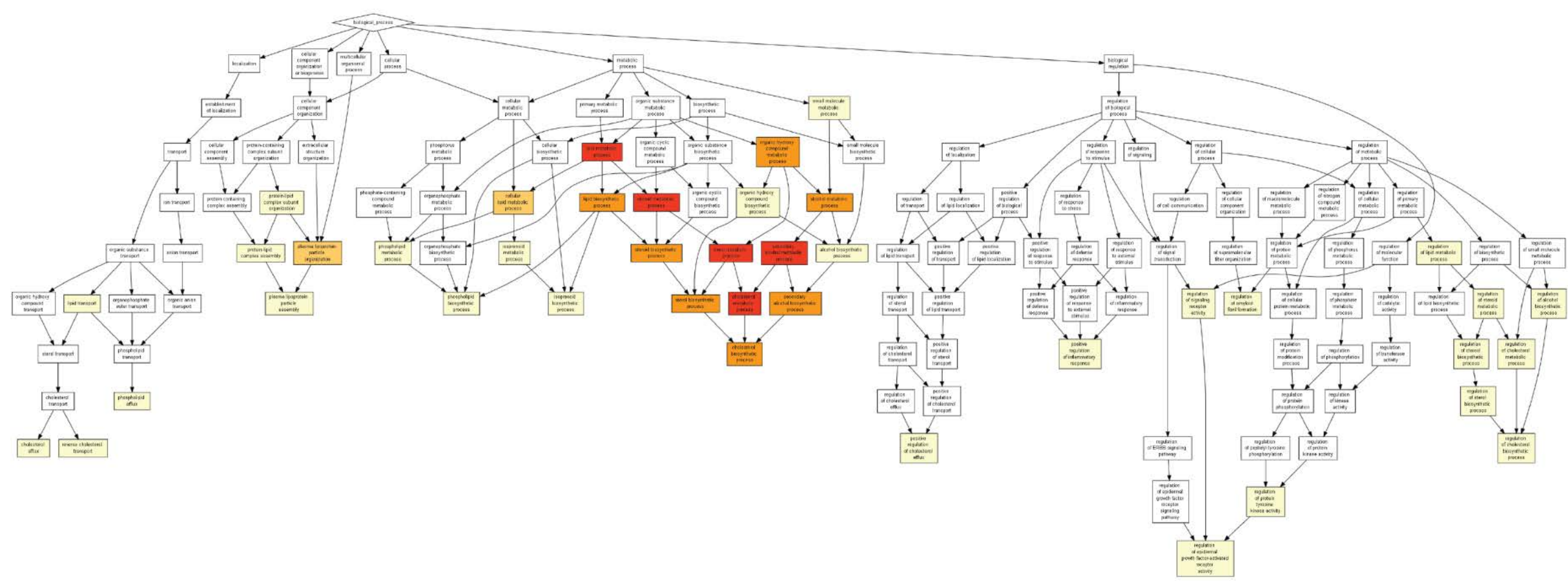


(A) Myelin_WT/control_WT: lipid metabolic processes

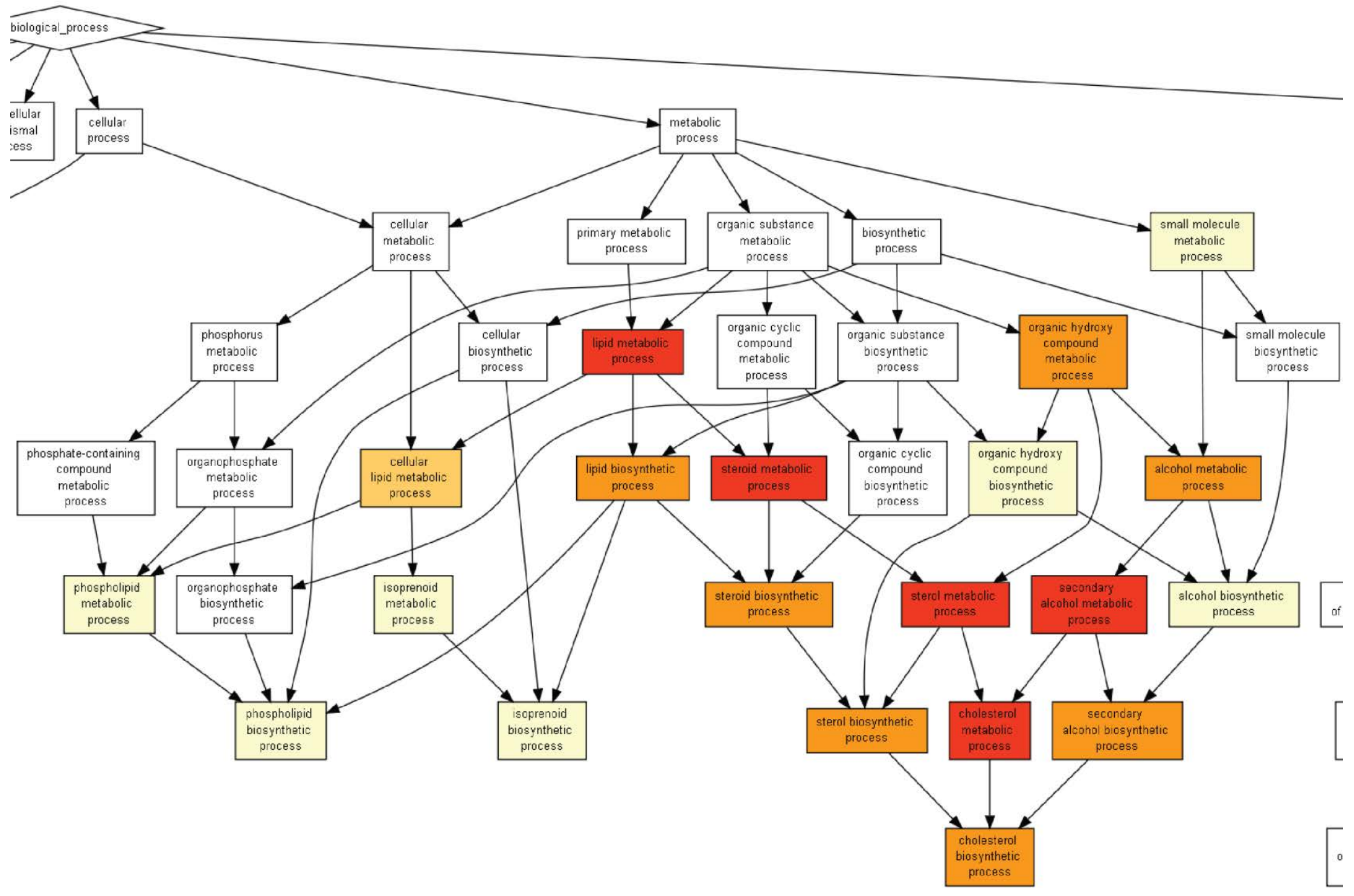


(A) Myelin_WT/control_WT: lipid transport

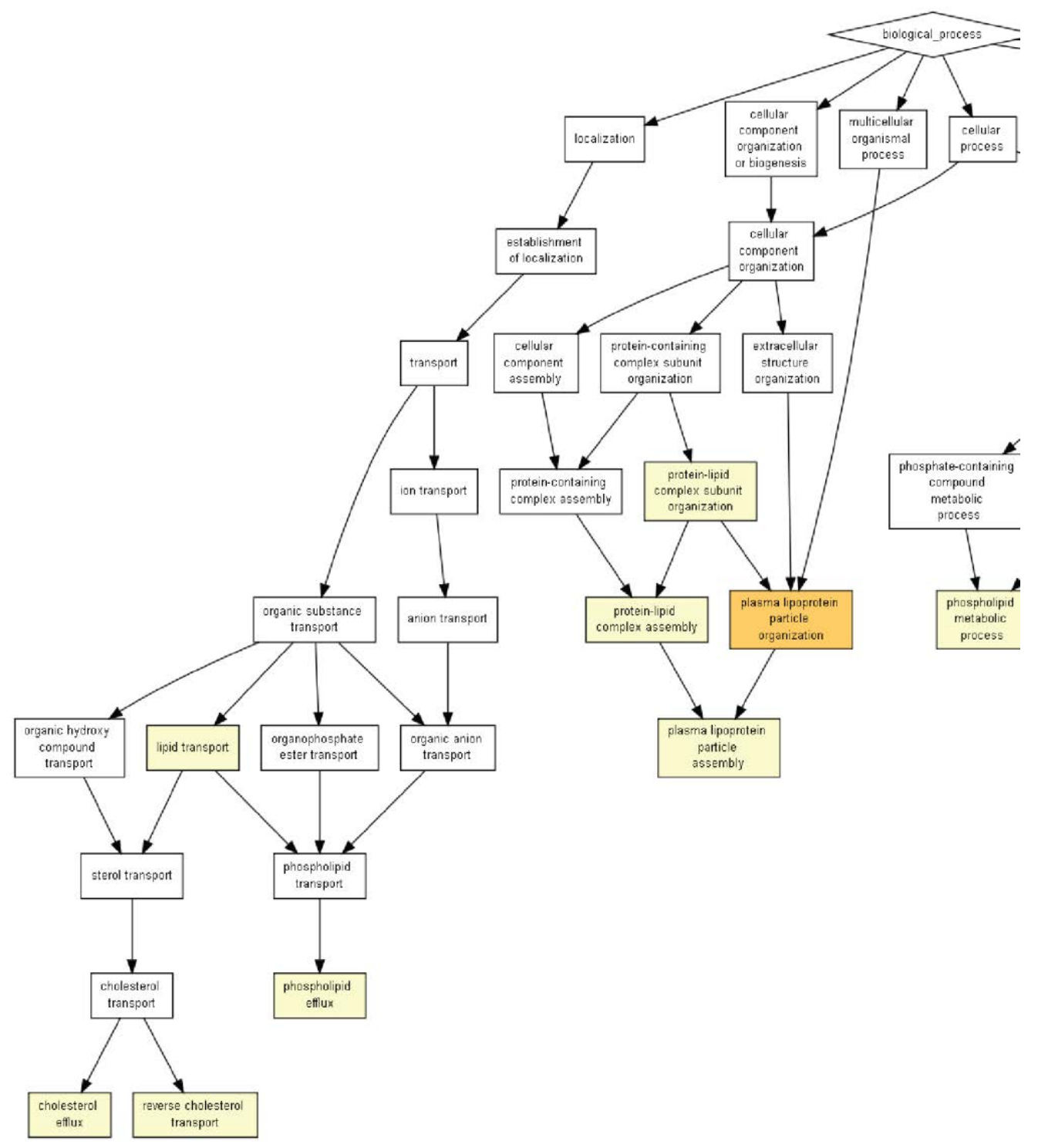


(B) Myelin_Myd88--/myelin_WT

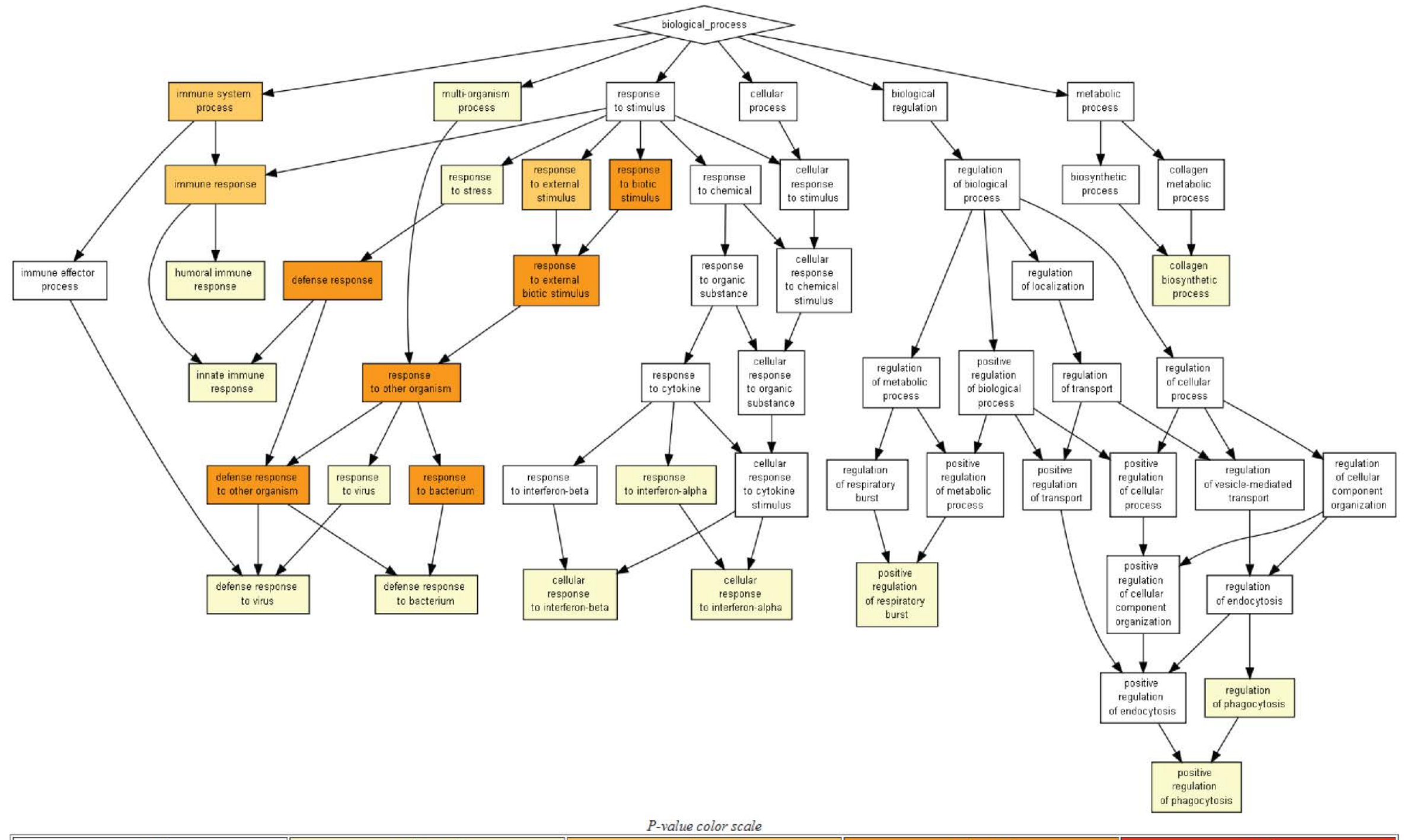




\section{(C) Myelin_Myd88-/control_Myd88-/-: overview}

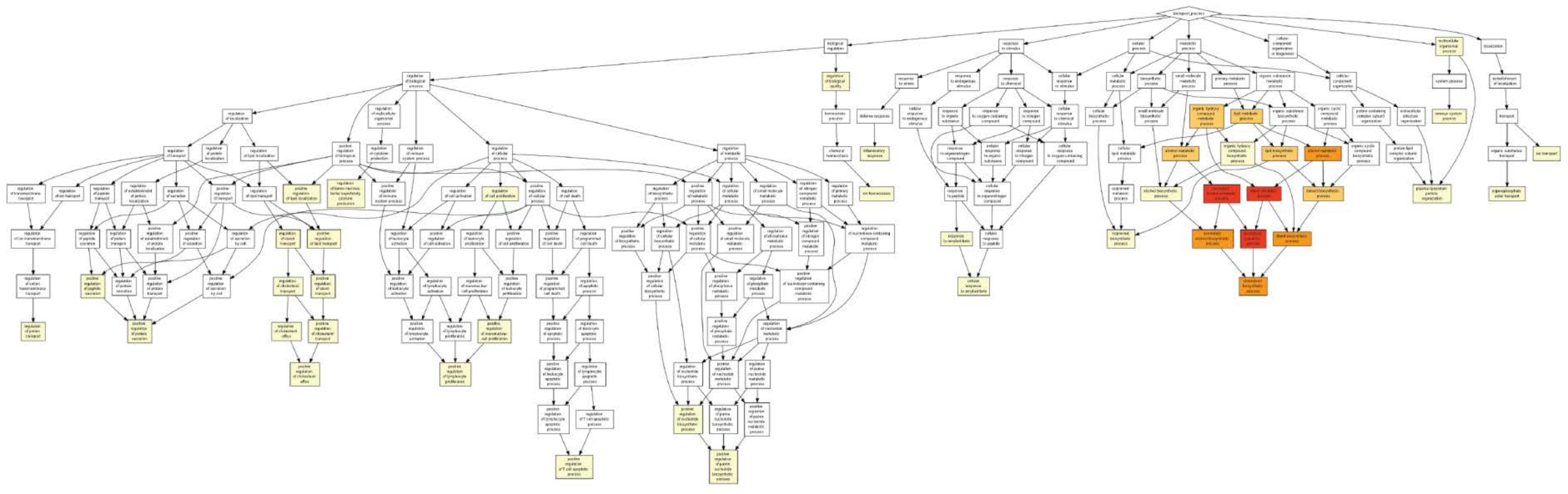


(C) Myelin_Myd88\%/control_Myd88 $\%$ : lipid metabolic processes

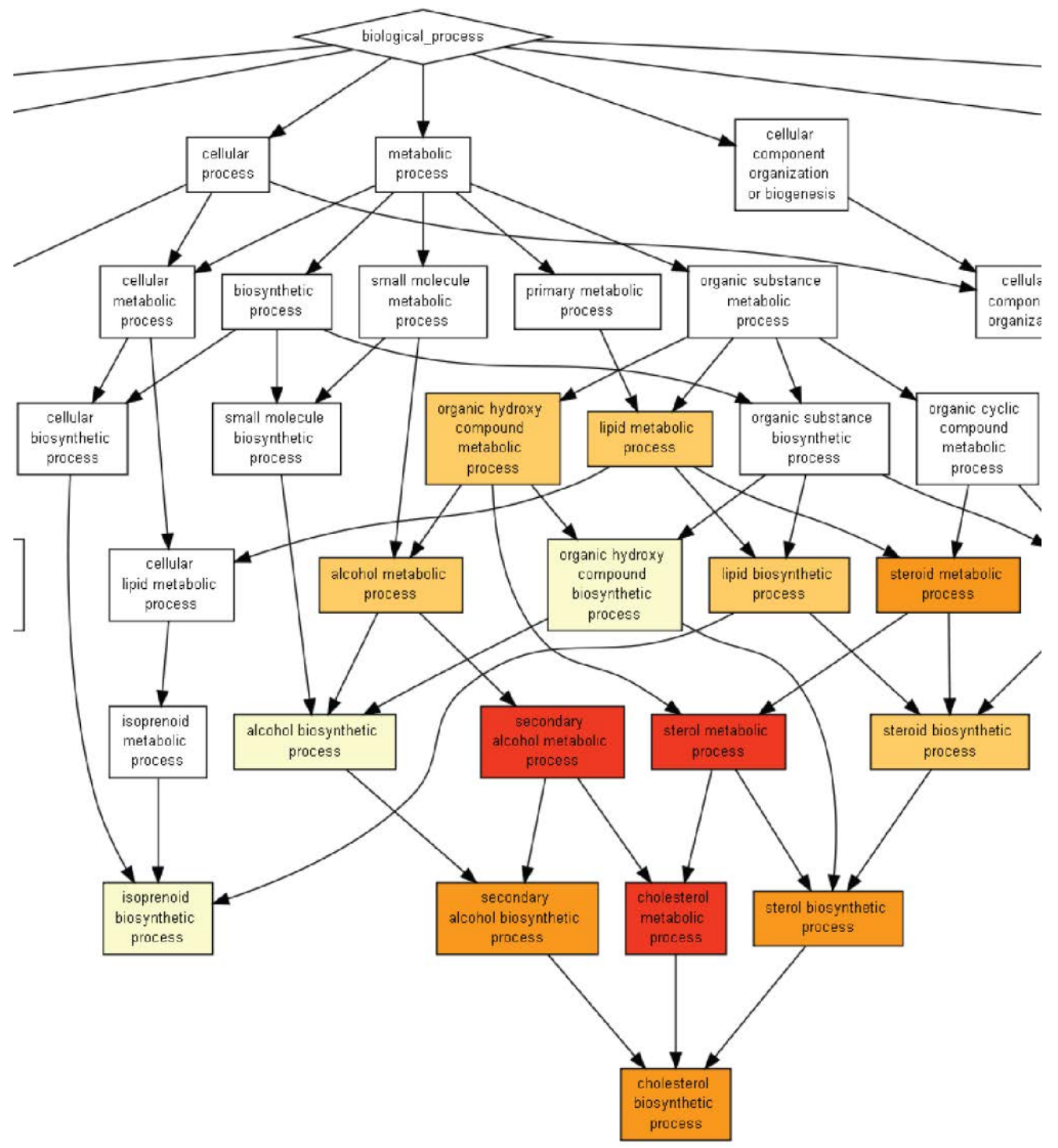




\section{List of abbreviations}

ABCA1: ATP-binding cassette sub-family A member 1

AIF1: allograft inflammatory factor 1 (also known as IBA1)

AFN: atipamezole, flumazenil and naloxone

AP-1: activator protein 1

APC (CC-1): Adenomatous polyposis coli protein (antibody clone CC-1)

v-ATPase: vacuolar-type $\mathrm{H}^{+}$-ATPases

BCAS1: breast carcinoma-amplified sequence 1 homolog

BMDM: bone marrow-derived macrophages

BME: Basal Medium Eagle

bp: base pair

BSA: bovine serum albumin

CC: corpus callosum

CHD: chromodomain helicase DNA-binding

$\mathrm{Cl}$ : confidence interval

CL-12: collectin-12

CNP: 2',3'-Cyclic nucleotide 3'-phosphodiesterase

CNS: central nervous system

CSF1: also known as M-CSF

CT-B: cholera toxin subunit B

CX3CR1: CX3C chemokine receptor 1

DAMP: damage-associated molecular pattern

DAPI: 4',6-diamidino-2-phenylindole

DIV: days in vitro

DMEM: Dulbecco's Modified Eagle Medium

DPI: days post-injection

DPT: days post-treatment

EAE: experimental autoimmune encephalomyelitis

EDTA: ethylenediaminetetraacetic acid

EdU: 5-ethynyl-2'-deoxyuridine

EM: electron microscopy 
ERK: extracellular signal-regulated kinase

FACS: fluorescence-activated cell sorting

FDR: false discovery rate

Fiji: Fiji is just ImageJ

FPKM: fragments per kilobase of transcript per million mapped reads

Galectin-3: galactose-specific lectin 3 (formally called Mac-2 antigen)

GDF-15: growth/differentiation factor 15

GFAP: glial fibrillary acidic protein

GOrilla: gene ontology enrichment analysis and visualization tool

h: hour(s)

HBSS: Hank's Balanced Salt Solution

HEPES: 4-(2-hydroxyethyl)-1-piperazineethanesulfonic acid)

HS: horse serum

IB: immunoblotting

IBA1: ionized calcium binding adaptor molecule 1 (also known as AIF1)

ICC: immunocytochemistry

IFN: interferon

IGF: insulin-like growth factor

IHC: immunohistochemistry

IL: interleukin

iNOS: inducible nitric oxide synthase

IP: intraperitoneal(ly)

IPA: Ingenuity Pathway Analysis

JAK: Janus kinase

KO: knockout

LAMP-2: lysosome-associated membrane glycoprotein 2 (CD107b or Mac-3)

LFB-PAS: luxol fast blue and periodic acid-Schiff

LFQ: label-free quantification

LINGO1: leucine-rich repeat and immunoglobulin-like domain-containing nogo receptorinteracting protein 1

LPS: lipopolysaccharide

MACS: magnetic-activated cell sorting 
MAP: mitogen-activated protein kinase

MBP: myelin basic protein

M-CSF: macrophage colony-stimulating factor (also known as CSF1)

$\beta-M E:$ 2-mercaptoethanol

MHC-II: major histocompatibility complex class II

min: minute(s)

MMF: medetomidine, midazolam and fentanyl

MOG: myelin oligodendrocyte glycoprotein

MS: multiple sclerosis

MyD88: myeloid differentiation primary response protein MyD88

NaN: not a number

NF-H: neurofilament heavy polypeptide

NF-kB: nuclear factor-kB

NG2: neural/glial antigen 2; homolog proteins include mouse AN2 and human chondroitin sulfate proteoglycan 4 (CSPG4), etc.

NKX-2.2: homeobox protein NKX-2.2

OCSC: organotypic cerebellar slice culture

OHSC: organotypic hippocampal slice culture

OLIG2: oligodendrocyte transcription factor 2

OPC: oligodendrocyte progenitor/precursor cell

P: post-natal day

PAGE: polyacrylamide gel electrophoresis

PAMP: pathogen-associated molecular pattern

PBS: phosphate buffered saline

PCR: polymerase chain reaction

PDGFRa: platelet-derived growth factor receptor alpha

PFA: paraformaldehyde

PLL: poly-L-lysine

PLP: proteolipid protein

PMSF: phenylmethylsulfonyl fluoride

PNS: peripheral nervous system

PPAR: peroxisome proliferator-activated receptor 
PRR: pattern recognition receptor

RNA-seq: RNA sequencing

ROI: region of interest

$\mathrm{RT}$ : room temperature

RT-PCR: reverse transcription polymerase chain reaction

RXR: retinoid X receptor

SALL1: spalt-like transcription factor 1

SC: subcutaneous(ly)

SD: standard deviation

sec: second(s)

SOAT1: sterol O-acyltransferase 1

STAT: signal transducer and activator of transcription

SVZ: subventricular zone

T3: 3,3',5-triiodo-L-thyronine

TAE: Tris-acetate-EDTA

TGF: transforming growth factor

TIMP-1: metalloproteinase inhibitor 1; also known as tissue inhibitor of metalloproteinase 1

TLR: toll-like receptor

TMEM119: transmembrane protein 119

TNF: tumor necrosis factor

TRIF: TIR (Toll IL-1R) domain-containing adaptor-inducing interferon- $\beta$

UV: ultraviolet

WT: wild-type 


\section{List of figures}

Figure 1. Remyelination was defective in the spinal cord of $M y d 88^{-/}$mice

Figure 2. Microglia / macrophages containing myelin lipids remained in the lesions of Myd88 $/$ mice at $14 \mathrm{DPI}$. (A) and (B) IBA $1^{+}$microglia / macrophages (magenta) were recruited to the lesions of both WT and $M y d 88^{-/}$mice at $7 \mathrm{DPI}$, and the density of IBA $1^{+}$ cells decreased afterwards. At 14 DPI (A), the proportion of the volume occupied by IBA $1^{+}$ cells in the lesions (devoid of FluoroMyelin Green stain) was higher in Myd88- than WT. Welch's $t$-test. (Error bars: $95 \% \mathrm{Cl} ; n=4-9$ lesions) (C) More myelin lipids accumulated in microglia / macrophages in the lesions of $M y d 88^{-/}$mice at $14 \mathrm{DPI}$, analyzed by the proportion of the volume occupied by both FluoroMyelin Green stain (green) and IBA1 (magenta) (co-localization, white) in the lesions. Welch's $t$-test. (Error bars: 95\% Cl; $n=4$ or 5 lesions) (D) The amount of myelin basic protein (MBP) inside IBA $1^{+}$cells was similar in the lesions of Myd88 ${ }^{-1}$ and WT mice.

Figure 3. Astrocytes surrounded demyelinated lesions at $7 \mathrm{DPI}$ and were in the lesions at $21 \mathrm{DPI}$ .45

Figure 4. Cultured microglia internalized more myelin debris than non-myelin membrane.

Figure 5. The amount of PKH67-labeled myelin in cultured microglia measured by the integrated intensity of PKH67 in each cell.

Figure 6. Less fusion of phagosomes containing myelin debris with (endo)lysosomes was observed in cultured $M y d 88^{--}$than WT microglia 60 min after the treatment with myelin debris.

Figure 7. LAMP-2 proteins were assembled in the membrane of endolysosomes containing myelin debris in cultured microglia.

Figure 8. Volcano plots show the relative amount of proteins in the supernatant of microglial cell cultures.

Figure 9. Method to identify the differentially expressed genes from two comparisons. ..55

Figure 10. Differential gene expression analyses of the proteomic data of microglial cell lysate.

Figure 11. IFN-y was predicted by Ingenuity Pathway Analysis to be an upstream regulator

Figure 12. Differential gene expression analysis of the transcriptomes of demyelinated lesions in Myd88 ${ }^{-1}$ compared with WT mice.

Figure 13. Myelination in organotypic hippocampal slice culture (OHSC). .64 
Figure 14. Lysolecithin-induced focal demyelination in OHSC......

Figure 15. MOG antibody and complement induced demyelination in WT OHSC. .66

Figure 16. The recruitment of OPCs to developing white matter in the corpus callosum (CC) of $M y d 88^{-/}$mice was not affected. .68 


\section{List of tables}

Table 1. The media for the cultures of primary microglia, mixed glial culture, bone marrowderived macrophages and L929 cell line, treatment, and live cell imaging. 24

Table 2. Seeding of microglial cell cultures.

Table 3. Components of the OHSC culture medium and the Medium A for OHSC preparation.

Table 4. Buffers and solutions for immunohistochemistry and immunocytochemistry...... 33

Table 5. Primary antibodies used in IHC.

Table 6. Secondary antibodies used in IHC

Table 7. Primary antibodies used in ICC.

Table 8. Secondary antibodies and dyes used in ICC

Table 9. Differentially expressed genes identified to be significant in the proteomic analysis of microglial cell lysate in both comparisons myelin_Myd88 myelin_WT / control WT $\left(\log _{2}(\right.$ expression ratio $)<-0.5$ or $>0.5$, and $p$-value $\left.<0.05\right)$.

Table 10. Selected markers for microglia activation and lysosomal function identified in the proteomic analysis of microglial cell lysate.

Table 11. Selected upstream regulators predicted in both comparisons myelin_Myd88 ${ }^{-1-}$ /myelin_WT and myelin_WT/control WT by Ingenuity Pathway Analysis of the proteome of microglial cell lysate. .59 


\section{Declaration of authorship}

Herewith I declare, that I prepared the PhD thesis "Microglia activation and regulation of remyelination in the central nervous system" on my own and with no other sources and aids than quoted.

Göttingen, 30 ${ }^{\text {th }}$ September 2018

Minhui Su 